\title{
Quantitative PCR Analysis of the Bartonella henselae carD Gene during Bacterial Stress
}

\section{Jacob Simmonds}

\author{
A thesis \\ submitted to the Victoria University of Wellington \\ in fulfilment for the degree of \\ Master of Science
}

Victoria University of Wellington

Te Whare Wānanga o te Ūpoko o te Ika a Māui

2016 



\begin{abstract}
The Bartonella genus is comprised of arthropod-borne, intracellular bacterial pathogens that colonise the mammalian bloodstream. A large number of mammalian species are hosts for one or more Bartonella species, as either reservoir or incidental hosts. Bartonella species are only able to invade and replicate in host red blood cells in the reservoir host, and to be taken up by an associated haematophagous arthropod vector to complete transmission and the bacterial life cycle. Humans are the reservoir hosts for B. quintana and B. bacilliformis, and are incidental hosts for more than 16 additional zoonotic Bartonella species, including $B$. henselae, which is normally carried by cats. B. henselae infection, usually acquired through cat scratches or bites, can result in several clinical manifestations, with varying degrees of severity; the most common of these is cat scratch disease, where symptoms commonly range from enlarged lymph nodes to severe fever. Although usually a mild illness, $B$. henselae infection can occasionally lead to severe symptoms, affecting neurological and other major organ systems.
\end{abstract}

During their life cycle the Bartonellae must adapt to various toxic host environments; such adaptation is mediated by several bacterial stress pathways, which modify bacterial transcription. However, many gaps remain in the understanding of $B$. henselae stress response pathways. The object of this study, the $\operatorname{carD}$ gene, was identified as a possible component of the Bartonella stress response. The $\operatorname{carD}$ gene has been shown to be critical for stress defence in other bacterial species, including Mycobacterium tuberculosis, Thermus thermophilus, and Myxococcus xanthus.

Our study aimed to investigate whether carD played as significant a role during the $B$. henselae response to stresses as it does in other bacterial genera. We first attempted to perform growth comparisons between a $B$. henselae carD mutant strain and a wild type strain during exposure to stress conditions; however, our mutagenic carD plasmid interfered with bacterial growth of Escherichia coli cultures, which hindered transformation and generation of a $B$. henselae carD mutant. As an alternative, we investigated the expression of $B$. henselae carD under stress conditions, comparing $c a r D$ expression during stress against a non-stressed $B$. henselae control, using quantitative PCR. We found no significant difference of expression of the $c a r D$ gene between the control and any of our conditions, although a trend of increased $\operatorname{carD}$ expression was found in several stress conditions. We believe that these findings merit further study into the role of $c a r D$ in the $B$. henselae stress response. 


\section{Acknowledgments}

First and foremost, I would like to thank my supervisor, Dr Joanna MacKichan, without whom this would not be possible. Thank you for all the support you have given me over the last two years and a half, for helping me in the lab with your advice and experience, and for playing a massive role in editing this thesis.

I would also like to thank Dr David Ackerley, Dr Jeremy Owen (pointing me to the Mycobacterial CarD paper, it proved immensely helpful), and to Dr Jane Koehler (for advice, providing Bartonella strains, and plasmids). Thanks to all the members of both the Ackerley and Day Lab for the advice and materials (and top notch food at lab meetings); to Jack and Matt S (for bacterial strains and plasmids), to Alistair (qPCR advice), to Jen and Euan (for assistance and materials), and to especially Varun, who took time out of writing his doctoral thesis, to help not only with my initial qPCR but then following contamination issues. Thanks to all the members of Alan Macdiarmid, for any help and companionship, notably to Matt $\mathrm{R}$ and Dana (for help with qPCR and materials). I would like to thank both the members of the MacKichan lab who have been awesome lab mates; Raphael, Gabby, Alvey, Callum, Haydn, Kurt, and especially Vaughan. Thank you also the occupants of KK702 (and honorary members) for keeping me sane when times got tough, and for distracting me from my masters when needed it; Sonja, James, Vaughan, Kyle, Jasmine (J3), Jack, Jen, Melz, Euan, Izzie, Rory, Eddie, and Mitch.

Thank you to all my friends and family who have supported me not only through undergraduate, but also through my studies. Thanks to my sister Harnah and to Dad. Thanks to Mcfly, Rachel, Rowan, Sam, and Nguyen for keeping me heartily entertained. Lastly, thank you to Jasmine (J2), for waiting patiently for me to finish this thesis. 


\section{Table of Contents}

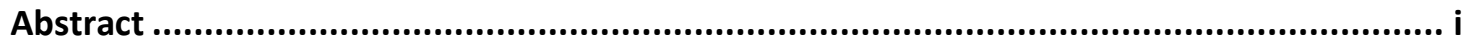

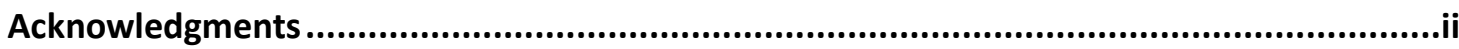

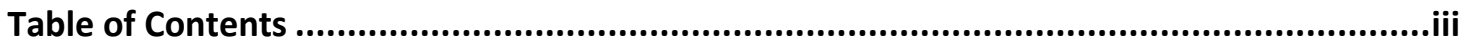

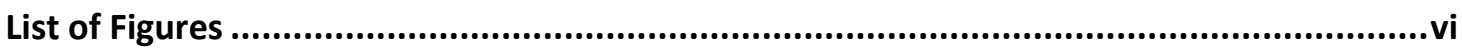

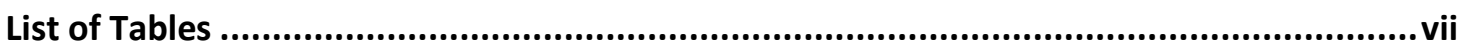

Abbreviations used in this thesis.......................................................................................

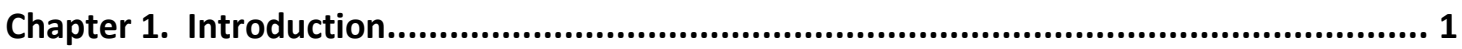

1.1. Human-Specific Bartonella Species.................................................................. 3

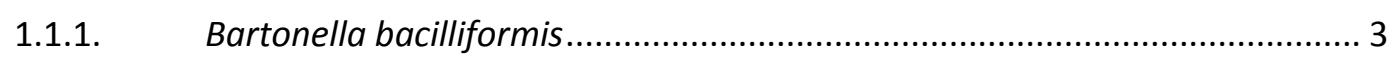

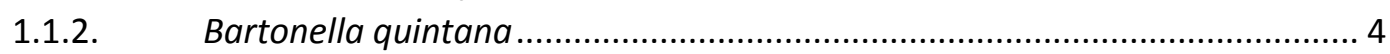

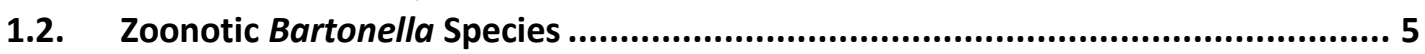

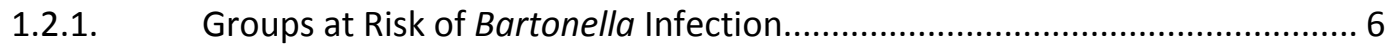

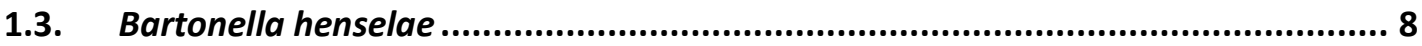

1.3.1. History of $B$. henselae and Cat Scratch Disease............................................. 8

1.3.2. Bartonella henselae, Felis catus, and Ctenocephalides felis.......................... 9

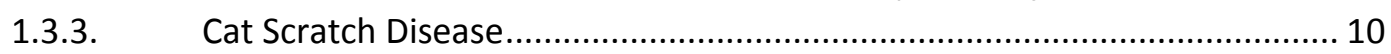

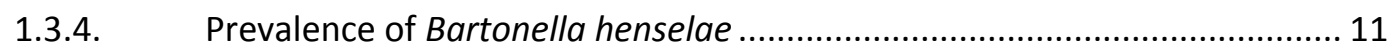

1.4. Pathology and Host Cell Interactions of Bartonella ...........................................14

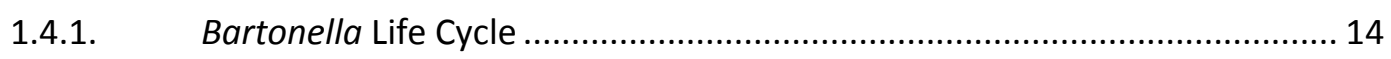

1.4.2. Pathology and Mechanism of Infection of $B$. henselae ................................ 16

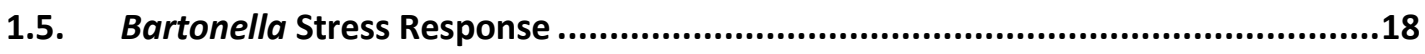

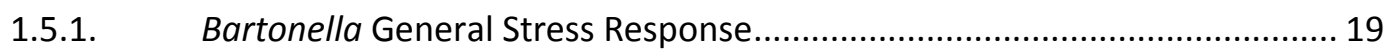

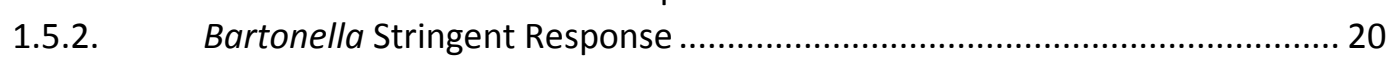

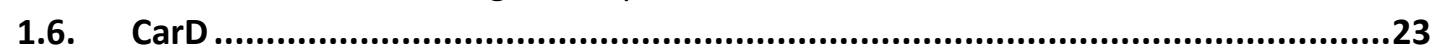

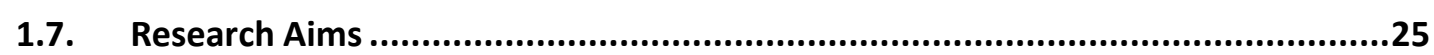

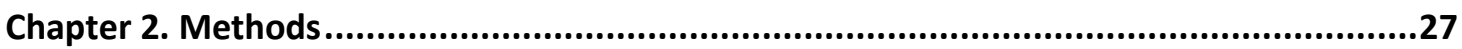

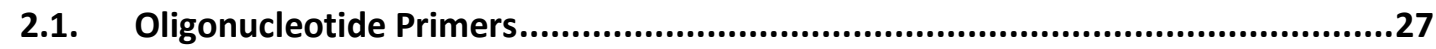

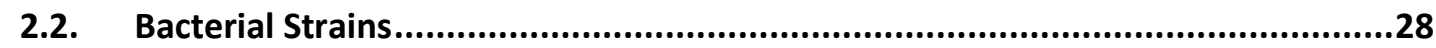

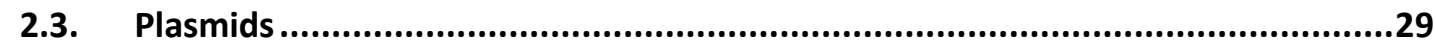

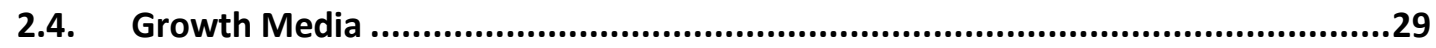

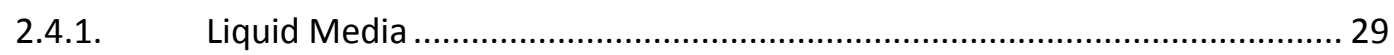

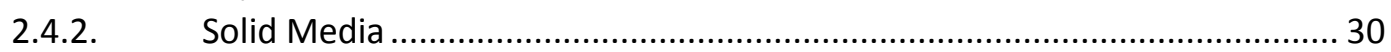

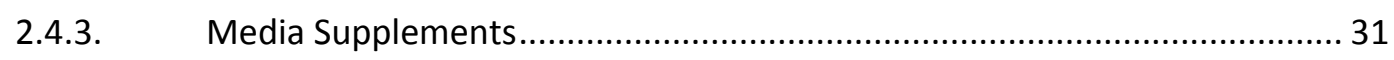

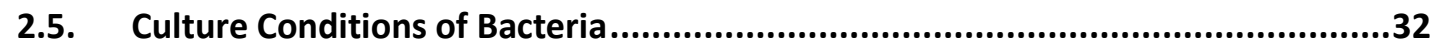

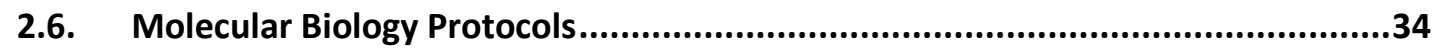

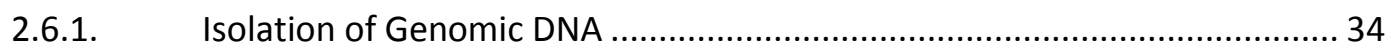

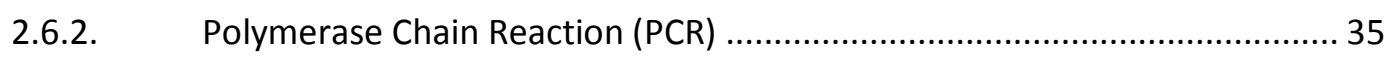

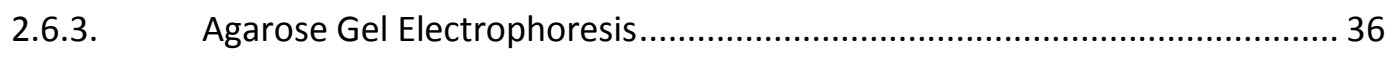

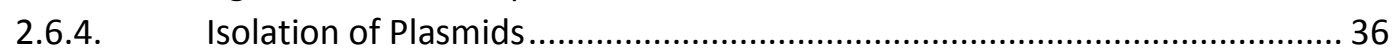

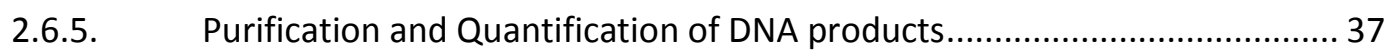

2.6.6. Size-Specific Purification of DNA products by Gel Electrophoresis ............... 37

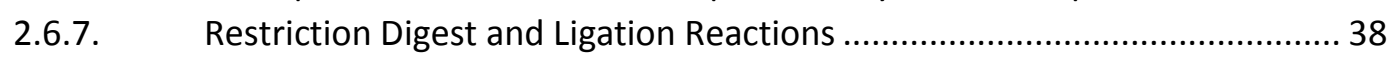

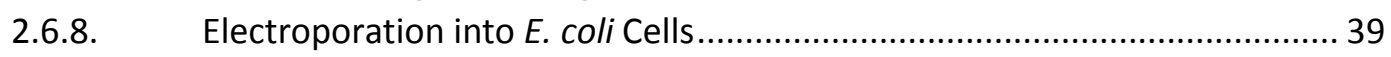

2.6.9. DNA Sequencing and DNA Sequence Analysis ....................................... 40 


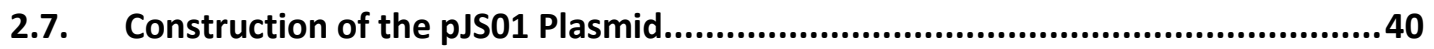

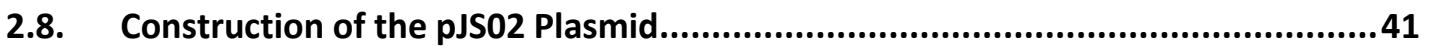

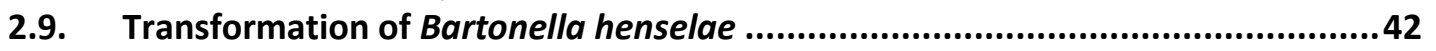

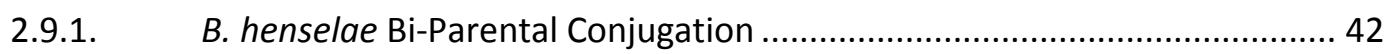

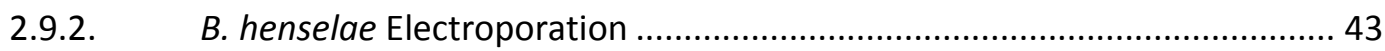

2.10. Growth and Stress Response Assays..........................................................44

2.10.1. Acclimatised B. henselae Bacterial Suspension Preparation....................... 44

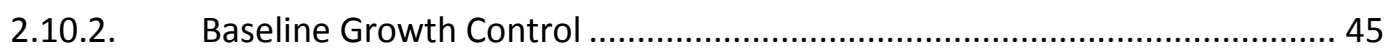

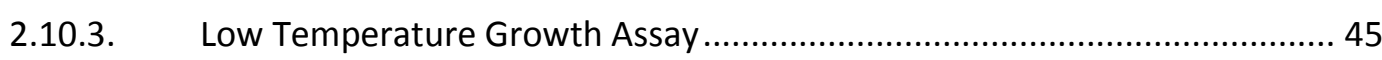

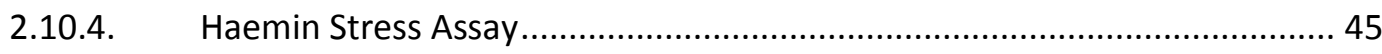

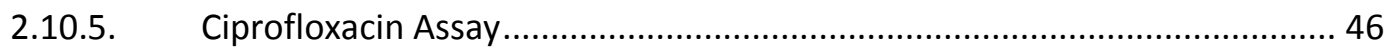

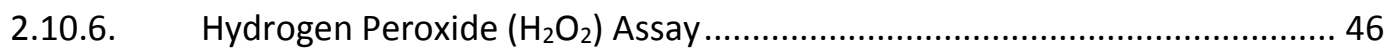

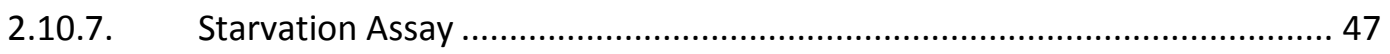

2.11. qPCR Analysis of carD Stress Induced Expression ...........................................47

2.11.1. Selection of $B$. henselae Stress Conditions................................................ 47

2.11.2. Collection of $B$. henselae Cultures under Treatment Conditions .................. 48

2.11.3. B. henselae RNA Extraction using TRIzol and RNeasy ................................. 49

2.11.4. Synthesis of cDNA Using Reverse Transcriptase ........................................ 50

2.11.5. Quality Control of cDNA Products ............................................................ 51

2.11.6. $\quad$ qPCR Quantification of B. henselae carD Expression .................................. 52

Chapter 3. Construction of a Bartonella henselae carD Markerless Deletion Strain............53

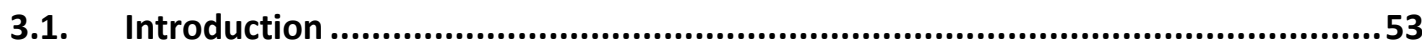

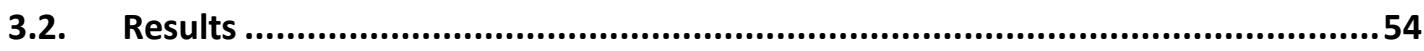

3.2.1. Generation of the Mutagenic Plasmid pJS01 ….......................................... 56

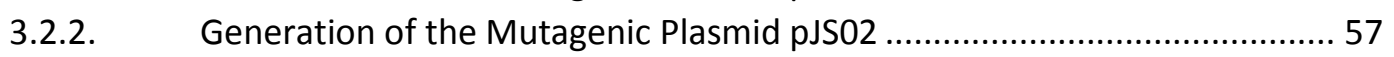

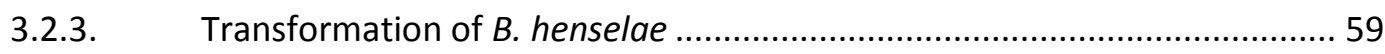

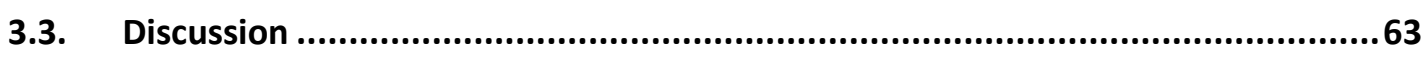

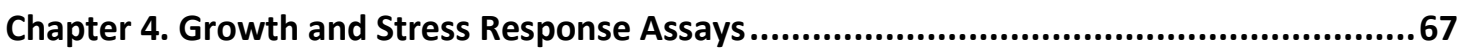

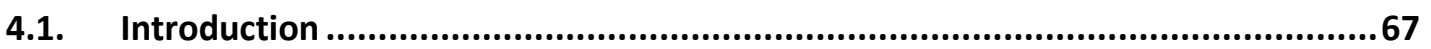

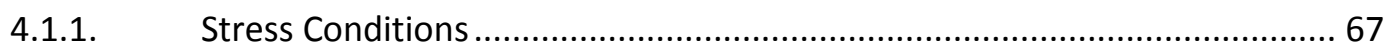

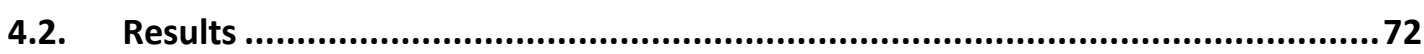

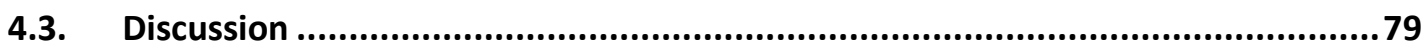

4.3.1. Schneider's Medium Control $\left(37^{\circ} \mathrm{C}\right)$ and Low Temperature $\left(28^{\circ} \mathrm{C}\right)$............. 79

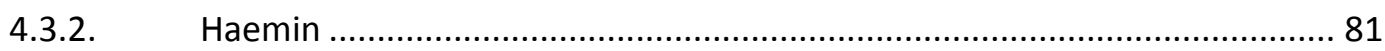

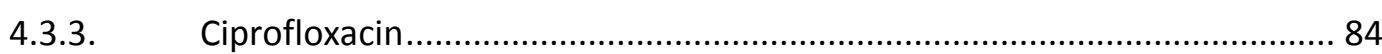

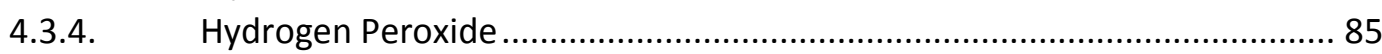

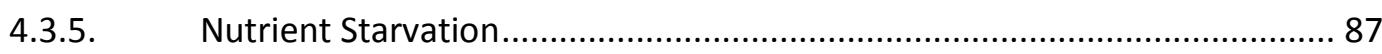

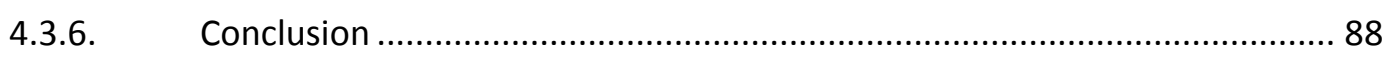

Chapter 5. qPCR Analysis of carD Expression under Stress Conditions .............................89

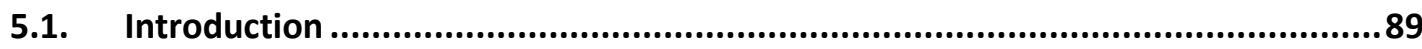

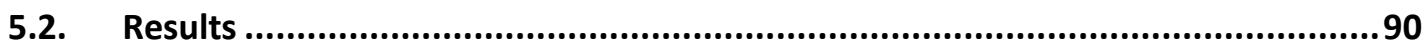

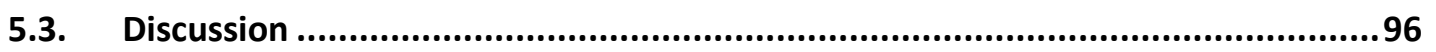

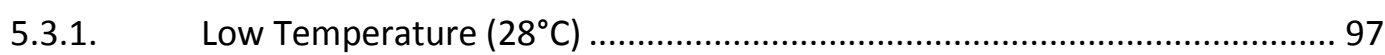

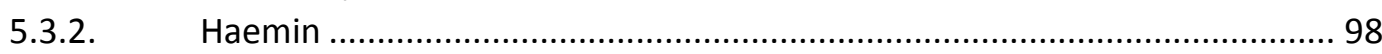

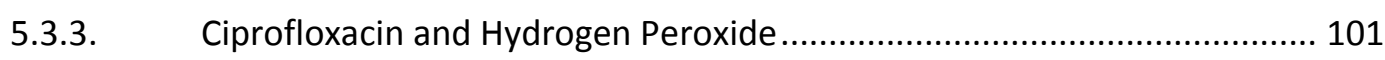

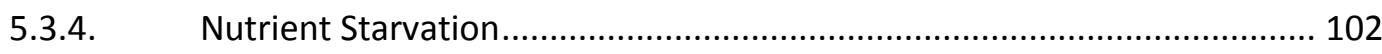

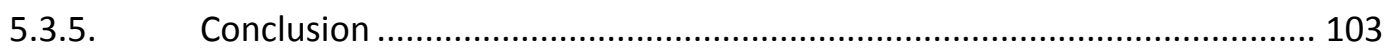


Chapter 6. Research Motivations, Key Findings, and Future Directions ............................105

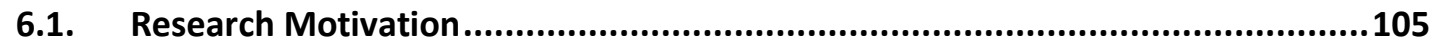

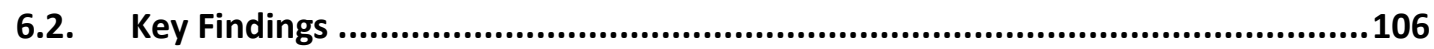

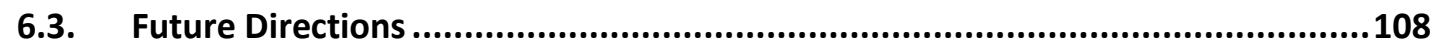

6.3.1. Continuation of our B. henselae $\Delta$ carD Experiments .................................. 108

6.3.1.1. Generation of a B. henselae $\Delta$ carD Mutant Strain .................................... 108

6.3.1.2. Growth Analysis of $B$. henselae Wild-type and $\Delta$ carD Strains during Stress

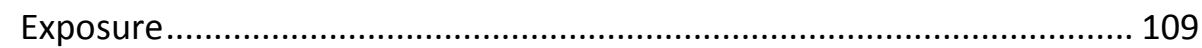

6.3.2. Confirmation of the B. henselae Haemin Response and Further Haemin

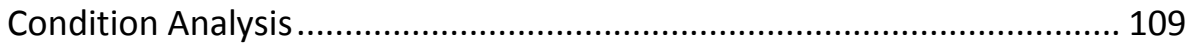

6.3.3. Expansion of the scope of our $B$. henselae carD qPCR assays.................. 110

6.3.3.1. Targeting an Increased Range of $B$. henselae Stress Genes ........................ 110

6.3.3.2. Increase the Scope of our B. henselae carD qPCR Stress Assay .................. 110

6.3.3.3. Testing Stress Conditions that Mimic the B. henselae Environment.......... 111

6.3.4. Studies into the Role of carD during Regular Cellular Function .................. 111

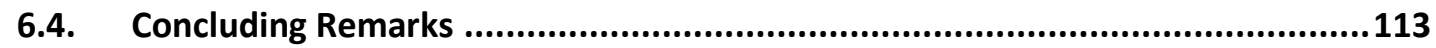

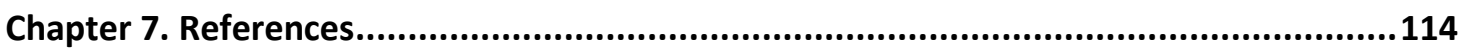

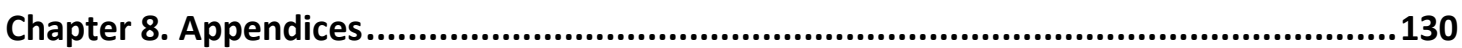

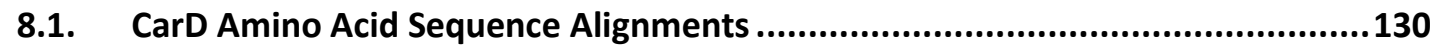

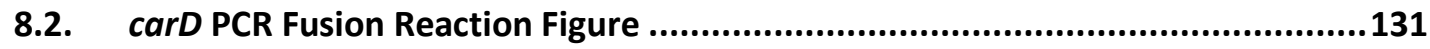

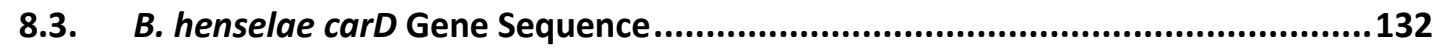

8.4. pJS01 Plasmid Confirmation by Sequencing ..............................................133 


\section{List of Figures}

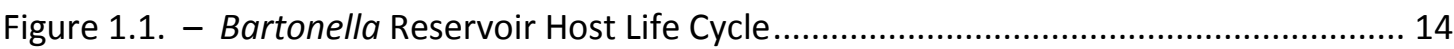

Figure 1.2. - Bartonella general stress response partner switching mechanism................... 20

Figure 1.3. - Hypothetical B. henselae stringent response control over the VirB/D4 and Trw type 4 secretion systems (T4SS) ................................................................. 23

Figure 3.1. - Mutagenesis and selection of the carD mutant ............................................ 55

Figure 3.2. - pEX18gm and pJS01 Agarose Electrophoresis Gels and Bacterial Plasmid Maps

Figure 3.3. - pJM05 and pJS02 Agarose Electrophoresis Gels and Bacterial Plasmid Maps.. 58

Figure 4.1. - Chemical Structure of Porphyrin IX versus Haem B versus Haemin................... 70

Figure 4.2. - Hypothetical Pathway of Haemin/Haem (Heme) Breakdown into Oxidative Products via the Fenton Reaction ................................................................... 70

Figure 4.4. - Growth Curve of $B$. henselae at $37^{\circ} \mathrm{C}$ and $28^{\circ} \mathrm{C}$, Measured in $\mathrm{OD}_{600}$ over 11 Days 74

Figure 4.5. - B. henselae Haemin Stress Exposure Assay, in Percentage Survival, over 24

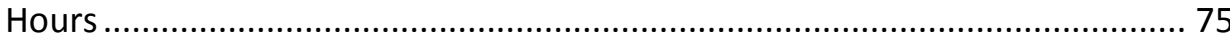

Figure 4.6. - B. henselae Ciprofloxacin Stress Exposure Assay, in Percentage Survival over

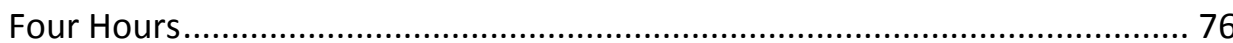

Figure 4.7. - $B$. henselae $\mathrm{H}_{2} \mathrm{O}_{2}$ Stress Exposure Assay, in Percentage Survival over 40 Minutes 77

Figure 4.8. - B. henselae Starvation Stress Exposure Assay, in Percentage Survival over 10 Hours

Figure 5.1. - Agarose Electrophoresis Gels of B. henselae Stress Condition Reverse Transcription Reactions. 92

Figure 5.2. - Agarose Electrophoresis Gel of $B$. henselae $37^{\circ} \mathrm{C}$ qPCR Products 94

Figure 5.3. - Change in Expression ( $\Delta \Delta \mathrm{Ct}$ ) of $c a r D$ Between $37^{\circ} \mathrm{C}$ non-stressed Control and other Stress Conditions using qPCR

Figure 5.4. - Fold Change in Expression of $\operatorname{carD}$ Between $37^{\circ} \mathrm{C}$ Baseline Control and other Stress Conditions using qPCR ......................................................................... 96

Appendix Figure 8.1. - ClustalOmega Amino Acid Sequence Alignments ........................... 130

Appendix Figure 8.2. - Synthesis of the carD PCR fusion products for pJS01 and pJS02 _.... 131

Appendix Figure 8.3. - B. henselae carD sequence +/- 600 basepairs ................................. 132

Appendix Figure 8.4. - Macrogen Sequence Alignments using Geneious ............................ 134 


\section{List of Tables}

Table 1.1. - Human Pathogenic Bartonella Species........................................................ 7

Table 1.2. - Serological B. henselae Prevalence Studies...................................................... 13

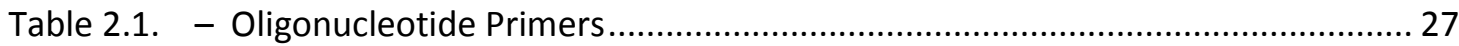

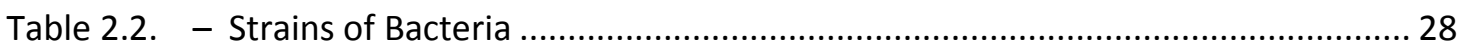

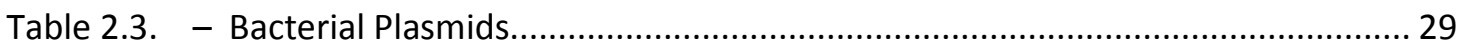

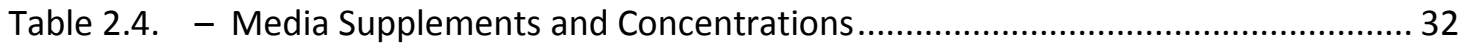

Table 2.5. - Standard PCR Components and Quantities..................................................... 35

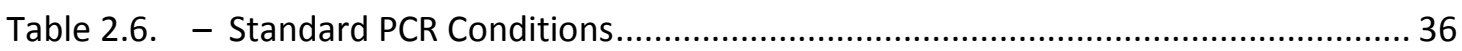

Table 2.7. - Restriction Digest and Ligation Reactions Components and Quantities........... 39

Table 2.8. - B. henselae Stressors and Stressor Conditions............................................... 48

Table 2.9. - Reverse Transcription Reaction Components and Quantities ........................... 51

Table 2.10. - Standard qPCR Components and Quantities ................................................. 52

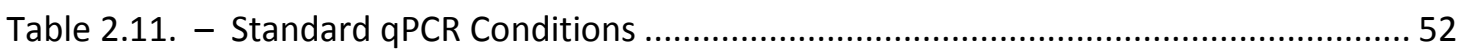

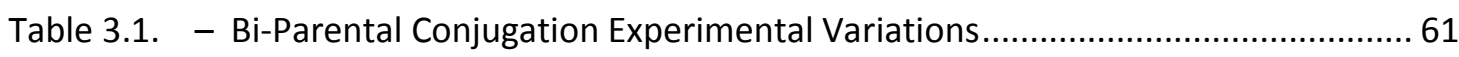

Table 3.2. - B. henselae Electroporation Experimental Variations...................................... 62

\section{Abbreviations used in this thesis}

$\begin{array}{ll}\triangle \text { carD } & \text { carD null mutant strain } \\ \text { cDNA } & \text { Complementary DNA } \\ \text { CSD } & \text { Cat scratch disease } \\ \text { ddH }{ }_{2} \mathrm{O} & \text { Distilled and Deionised } \mathrm{H}_{2} \mathrm{O} \\ \text { EtOH } & \text { Ethanol } \\ \text { gDNA } & \text { Genomic DNA } \\ \text { Gent } & \text { Gentamicin } \\ \text { IFA } & \text { Indirect fluorescent-antibody assay } \\ \text { IL-10 } & \text { Interleukin- } 10 \\ \text { LB } & \text { Luria Broth } \\ \text { M199 } & \text { Media 199 } \\ \text { M199S } & \text { Media } 199 \text { supplemented with fetal bovine calf serum, L-glutamate, and Sodium } \\ & \text { pyruvate } \\ \text { mRNA } & \text { Messenger RNA } \\ \text { OD } D_{x} & \text { Optical density at a wavelength of } x \\ \text { PCR } & \text { Polymerase Chain Reaction } \\ \text { qPCR } & \text { Quantitative Polymerase Chain Reaction } \\ \text { RNA } & \text { Ribonucleic Acid } \\ \text { rRNA } & \text { Ribosomal RNA } \\ \text { SOC } & \text { Super Optimal Broth with added glucose } \\ \text { TRCF } & \text { Transcription repair coupling factor } \\ \text { T4SS } & \text { Type 4 secretion system } \\ \text { WT } & \text { Wild Type }\end{array}$







\section{Chapter 1. Introduction}

In recent years, research has shown that $60.3 \%$ of all emerging infectious diseases are due to zoonotic infections, of which $54.3 \%$ are bacterial in origin ${ }^{[1,2]}$. One group that makes up a large portion of zoonotic bacteria is the genus of Bartonella. Bartonella are fastidious, Gram-negative alphaproteobacteria, capable of intracellular invasion of host tissues. Each Bartonella species has co-evolved to persist in specific host/vector parasitic relationships. Such relationships always involve a warm-blooded mammalian reservoir host and a haematophagous (blood sucking) arthropod vector, responsible for transmission of the pathogen ${ }^{[3]}$. A wide range of mammalian species act as Bartonella reservoir hosts, including canids, felines, ruminants, ungulates, rodents, marsupials, and chiroptera $^{[4,5]}$. Whereas prior to 1993 the Bartonella genus consisted of one species ${ }^{[6]}$, there are now over 40 identified species and likely many more that remain undiscovered.

The Bartonella life cycle begins when the infected haematophagous arthropod vector takes a blood meal from its mammalian host, during which faeces, containing Bartonella, are deposited onto the skin. The bacteria enter the mammalian host body through the skin, infecting several intracellular niches. From these niches, Bartonella is released into the circulatory system, where the bacteria invade red blood cells. Infected host erythrocytes are taken up into the gut of the arthropod vector during a blood meal, restarting the cycle. Though each Bartonella species has evolved to invade only the specific erythrocytes of a mammalian reservoir host, Bartonella species may also cause non-erythrocytic infections in other mammalian species, which are known as incidental hosts. In the life cycle of pathogenic organisms, a reservoir host is a species whose infection will contribute to the life cycle of the pathogen, e.g. B. quintana in humans. In 
contrast, an incidental host is a species whose infection cannot be passed on to another species. Incidental host infection will not contribute to the life cycle of the pathogen, e.g. B. henselae in humans and dogs.

Unlike many other bacterial diseases, Bartonella infections cannot be easily diagnosed using in vitro culturing. The bacteria have a slow growth rate and require specific growth conditions, including haemoglobin enriched media and capnophilic (high carbon dioxide) and microaerophilic (low oxygen) conditions. Bartonella also frequently have a low bacterial load in infected individuals, requiring extra enrichment steps to enable detection. Samples of patient tissue, serum, or whole blood may be incubated in supplemented insect media (such as Schneider's Drosophila Medium supplemented with serum ${ }^{[7]}$, or Bartonella Alpha Proteobacteria Growth Medium, BAPGM ${ }^{[8]}$ ) to enrich bacteria before samples can be analysed to diagnose Bartonella infections ${ }^{[9]}$.

In healthy reservoir hosts, Bartonella infection will usually result in persistent bacteraemia, with bacteria present in the blood and tissues of a host over a period of months or years ${ }^{[10-12]}$. In many cases, a reservoir host infection will remain sub-clinical, although Bartonella infections can predispose otherwise healthy hosts to secondary infections and other health issues ${ }^{[13,14]}$. In young, old and infirm reservoir hosts, and in incidental hosts, Bartonella exposure can lead to long-lasting relapsing infection, known as bartonellosis, resulting in a broad spectrum of medical complications ${ }^{[15]}$. Treatment of Bartonella infection has improved to the point where the correct combination of antibiotics generally resolves the infection - with the right treatment Bartonella infections are rarely fatal. Treatment can vary slightly depending on the species of Bartonella, immune status of the host, whether the infection is chronic or acute, or if 
there are additional complications caused by the infection such as endocarditis or bacillary angiomatosis ${ }^{[16,17]}$. Gentamicin, doxycycline, and rifampin are common choices to treat Bartonella infections, and standard chemotherapy protocol recommendations have been published by clinicians ${ }^{[16]}$.

\subsection{Human-Specific Bartonella Species}

Currently two Bartonella species have been found to use humans as reservoir hosts, B. bacilliformis and B. quintana. Infection with either of these two Bartonella species can result in serious health problems, although risk of infection is low due to the limited prevalence of the relevant arthropod vectors. B. bacilliformis is restricted to the Andes region of South America, the geographic range of its associated sandfly vector, while $B$. quintana is prevalent in lower socioeconomic groups, who suffer elevated exposure to the human body louse vector.

\subsubsection{Bartonella bacilliformis}

B. bacilliformis, described in 1909 , is spread by Lutzomyia sand flies endemic to the Andes region $^{[18]}$. B. bacilliformis was the only member of the Bartonella genus until 1993 when, following the discovery of related species, the Bartonella and Rochalimaea genera were merged ${ }^{[6]}$. B. bacilliformis differs from other species of Bartonella in virulence and lethality, as invasion of host erythrocytes by B. bacilliformis results in the potentially lethal haemolysis of the red blood cells. Untreated infection leads to Verruga peruana (pathological angiogenesis resulting in tumour-like growths on the skin) and Oroya fever (haemolytic anaemia) ${ }^{[16,19]}$. 


\subsubsection{Bartonella quintana}

B. quintana is seen worldwide, and is transmitted by Pediculus humanus, the human body louse. B. quintana is believed to have diverged at some point from B. henselae, although an accurate timescale for such an event is unknown. One hypothesis is that divergence occurred as the human body louse split from the human head louse, $~ 72,000$ years ago ${ }^{[20]}$, around the time when humans began wearing clothing. However, it has been suggested that the level of divergence between $B$. quintana and $B$. henselae indicates an earlier split is more likely ${ }^{[21]}$. The earliest documented evidence of B. quintana infection in humans is found in 4000 year old remains ${ }^{[22]}$. In modern times, the infection rate of $B$. quintana is very low due to the relative rarity of the human body louse ectoparasite; advances in public health, personal hygiene, and more readily available hot water have reduced the prevalence of the parasite, and in turn B. quintana.

B. quintana is responsible for trench fever, a disease characterised by a five day fever cycle, with symptoms that include, but are not limited to, severe headaches, sore muscles, and bone and eye pain ${ }^{[16,19]}$. The largest outbreaks of trench fever were among soldiers serving on the French front in World War $\mathrm{I}^{[23,24]}$, and on the eastern front in World War II ${ }^{[25,26]}$. Trench fever is not usually lethal, but had one of the highest morbidities of any disease over the duration of World War I; of all British troops sent home from the trenches due to sickness in $1917,15.0 \%$ of cases were due to trench fever ${ }^{[26]}$. Both the human body louse and a blood-borne bacterium were proposed as the agents responsible in $1916^{[27,28]}$, and later confirmed in $1918^{[29,30]}$. Since World War II,

prominent $B$. quintana outbreaks have been seen in several African nations ${ }^{[31,32]}$, as well as isolated cases in homeless patients in France, Russia, and the United States ${ }^{[33-36]}$. Urban homelessness, together with alcoholism and intravenous drug addictions, often 
lead to body louse infestations, which enables the transmission of B. quintana $^{[37,38]}$. Coupled with the fact that these populations are also especially vulnerable to contracting HIV/AIDS and related immune deficiency diseases, co-infection of the virus and B. quintana re-emerged in the 1980 s and 1990 s as a new public health problem ${ }^{[12,39]}$. This was characterised by a rise in trench fever, along with bacillary angiomatosis and endocarditis, also due to $B$. quintana ${ }^{[12,40]}$.

\subsection{Zoonotic Bartonella Species}

Zoonotic Bartonella infections in humans are far more prevalent than the reservoir host infections of B. bacilliformis and B. quintana. Currently 16 Bartonella species, along with two candidate species, are known to act as disease causative agents in humans (see Table 1.1), for which a variety of mammals act as hosts, sometimes of multiple species $^{[9,10]}$. Incidental host infections differ from reservoir host infections; Bartonella in incidental hosts colonise similar cellular and tissue sites as in reservoir hosts (excluding erythrocytic cells), but appear to have more evident secondary illnesses associated with infections. The most apparent difference between reservoir and incidental hosts is that in most cases Bartonella in incidental hosts cannot invade erythrocytes, preventing spread through arthropod vectors ${ }^{[41]}$; research has shown that B. henselae invasion of human erythrocytes is possible due to the similarity between B. quintana and B. henselae, but occurs at too low a frequency to enable transmission ${ }^{[42]}$. 


\subsubsection{Groups at Risk of Bartonella Infection}

Exposure to animal-associated Bartonella species generally occurs through contact with animals (e.g., scratches or bites). Exposure to arthropod vectors may play a role, although this remains a heavily debated topic within the field of Bartonella research. Transmission of zoonotic Bartonella is a known occupational hazard for those who interact with animals on a regular basis - veterinarians, animal handlers, farmers, and pet owners are at heightened risk.

Also at a possible heightened risk are those who receive blood transfusions, in the United States in 2011 around 40,000 units of whole blood or red blood cells were collected, with 38,000 units transfused ${ }^{[43]}$. The American Food and Drug Administration mandates that red blood cells can be refrigerated for a maximum of 42 days, yet B. henselae remains viable in stored red blood cell units for 35 days ${ }^{[44,45]}$. B. henselae is transmissible through needle pricks in humans ${ }^{[46,47]}$, and is transmissible through intravenous blood transfusions in both mice and cat animal models ${ }^{[48-50]}$. This raises the question as to whether $B$. henselae, or other Bartonella species, may be transmitted between human hosts through blood transfusions. Prevalence studies of Bartonella in blood donors are limited, with the majority focused on $B$. henselae (see Table 1.2) the seroprevalence (number of patients whose serum tested positive for Bartonella antibodies) of $B$. henselae in blood donors varies between countries, ranging from $0.6 \%$ to $34.4 \%$, but the average worldwide is about $10.0 \%$, making Bartonella transmission via blood transfusions a notable risk for recipients. 
Table 1.1. - Human Pathogenic Bartonella Species All Bartonella species listed here (with the exception of those in bold) are zoonotic human pathogens. An example of reservoir and vector host species is noted for each Bartonella species.

\begin{tabular}{|c|c|c|c|c|}
\hline Bartonella spp. & Reservoir spp. & Vector spp. & Incidental spp. & \\
\hline B. alsatica ${ }^{[51]}$ & $\begin{array}{l}\text { Rabbits- } \\
\text { O. cuniculus }\end{array}$ & $\begin{array}{l}\text { Rabbit Flea- } \\
\text { S. cuniculi? }\end{array}$ & Human & \\
\hline B. bacilliformis ${ }^{[52]}$ & $\begin{array}{l}\text { Humans- } \\
\text { H.sapiens }\end{array}$ & $\begin{array}{l}\text { Sandfly- } \\
\text { L. verrucarum }\end{array}$ & None & \\
\hline B. clarridgeiae ${ }^{[53]}$ & $\begin{array}{l}\text { Cats- } \\
\text { F. catus }\end{array}$ & $\begin{array}{l}\text { Cat Flea- } \\
\text { C. felis }\end{array}$ & Human, Dog & \\
\hline B. doshiae $e^{[54]}$ & $\begin{array}{l}\text { Voles and Rats- } \\
\text { M. agentiis }\end{array}$ & $\begin{array}{l}\text { Rodent Flea- } \\
X . \text { cheopis? }\end{array}$ & Human & \\
\hline B. elizabethae $e^{[55]}$ & $\begin{array}{l}\text { Rats- } \\
\text { R. norvegicus }\end{array}$ & $\begin{array}{l}\text { Oriental Rat Flea- } \\
X \text { cheopis }\end{array}$ & Human, Dog & \\
\hline B. grahamii ${ }^{[56]}$ & $\begin{array}{l}\text { Mice- } \\
\text { C. glareolus }\end{array}$ & $\begin{array}{l}\text { Rodent Flea- } \\
X . \text { cheopis? }\end{array}$ & Human & \\
\hline B. hensela $e^{[56]}$ & $\begin{array}{l}\text { Cats- } \\
\text { F. catus }\end{array}$ & $\begin{array}{l}\text { Cat Flea- } \\
\text { C. felis }\end{array}$ & $\begin{array}{l}\text { Human, Dog, } \\
\text { Porpoise }\end{array}$ & Horse, \\
\hline B. koehlerae $e^{[56]}$ & $\begin{array}{l}\text { Cats- } \\
\text { F. catus }\end{array}$ & $\begin{array}{l}\text { Cat Flea- } \\
\text { C. felis }\end{array}$ & Human, Dog & \\
\hline B. melophagi[57] & $\begin{array}{l}\text { Sheep- } \\
\text { O. aries }\end{array}$ & $\begin{array}{l}\text { Sheep Ked- } \\
\text { M. ovinus }\end{array}$ & Human & \\
\hline B. quintana ${ }^{[58]}$ & $\begin{array}{l}\text { Humans- } \\
\text { H. sapiens }\end{array}$ & $\begin{array}{l}\text { Human Body Louse- } \\
\text { P. humanis }\end{array}$ & Cat, Dog & \\
\hline B. rattimassiliensis ${ }^{[59]}$ & $\begin{array}{l}\text { Rats- } \\
\text { R. rattus? }\end{array}$ & $\begin{array}{l}\text { Rat Flea- } \\
X . \text { cheopis? }\end{array}$ & Human & \\
\hline B. rochalimae $e^{[55]}$ & $\begin{array}{l}\text { Canids- } \\
\text { C. familiaris }\end{array}$ & $\begin{array}{l}\text { Dog Fleas- } \\
\text { P. irritans }\end{array}$ & Human, Dog, Cat & \\
\hline B. schoenbuchensis ${ }^{[54]}$ & $\begin{array}{l}\text { Deer- } \\
\text { C. capreolus }\end{array}$ & $\begin{array}{l}\text { Deer Ked- } \\
\text { L. cervi }\end{array}$ & Human & \\
\hline B. tamiae ${ }^{[60]}$ & $\begin{array}{l}\text { Rats- } \\
\text { R. rattus? }\end{array}$ & $\begin{array}{l}\text { Chigger Mites and Ticks- } \\
\text { Trombiculidae }\end{array}$ & Humans & \\
\hline B. tribocorum ${ }^{[54]}$ & $\begin{array}{l}\text { Rats- } \\
\text { R. rattus }\end{array}$ & $\begin{array}{l}\text { Rat Flea- } \\
X . \text { cheopis? }\end{array}$ & Human & \\
\hline B. vinsonii arupensis ${ }^{[56]}$ & $\begin{array}{l}\text { Mice- } \\
\text { Pleucopu }\end{array}$ & $\begin{array}{l}\text { Unknown- } \\
?\end{array}$ & Human & \\
\hline B. vinsonii berkhoffii ${ }^{[56]}$ & $\begin{array}{l}\text { Canids- } \\
\text { C. latrans }\end{array}$ & $\begin{array}{l}\text { Unknown- } \\
?\end{array}$ & Human, Cat & \\
\hline B. washoensis ${ }^{[61]}$ & $\begin{array}{l}\text { Squirrel- } \\
\text { S. beecheyii }\end{array}$ & $\begin{array}{l}\text { Rodent Flea- } \\
\text { O. montana }\end{array}$ & Human, Dog & \\
\hline $\begin{array}{l}\text { B. ancashi[62] } \\
\text { (Canditatus) }\end{array}$ & $\begin{array}{l}\text { Unknown- } \\
?\end{array}$ & $\begin{array}{l}\text { Sandfly- } \\
\text { L. verrucarum? }\end{array}$ & Human & \\
\hline $\begin{array}{l}\text { B. mayotimonensis } \\
\text { (Canditatus) }\end{array}$ & $\begin{array}{l}\text { Bats?- } \\
?\end{array}$ & $\begin{array}{l}\text { Bat Flies and Fleas- } \\
?\end{array}$ & Human & \\
\hline
\end{tabular}




\subsection{Bartonella henselae}

The most prevalent zoonotic Bartonella species is B. henselae, the causative agent of cat scratch disease (CSD), as well as several other illnesses. Due to genomic similarities, it has been suggested to be the progenitor species of $B$. quintana ${ }^{[21]}$. The reservoir host of $B$. henselae is the house cat, Felis catus, and B. henselae is transmitted between cats by the faeces or flea 'dirt' of the cat flea, Ctenocephalides felis. The majority of human B. henselae infections are due to cat bites and scratches ${ }^{[64-66]}$, and is rarely transmitted to humans through flea faeces ${ }^{[67,68]}$. Though most cases of zoonotic $B$. henselae infection are in humans, $B$. henselae has also been known to infect dogs, horses, and porpoises as secondary hosts ${ }^{[69,70]}$.

\subsubsection{History of $B$. henselae and Cat Scratch Disease}

Previously undocumented in modern medicine, CSD was identified independently in patients in 1931 by Robert Debré in France, and in 1932 by Lee Foshay in the United States; the pair met in $1947^{[71]}$, leading to the first published clinical description of CSD in French by Debré in $1950^{[72]}$. The first report of CSD written in English was by Greer et al, who presented findings to medical professionals at the American Clinical and Climatological Association in late 1950 and published in $1951^{[71,73]}$. Numerous studies have been published since 1950 , describing case studies, epidemiology ${ }^{[74-76]}$, related diseases $^{[77]}$, and transmission to animals ${ }^{[71]}$. However, CSD aetiology remained unknown until a bacterial presence was noted in patients whose immune systems were suppressed by HIV/AIDS in the $1980 \mathrm{~s}^{[78]}$. A Gram negative, rod-shaped bacterium, later identified as $B$. henselae, was identified in histological sections of a Warthin-Starry stained lymph node from a CSD patient in $1983^{[79]}$. The bacterium was first linked to the aetiology of bacillary angiomatosis ${ }^{[80]}$, bacillary peliosis hepatis ${ }^{[81]}$, and fever and 
bacteraemia in $1990^{[82]}$, before being shown as the causative agent of CSD in $1992^{[83,84]}$. The bacterium was isolated and characterised as Rochalimaea henselae in $1992^{[85,86]}$, then renamed Bartonella henselae and merged into the Bartonella genus alongside B. quintana and the related zoonotic pathogens, B. elizabethae and B. vinsonii, in $1993^{[87]}$.

\subsubsection{Bartonella henselae, Felis catus, and Ctenocephalides felis}

The housecat is a common pet, with an ownership average (defined as the percentage of households with at least one cat) of $25.0 \%$ for Europe ${ }^{[88]}, 35.0 \%$ for North America ${ }^{[89,90]}$, and $49.0 \%$ in New Zealand ${ }^{[91]}$. B. henselae prevalence is highest in cat populations that have a high rate of flea infestations; seroprevalence is greatest in young and stray cats $^{[92,93]}$, and in regions with a warm climate and moderate precipitation ${ }^{[94]}$. Cats infected with $B$. henselae often suffer subclinical, persistent bartonellosis ${ }^{[10]}$, but infection can alternatively result in clinical manifestations or predispose the affected cat to a number of ailments ${ }^{[13,14]}$.

Ctenocephalides felis (C. felis) is the primary ectoparasite of both cats and dogs worldwide ${ }^{[95-97]}$; under the right conditions, over $90.0 \%$ of cats can be infected by C. felis in a population of feral cats ${ }^{[98,99]}$. Areas with a warm climate and moderate precipitation favour C. felis, with fleas growing faster and larger ${ }^{[100]}$. Adult $C$. felis act as the haematophagous vector of $B$. henselae infection in cat-to-cat transmissions. Fleas living on bacteremic cats were shown to harbour bacteria in $1994^{[101]}$, and to transmit B. henselae in $1996^{[102,103]}$. As in the majority of arthropod vectors, fleas take up B. henselae during a blood meal from an infected cat. Erythrocytes are lysed in the arthropod midgut, where Bartonella survives exposure to high levels of haemin, persists and replicates. Voided gut contents and dirt may be culture positive for $B$. henselae, 
with bacteria harvested from flea faeces remaining viable for three days ${ }^{[102,104,105]}$. Both voided gut contents and dirt are crucial for the flea life cycle, acting as nutrients for larvae ${ }^{[106]}$, and providing a possible method of flea-to-flea transmission of Bartonella ${ }^{[107]}$. Currently, B. henselae are known to maintain themselves for 12 days in the $C$. felis midgut, the entire lifespan of fleas fed from artificial blood-membrane system $^{[108]}$. No studies investigating B. henselae persistence in fleas fed on animals have been performed, where $C$. felis can live and reproduce up to a hundred days ${ }^{[109]}$.

\subsubsection{Cat Scratch Disease}

In 1997, 24,000 people were diagnosed with CSD in the United States; $80.0 \%$ of these were children between two and 14 years old ${ }^{[76,110,111]}$. Cases of CSD increase in autumn and winter, potentially due to the increased time cats spend indoors and in contact with humans $^{[112]}$. Previously, CSD in humans was believed to be a self-limiting disease, although recent studies show that this may not always be the case ${ }^{[11,113-115]}$. In a study of 24 patients with severe CSD related arthropathy (joint pain), $80.0 \%$ of patients fully recovered within six weeks, while the remainder had chronic disease, lasting between 16 and 53 months ${ }^{[116]}$.

Around $85.0 \%$ of CSD patients suffer from 'typical' CSD symptoms ${ }^{[117]}$, which involve a raised bump or lesion on the site of infection, followed by lymphadenopathy (swelling of the lymph node nearest to the site) and minor symptoms also typical of other Bartonella infections ${ }^{[118]}$. Bartonellosis symptoms vary between patients depending on health and age. Common symptoms are often non-specific, including chronic fatigue, malaise, headaches, acute fever, mygalia (muscle pain), arthralgia (joint pain), paresthesia (loss of sensation), photophobia (sensitivity to light), and memory 
loss. In the remaining $15.0 \%$ of 'atypical' CSD infections, and especially in immunocompromised patients, a range of neurological and major organ symptoms may appear, including bacillary angiomatosis (formation of tumour-like lesions), pericarditis and endocarditis (inflammation of the pericardium and endocardium heart tissue), pleural and pericardial effusion (build-up of fluid around the lungs and heart), bacillary splenitis (lesion of the spleen), peliosis hepatis (blood filled spaces in the liver), meningitis (inflammation of membranes surrounding the brain and spinal cord), myelitis (inflammation of the spinal cord), neuroretinitis (inflammation of the retina or optic nerve), cerebral arteritis and artery occlusion (inflammation of arteries in the brain and blockage of arteries), seizures and convulsions, and hemiplegia (paralysis) ${ }^{[119-131]}$. Bartonella species have also been named as a probable causative agent of cerebral bacillary angiomatosis ${ }^{[132]}$, and of dementia in HIV-positive patients ${ }^{[133,134]}$.

\subsubsection{Prevalence of Bartonella henselae}

Studies have been undertaken in the last 25 years to determine the prevalence of Bartonella species in humans and animals worldwide. In particular, the prevalence of B. henselae has been assessed using indirect fluorescent-antibody (IFA) assays. Other techniques, such as PCR, have become more common as bacterial enrichment techniques have improved. IFA assays detect Bartonella genus- or species-specific $\operatorname{IgG}$ or IgM antibodies in the patient's bloodstream, to determine if a patient has been exposed to the bacteria, giving a snapshot of the prevalence of Bartonella in a sample population. IFA results will only be positive if the correct antibodies are present, although the level of each antibody appears to fluctuate over the course of the disease; levels of $\operatorname{IgM}$ decrease within three months after initial infection, while $\operatorname{IgG}$ levels remain detectable for several years following infection ${ }^{[135,136]}$. Combined with PCR and 
culturing techniques, IFA serology is accurate at diagnosing Bartonella infections; however, previous patient exposure to Bartonella can give false positives if serology is the sole diagnosis technique used.

Seroprevalence varies considerably within country (depending on climate, urban versus rural location, population of humans and cats etc). Serological studies are a good starting point for determining the prevalence of Bartonella. Interestingly, the majority of B. henselae seroprevalence studies currently are performed in healthy adults and blood donors, rather than in children, who are the main sufferers of CSD. Table 1.2 demonstrates that seroprevalence of $B$. henselae follows several trends, with some exceptions. High seroprevalence is seen in countries with warm, wet climates and in those that have a high cat population (domesticated or stray). Climate appears to be a large factor in seroprevalence, most likely due to favourable conditions for $C$. felis. The exceptions to these trends are in Germany and Canada, where despite lower temperatures (and Germany's low cat ownership) both nations have higher seroprevalence than similar nations. Unexpectedly high seroprevalence levels can likely be explained by how animals are regarded in a community, and the laws and regulations of a nation - a society that has a positive attitude towards stray cats will have higher interactions with the animals (e.g. feeding and socialisation), which can result in increased rate of infection. Conversely, negligence in caring for pet cats, in controlling the cat population, or in dealing with abandoned pets may all increase the rate of infection $^{[89,137,138]}$. New Zealand has a reported seroprevalence of 5.0\% and a household cat ownership of $49.0 \%^{[89]}$; of cats tested in Auckland, $17.0 \%$ were culture positive for B. henselae, and of C. felis from three North Island Veterinaries, $11.0 \%$ were PCR positive for B. henselae $e^{[139,140]}$. 
Table 1.2. - Serological B. henselae Prevalence Studies All serology studies were performed using IFA serology. $\mathrm{Bd}=$ Blood donors, $\mathrm{Vs}=$ Veterinarian students, $\mathrm{Ha}=$ Healthy adults, $\mathrm{Hc}=$ Healthy children. Cat ownership per country is given as an estimate of the percentage of households that own at least one cat. N/A signifies the country currently has no reliable cat population statistics available.

\begin{tabular}{|c|c|c|c|c|c|c|}
\hline \multicolumn{2}{|l|}{ Prevalence } & \multirow{2}{*}{$\begin{array}{r}\text { Country } \\
\text { Denmark }\end{array}$} & \multirow{2}{*}{$\begin{array}{c}\text { Sample } \\
\text { Size }\end{array}$} & \multirow{2}{*}{$\begin{array}{l}\begin{array}{l}\text { Group } \\
\text { Studied }\end{array} \\
\text { Bd }\end{array}$} & \multirow{2}{*}{$\begin{array}{l}\text { Year } \\
2004^{[141]}\end{array}$} & \multirow{2}{*}{$\begin{array}{c}\text { Cat Ownership } \\
\text { (Household) }\end{array}$} \\
\hline \multirow{6}{*}{$\leq 5.0 \%$} & $0.6 \%$ & & & & & \\
\hline & $1.2 \%$ & Sweden & 498 & $\mathrm{Bd}$ & $2005^{[142]}$ & $17.0 \%^{[88]}$ \\
\hline & $1.5 \%$ & UK & 200 & $\mathrm{Bd}$ & $1999^{[143]}$ & $19.0 \%^{[88]}$ \\
\hline & $4.5 \%$ & Japan & 155 & Vs & $2001^{[144]}$ & $\mathrm{N} / \mathrm{A}$ \\
\hline & $5.0 \%$ & New Zealand & 140 & $\mathrm{Bd}$ & $2007^{[145]}$ & $49.0 \%^{[91]}$ \\
\hline & $5.0 \%$ & USA & 199 & $\mathrm{Bd}$ & $1996^{[36]}$ & $35.0 \%^{[90]}$ \\
\hline \multirow{3}{*}{$\leq 10.0 \%$} & $5.5 \%$ & Thailand & 163 & $\mathrm{Bd}$ & $2000^{[146]}$ & N/A \\
\hline & $6.0 \%$ & Turkey & 800 & $\mathrm{Bd}$ & $2009^{[147]}$ & $9.0 \%{ }^{[88]}$ \\
\hline & $8.7 \%$ & Spain & 218 & $\mathrm{Ha}$ & $2008^{[148]}$ & $19.0 \%^{[88]}$ \\
\hline \multirow{4}{*}{$\leq 15.0 \%$} & $11.0 \%$ & Jordan & 482 & $\mathrm{Hc}$ & $2004^{[149]}$ & N/A \\
\hline & $13.0 \%$ & Germany & 116 & $\mathrm{Hc}$ & $2001^{[150]}$ & $13.0 \%{ }^{[88]}$ \\
\hline & $13.7 \%$ & Brazil & 437 & $\mathrm{Bd}$ & $2005^{[151]}$ & N/A \\
\hline & $14.8 \%$ & China & 351 & $\mathrm{Ha}$ & $2010^{[152]}$ & $4.1 \%^{[153]}$ \\
\hline \multirow{3}{*}{$\leq 30.0 \%$} & $21.6 \%$ & Greece & 491 & $\mathrm{Bd}$ & $2008^{155]}$ & $13.0 \%{ }^{[88]}$ \\
\hline & $24.7 \%$ & Spain & 146 & $\mathrm{Bd}$ & $2005^{[154]}$ & $19.9 \%^{[88]}$ \\
\hline & $30.0 \%$ & Germany & 270 & $\mathrm{Ha}$ & $1998^{[155]}$ & $13.0 \%^{[88]}$ \\
\hline \multirow{2}{*}{$\leq 40.0 \%$} & $34.4 \%$ & Brazil & 125 & $\mathrm{Bd}$ & $2010^{[156]}$ & N/A \\
\hline & $36.8 \%$ & Canada & 142 & $\mathrm{Ha}$ & $2000^{[157]}$ & $37.0 \%{ }^{[89]}$ \\
\hline \multirow{2}{*}{$\geq 50.0 \%$} & $50.0 \%$ & Croatia & 100 & $\mathrm{Bd} / \mathrm{Hc}$ & $2009^{[158]}$ & N/A \\
\hline & $61.6 \%$ & Italy & 508 & $\mathrm{Hc}$ & $2004^{[159]}$ & $19.0 \%^{[88]}$ \\
\hline
\end{tabular}




\subsection{Pathology and Host Cell Interactions of Bartonella}

\subsubsection{Bartonella Life Cycle}

The life cycle of Bartonella in reservoir hosts can be characterised by two major phases, the vector phase and the host phase. Though the vector and host vary for each Bartonella species, the life cycle, as shown below in Figure 1.1 is largely similar for all members of the genus, with the exception of $B$. bacilliformis.

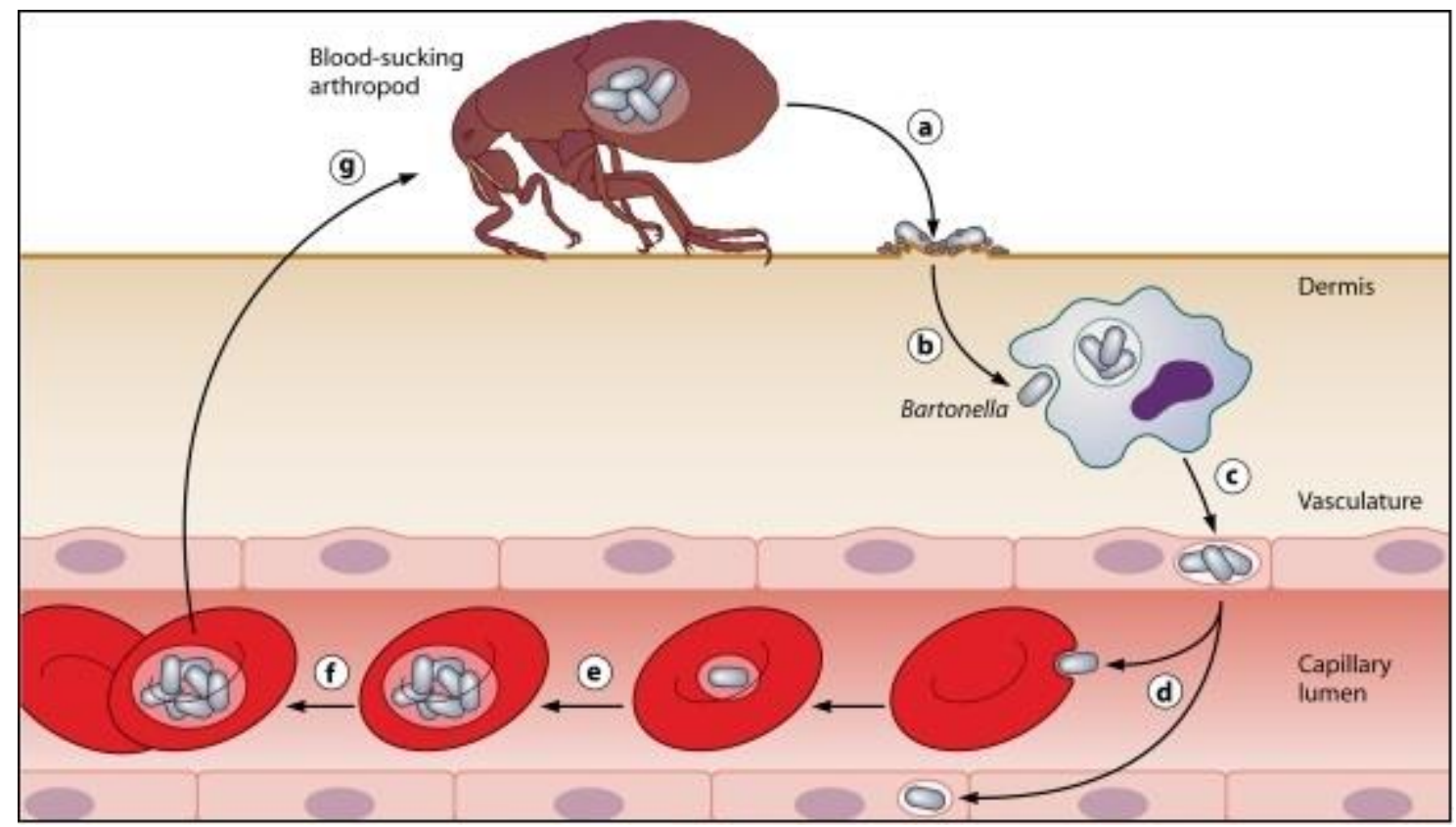

Figure 1.1. - Bartonella Reservoir Host Life Cycle a) Bacteria are deposited on the skin of the mammalian host. b) Bartonella enter the host and are likely taken up by migratory cells. c) Transport to the vascular endothelium occurs, where the bacteria are thought to persist intracellularly. d) From endothelial and other infected cells, the bacteria are seeded into the bloodstream, where they invade erythrocytes and possibly other cell types. e and f) After limited replication inside the red blood cell, Bartonella persist in the intra-erythrocytic niche. g) Red blood cells are taken up into the haematophagous arthropod vector to restart the cycle. Figure has been reproduced from: Clinical Microbiology Review, 25(1), Harms and Dehio, Intruders below the radar: molecular pathogenesis of Bartonella spp, pages 42-78, 2012, with permission from the American Society for Microbiology ${ }^{[19]}$.

In the first phase, erythrocytes containing Bartonella are taken up by a haematophagous arthropod vector during a blood meal from an infected mammalian host. Lysis of erythrocytes releases bacteria into the arthropod gut, where Bartonella replicates for a 
sustained period of time. The bacteria remain viable in both regurgitated gut contents and faeces of the vector. Transmission to a new mammalian host occurs when the infected arthropod takes a blood meal; the arthropod gut expands during feeding, expelling bacteria-laden faeces onto the skin of the new host. Bartonella enters the new host via abrasions in the skin, often assisted by the host scratching the affected area.

After infiltrating the host, Bartonella enters the second phase, moving through the body, using the blood and lymph systems as vehicles to reach deeper host tissues, possibly via dendritic cells ${ }^{[160,161]}$. As the bacteria move through the circulatory system, Bartonella spp. bind and invade immunologically privileged sites, known as the primary or replicative niches ${ }^{[41]}$. A hotly debated topic, many cellular sites have been proposed to be the primary niche, with vascular, endothelial, microglia, and hematopoietic progenitor cells all suggested as candidates ${ }^{[19,162,163]}$. Within these cellular niches, Bartonella replicate and influence cellular function and expression. Bartonella can invade multiple cell types in vitro, including endothelial, epithelial, hematopoietic progenitor, dendritic, microglial, and monocytic cells ${ }^{[163-166]}$. As of yet, the in vivo relevance has not been fully elucidated for all these cell types.

From the primary niche, Bartonella are proposed to be periodically reseeded into the circulatory system in recurring synchronous waves of infection ${ }^{[41,167]}$, yet this release has not been noted in all species. In the circulatory system, Bartonella bind to and invade erythrocytes; Bartonella has been shown to replicate within the erythrocytes of reservoir hosts, although the number of bacteria per erythrocyte is species specific and limited - an average of eight $B$. tribocorum bacteria per erythrocyte in rats, five $B$. quintana per erythrocyte in humans, and just one $B$. henselae bacterium per erythrocyte in cats ${ }^{[19,41]}$. The proportion of erythrocytes infected in the blood of the reservoir host is also limited, 
with around $1.0 \%$ of all erythrocytes infected in B. quintana and B. tribocorum, and up to $5.0 \%$ in $B$. henselae. These figures differ greatly in B. bacilliformis, which exhibits traits similar to malaria, in that the percentage of infected erythrocytes can reach up to $100.0 \%$, and replication within the erythrocyte will continue until haemolysis ${ }^{[19]}$. Though haemolysis in common in B. bacilliformis, no other Bartonella species has been shown to severely disrupt reservoir host erythrocyte function; erythrocytes remain infected and function for their lifespan or until taken up by a haematophagous vector during a blood meal, restarting the cycle ${ }^{[41]}$.

\subsubsection{Pathology and Mechanism of Infection of B. henselae}

Although infection with $B$. henselae and other Bartonella species leads to clinical symptoms in some reservoir and in most incidental hosts, infection in a healthy reservoir host will often be at sub-clinical levels. During infection of a reservoir host, Bartonella can remain undetected for prolonged periods of time, this is due to both its ability to evade and subvert the immune system of a host, and its ability to hide in immunologically protected sites.

One means of immune evasion is through the unique characteristics of Bartonella lipopolysaccharide. Normally bacterial lipopolysaccharide interacts with host pattern recognition receptors to elicit a large immune response, but Bartonella lipopolysaccharide differs in structure, leading to a much reduced binding rate to host Toll-like receptor 4, relative to other Gram negative bacterial pathogens ${ }^{[168,169]}$. There is evidence that Bartonella also undergoes phase and antigenic variation as a means of avoiding the adaptive immune response ${ }^{[170]}$. Bartonella also actively modulates the immune system, through mechanisms not fully understood, stimulating secretion of high levels of the cytokine IL-10, a key regulator of immunity. IL-10 suppresses the functions 
of various immune cells, interfering with innate immunity and the establishment of an adaptive immune response. IL-10 $10^{-/-}$knockout mice infected with $B$. birtlesii did not become bacteraemic ${ }^{[171]}$, demonstrating the crucial role of IL-10 expression in Bartonella infection. Bartonella trimeric auto-transporter adhesins and the VirB/D4 type 4 secretion system (T4SS) may also affect the immune system via host cell interactions ${ }^{[19,168]}$. The most widely studied trimeric auto-transporter adhesin, the B. henselae Bartonella Adhesin A, BadA, governs cell binding and also promotes angiogenesis through up-regulation of hypoxia inducible factor $1^{[18,170,172]}$, and the immune system through $\mathrm{NF}-\mathrm{KB}^{[173]}$, suggesting that other trimeric auto-transporter adhesins could fulfil a similar function.

In the circulatory system, interactions between $B$. henselae and endothelial cells are mediated by BadA. BadA facilitates cellular invasion by first promoting autoaggregation of $B$. henselae, and by adhering to endothelial cells and the extracellular $\operatorname{matrix}^{[19,170,174]}$. Once $B$. henselae adheres to a host endothelial cell, the VirB/D4 T4SS is used to 'inject' Bartonella effector proteins (Beps) into the target cell, bypassing the bacterial inner and outer membranes and the host cell membrane, and additionally preventing endothelial cell apoptosis ${ }^{[175]}$. Though not all of their functions have been discovered, the Beps generally target and modify host cell signalling; some Beps have anti-apoptotic and pro-angiogenic functions ${ }^{[176]}$, while others mediate and assist in the uptake of Bartonella into the cell ${ }^{[177]}$. There are two ways for an endothelial cell to take up B. henselae, either by endocytosis of a single bacterium into a Bartonella-containing vacuole or by uptake of an bacterial aggregate mass into an invasome, via the Beps (e.g. BepG prevents endocytosis, resulting in accumulation of the aggregate mass) ${ }^{[177]}$. Bartonella-containing vacuoles have been found to lack typical endocytic markers such 
as Lamp1, preventing fusion with lysosomes ${ }^{[178]}$. B. henselae has been proposed to replicate within the primary niche and be released back into the circulatory system.

Within the circulatory system, Bartonella species-specific binding to reservoir host erythrocytes is mediated by the Trw T4SS ${ }^{[17,41,175,176]}$; when deleted in $B$. henselae, bacteria are found to be present in the bloodstream, but cannot invade reservoir host erythrocytes $^{[175]}$.

\subsection{Bartonella Stress Response}

To survive and thrive in a given environment, bacteria must overcome stresses; a 'stress' is a broad label for anything that puts pressure on the bacteria - high or low temperatures, $\mathrm{pH}$, nutrient deprivation, competition with other organisms, and antibiotic assault are common stresses. In pathogenic bacteria, the ability to adapt to diverse environments and their respective stresses is paramount to survival; in the Bartonella life cycle these environments occur in the arthropod midgut and the mammalian host circulatory system or intracellular niches. Stresses in the arthropod midgut include a high concentration of haemin (an iron chelating product of red blood cells similar to haem), a low temperature $\left(28^{\circ} \mathrm{C}\right)$, and possible nutrient starvation (between invertebrate blood meals). Stresses in the circulatory system for Bartonella include antibiotics (genotoxic or toxic by other mechanisms), oxidative stresses (from reactive oxygen species generated by neutrophils and macrophages), and other host defences, such as antimicrobial peptides or nutrient deprivation (nutritional immunity). Stresses in host intracellular niches include oxidative stress (from reactive oxygen species), and other cellular defences such as the low $\mathrm{pH}$ of lysosomes. 
When bacteria experience exposure to an environmental stress, a global change in bacterial transcription takes place to adapt to that environment. Alterations to cellular processes and metabolism simultaneously slow growth and unnecessary functions while promoting survival and nutrient uptake. This process is driven by various pathways, known collectively as bacterial stress response pathways ${ }^{[179-181]}$. Though a specific stress may activate a specific stress response pathway, i.e., a high temperature will activate the heat shock response, bacterial stress response pathways have extensive crosstalk and work in conjunction to respond to stresses. Stress adaptation is controlled by sigma factors ( $\sigma$ factors) that modulate bacterial expression to promote bacterial survival and downregulate non-essential systems. The components of stress pathways are essential in regular cellular functions such as growth, division, transcription, and virulence ${ }^{[182-}$ ${ }^{184]}$. The best studied Bartonella stress response pathways are the general stress response and the stringent response pathways.

\subsubsection{Bartonella General Stress Response}

The general stress response protects Alphaproteobacteria (e.g. B. henselae), Gammaproteobacteria (e.g. E. coli), and Firmicutes (e.g. Bacillus subtilis) against non-specific stresses, such as oxidative stress, heat and $\mathrm{pH}$, and provides cross resistance to multiple stresses (carbon-starved gammaproteobacteria cells gain resistance to $\mathrm{H}_{2} \mathrm{O}_{2}$, low $\mathrm{pH}$, and high temperatures) ${ }^{[185]}$. The general stress response is present in several bacterial lineages, although the mechanisms vary between phylum and class of bacteria.

In $B$. henselae, the general stress response is regulated through the extracytoplasmic function (ECF) sigma factor $\left(\sigma^{\mathrm{EcfG}}\right)$ partner switching mechanism (see Figure 1.2 below). When stress is low, $\sigma^{\mathrm{EcfG}}$ is inactivated by binding to an anti-sigma 
factor, NepR. When a histidine kinase sensor detects an environmental stress, the sensor undergoes an autophosphorylation reaction, subsequently transferring a phosphoryl group from to PhyR. Phosphorylated PhyR interacts with NepR, releasing the $\sigma^{\text {EcfG }}$ sigma factor and enabling it to interact with RNA polymerase enzyme to promote stressrelated transcription.

a.

Histidine Kinase Detects Stress and Activates Autophosphorylation

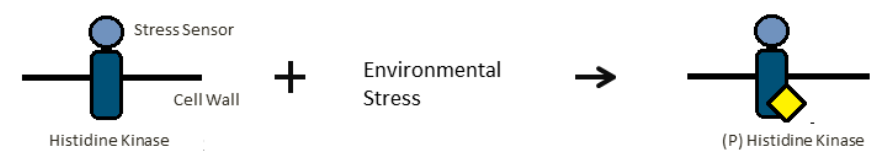

b.

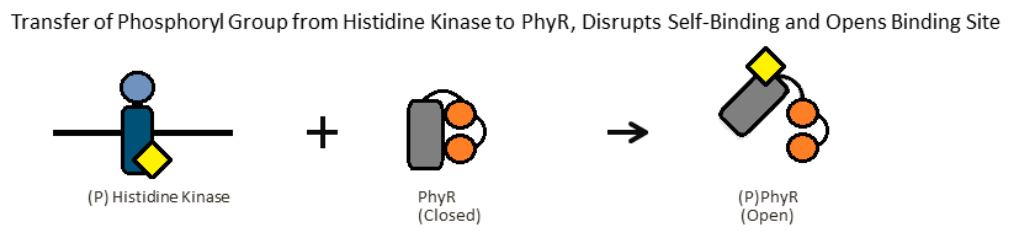

c.

Phosphorylated PhyR Binds to NepR, Disrupts NepR- $\sigma^{\mathrm{EcfG}}$ Binding and Frees $\sigma^{\mathrm{EcfG}}$ Sigma Factor

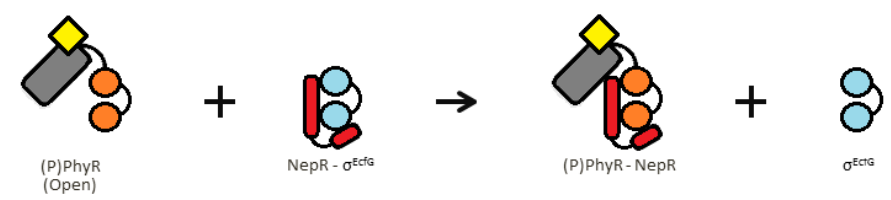

Figure 1.2. - Bartonella general stress response partner switching mechanism. PhyR = anti-anti-sigma factor, NepR = anti-sigma factor, $\sigma^{\text {EcfG }}=$ EcfG Sigma factor ${ }^{[186]}$. a) An environmental stress external to the bacterial cell activates the histidine kinase sensor embedded in the cell wall, leading to autophosphorylation of the histidine kinase. b) The phosphoryl group of histidine kinase is transferred to the inactive PhyR, opening the binding site. c) The open binding site of the phosphorylated PhyR group disrupts binding of NepR- $\sigma^{\mathrm{EcfG}}$, freeing $\sigma^{\mathrm{EcfG}}$ to interact with RNA polymerase to activate a transcriptional response.

\subsubsection{Bartonella Stringent Response}

The stringent response is a process which is ubiquitous in bacteria, but also occurs on several plant species, and in some higher organisms ${ }^{[187]}$. Originally thought to solely be a response to amino acid nutrient deprivation, the stringent response has been found to 
be critical for the cellular response to various stresses in E. coli and other bacteria. Major cellular processes that are affected by the stringent response include cellular growth, adaptation to new conditions, survival, persistence, cellular division, and virulence ${ }^{[188-}$ 193]. The stringent response plays an important role in defence against several classes of antibiotics, including fluoroquinolones ${ }^{[190,194]}$. Inhibiting the stringent response lowers bacterial resistance to hydrogen peroxide, a common reactive oxygen species (ROS) generated by the respiratory burst of macrophages and neutrophils ${ }^{[195-197]}$.

The stringent response is characterised by the presence of (p)ppGpp alarmones, the collective name of the GDP and GTP analogues guanosine tetraphosphate (ppGpp) and guanosine pentaphosphate (pppGpp ${ }^{[198,199]}$. (p)ppGpp is important in regulating normal cellular division, growth, virulence, and DNA replication, among other pathways ${ }^{[182,184]}$. In the stringent response, (p)ppGpp levels regulate transcription and metabolism. This can be done through direct binding to RNA polymerase ${ }^{[200]}$, by interacting with $\sigma$ factors (indirectly via assisting in alternate $\sigma$ factor binding to RNA polymerase or by directly via binding to multiple $\sigma$ factors $)^{[201-203]}$, or by promoting GTP and amino acid uptake and production ${ }^{[204,205]}$. Accumulation of cellular (p)ppGpp promotes survival, while low concentration of (p)ppGpp promotes cellular growth ${ }^{[206]}$. Levels of (p)ppGpp are regulated by RelA/SpoT homologues and small alarmone synthetase or hydrolase proteins; all bacteria have a combination of (p)ppGpp regulatory genes. E. coli encodes the two originally identified relA and spoT genes, from which relA/spoT homologues are named. RelA synthesises (p)ppGpp from GTP, GDP or ATP, while SpoT can either synthesise or hydrolyse (p)ppGpp into the base components of GDP and pyrophosphate $\left(\mathrm{P}_{\mathrm{i}}\right)$ or GTP and $\mathrm{PP}$. 
In Bartonella, the stringent response is controlled by DksA and SpoT, which regulate the two component system BatR/Bat $S^{[207]}$, and the alternate sigma factor RpoH1. Interestingly, in $B$. henselae both VirB/D4 and Trw T4SSs are regulated by the bacterial stringent response (see Figure $\mathbf{1 . 3}^{[207,208]}$. The stringent response is hypothesised to detect small environmental changes in stress (e.g. nutrients and $\mathrm{pH}$ ) to modulate bacterial virulence in early and late Bartonella infection - in nutrient starvation the stringent response triggers up-regulation of VirB/D4 T4SS mediated invasion of the primary niche, while Trw T4SS is down-regulated. However, in the nutrient rich bloodstream, down-regulation of the stringent components SpoT and DksA and the upregulation of BatR activates the Trw T4SS to mediate invasion of mammalian erythrocytes.

Additionally DksA in Bartonella, E. coli, and other Gram-negative bacteria (commonly in alpha- and gamma-proteobacteria) assists in stabilising the (p)ppGpp-RNA polymerase complex by binding to the secondary channel of RNA polymerase ${ }^{[209,210]}$. DksA in E. coli plays a role in stimulating the stringent response, by potentiating (p)ppGpp activity via binding to RNA polymerase ${ }^{[211]}$. In Mycobacteria, the role of DksA in the stringent response appears to be carried out by a CarD homologue $\operatorname{protein}^{[212]}$. Despite a lack of CarD sequence homology to DksA, M. smegmatis CarD could functionally replace DksA in E. coli, although the reverse was not true. While both bind to RNA polymerase, DksA binds to the secondary channel of the complex, while CarD binds to the $\beta$-subunit. Interestingly, Bartonella species have both CarD and DksA proteins. 


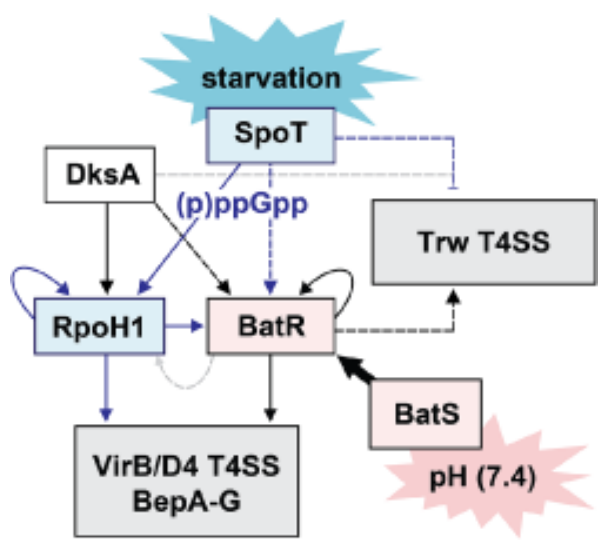

Figure 1.3. - Hypothetical B. henselae stringent response control over the VirB/D4 and Trw type 4 secretion systems (T4SS) Unbroken arrows denote positive effect, dashed lines possible indirect effects (i.e. through other proteins such as KorA/KorB). Figure has been reproduced from: Molecular Microbiology, 90/4, Québatte, et al, Dual input control: activation of the Bartonella henselae VirB/D4 type IV secretion system by the stringent sigma factor RpoH1 and the BatR/BatS two-component system, pages 756-75, 2013, with permission from John Wiley and Sons ${ }^{[207]}$.

\subsection{CarD}

The CarD protein was first described in Myxococcus xanthus, where the protein acts as a global transcriptional regulator, through binding with RNA polymerase and DNA. The protein regulates several cellular functions, such as carotenoid synthesis and formation of fruiting bodies during starvation ${ }^{[213,214]}$. However, the CarD protein reported in the literature for Mycobacterium and Thermus genera, along with the Bartonella CarD protein that is the focus of this study, are actually homologues of the M. xanthus CdnL (CarD N-terminal Like protein), rather than M. xanthus CarD. Though both M. xanthus CarD and CdnL act as transcriptional regulators, and have a high degree of structural protein homology (a conserved Tudor-like structure at the N-terminal domain), they have divergent functions; while CarD deletion inhibits $M$. xanthus growth, a CdnL deletion is lethal ${ }^{[215-217]}$.

In Mycobacterium, a genus that includes M. tuberculosis, a human pathogen that is the causative agent of tuberculosis, the $\operatorname{CarD}(M$. xanthus $\mathrm{CdnL})$ homologue is essential ${ }^{[212]}$. CarD from M. tuberculosis directly binds to RNA polymerase and DNA to regulate 
rRNA transcription during both regular bacterial function and in response to environmental stresses ${ }^{[212,218]}$. During transcription, the RNA polymerase holoenzyme binds to a promoter site, unwinding the DNA to form the open promoter complex ${ }^{[216]}$, a process in which CarD assists in several ways. The CarD N-terminal domain binds to the $\beta$-subunit of RNA polymerase to stabilise the RNA polymerase-promoter complex ${ }^{[219]}$. In addition, the CarD C-terminal domain binds to a site upstream of the promoter site, revealed by the open promoter complex, to prevent collapse of the transcription bubble ${ }^{[220,221]}$. Through these interactions with RNA polymerase and DNA, CarD regulates expression of a wide array of genes and as such can be labelled a global regulator ${ }^{[212,222]}$. Interestingly, although $M$. xanthus $\mathrm{CdnL}$ cannot bind to DNA, it is still involved in stabilisation of the open promoter complex, through its interaction with RNA polymerase ${ }^{[215]}$.

Among other bacterial processes, Mycobacterium CarD is involved in the bacterial response to stress, through the stringent response pathway. Transient deletion of $\operatorname{carD}$ in $M$. smegmatis lead to increased sensitivity to ciprofloxacin, hydrogen peroxide and starvation $^{[212,223]}$. In M. smegmatis cultures exposed to starvation and $\mathrm{H}_{2} \mathrm{O}_{2}$ conditions, an accumulation of (p)ppGpp and a down-regulation of rRNA was observed. However, when expression of $c a r D$ was artificially lowered, in cultures exposed to starvation and $\mathrm{H}_{2} \mathrm{O}_{2}$ conditions, a reduced accumulation of (p)ppGpp was observed, while rRNA levels observed were the same as for unstressed cells ${ }^{[212]}$.

As mentioned previously, CarD in Mycobacterium appears to carry out the same function as E. coli DksA in the bacterial stringent response ${ }^{[211,212]}$. This is especially interesting, considering that Bartonella species have both CarD and DksA proteins ${ }^{[207]}$, although as of yet, the functionality of CarD has not been described. In contrast to 
M. tuberculosis, little is known about the structure or function of CarD in Bartonella species, although bioinformatic analysis of the gene indicates that it likely binds RNA polymerase similar to other bacterial species (for protein sequence comparison alignments see Appendix Figure 8.1). Similarly to E. coli DksA, neither B. henselae CarD nor DksA appear to be essential genes for growth, in contrast with several other bacterial CarD homologues ${ }^{[215,216,224]}$. Deletion has a negative effect on bacterial pathways $^{[176,207]}$, as $\Delta c a r D B$. birtlesii lacked the ability to establish bacteraemia in a mouse, and $\triangle d k s A B$. henselae mutants had reduced in vitro invasome formation during endothelial cell invasion, through disruption of the VirB/D4 T4SS system ${ }^{[207]}$. This differs from $M$. xanthus, where both $\operatorname{carD}$ and $d k s A$ were shown to be essential genes $^{[225]}$

\subsection{Research Aims}

The overall aim of this thesis was to elucidate the role of the CarD homologue in the B. henselae response to common environmental stresses. To this end we undertook two strategies to investigate the activity of $B$. henselae carD. Our first aim was to create a $\Delta c a r D$ mutant strain of $B$. henselae. This involved inducing homologous recombination of our wild-type $B$. henselae and mutagenic plasmids containing sequence of $B$. henselae $\operatorname{carD}$ gene; the result of this would be to knock out function of the $\operatorname{carD}$ gene. Wildtype and $\Delta c a r D$ mutant strains of $B$. henselae would be subjected to environmental stresses, then growth measured to determine the effect of $\operatorname{carD}$ deletion on $B$. henselae growth. Despite multiple attempts to generate a $\Delta c a r D$ strain, a mutant was not obtained. The second aim was to carry out transcriptional analysis of $B$. henselae carD expression during exposure to environmental stresses. Before this aim could be achieved, a series of stress response assays were carried out on $B$. henselae colonies to determine the 
appropriate stress conditions to be used in our quantitative PCR experiments. The overall goal of this thesis was to determine if the B. henselae carD gene behaved in a similar manner to $\operatorname{car} D$ genes in other well-studied bacterial models. 


\section{Chapter 2. Methods}

\subsection{Oligonucleotide Primers}

All primers were synthesised and lyophilised for shipment by Integrated DNA Technologies (IDT; Singapore). Primers were resuspended at a concentration of $100 \mu \mathrm{M}$ in autoclaved $0.22 \mu \mathrm{m}$ filter-sterilized $\mathrm{ddH}_{2} \mathrm{O}$, and stored at $-20^{\circ} \mathrm{C}$. Working stocks were diluted to $10 \mu \mathrm{M}$ in autoclaved filter-sterilized $\mathrm{ddH}_{2} \mathrm{O}$, and stored at $-20^{\circ} \mathrm{C}$. Primers are listed by reaction pair in Table 2.1 .

Primers prJS01 forward and prJS02 reverse were designed with engineered BamHI restriction enzyme sites at the 5' end (Table 2.1, underlined), and prJS02 reverse 2 was designed with an engineered SalI restriction site (underlined). Primers prJS01 reverse and prJS02 forward were designed with complementary sequences to enable internal priming and generation of a PCR fusion product (Table 2.1, bold).

Table 2.1. $\quad$ - Oligonucleotide Primers Underlined primer sequences denote restriction sites. Bold sequences denote overlapping nucleotides. * prJS02 reverse 2 primer is a duplicate of the original prJS02 reverse primer, albeit with a BamHI restriction site instead of a Sall site.

\begin{tabular}{|c|c|c|}
\hline Primer Name & Primer Sequence & Primer Target/Function \\
\hline \multicolumn{3}{|l|}{ prJSO1 } \\
\hline prJS01_Fwd & 5' - GCGGGATCCCCTGCAGAAGCCATTAAACC - 3' & B. henselae carD 3' \\
\hline prJSO1_Rev & 5' - TGGGAGAGATGTCGCAAAATGAATGACGAAAT - 3' & Flanking Region \\
\hline \multicolumn{3}{|l|}{ prJSO2 } \\
\hline prJSO2_Fwd & 5' - TCATTTTGCGACATCTCTCCCAAAAGCCAAA - 3' & B. henselae carD 5' \\
\hline prJSO2_Rev & 5' - GCGGTCGACCGTTCAGTGTTGCCTGCT - 3' & Flanking Region \\
\hline prJSO2_Rev2* & 5' - GCGGATCCCGTTCAGTGTTGCCTGCTAA - 3' & \\
\hline \multicolumn{3}{|l|}{ prJSO3 } \\
\hline 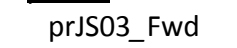 & 5' - TGCGCAAATATCTGCTGTC - 3' & B. henselae carD primer for \\
\hline prJSO3_Rev & 5' - TTCGTTAACTGCGCTTTT - 3' & qPCR \\
\hline \multicolumn{3}{|l|}{ prJSO4 } \\
\hline$\overline{p r J S 04}$ _Fwd & 5' - TCCGTGGGTGCTTGATACA - 3' & B. henselae rpsL \\
\hline prJSO4_Rev & 5' - GCTTCGCGCCATACTTGA - 3' & primer for qPCR \\
\hline \multicolumn{3}{|l|}{ M13 } \\
\hline M13_Fwd & $5^{\prime}$ - GTAAAACGACGGCCAG - 3' & Universal Sequencing Primers \\
\hline M13_Rev & 5' - CAGGAAACAGCTATGAC - 3' & \\
\hline
\end{tabular}




\subsection{Bacterial Strains}

All bacterial strains used in this study were suspended in glycerol freezer stocks and stored at $-80^{\circ} \mathrm{C}$. Strains are listed by species in Table 2.2 .

The B. henselae Houston-1 strain was streaked onto Columbia blood agar (CBA) by the ESR Culture Collection, and streaked and grown on CBA or chocolate agar plates. Confluent $B$. henselae plates were harvested and resuspended in tryptic soy broth containing $20 \%$ glycerol and stored at $-80^{\circ} \mathrm{C}$. S17-1 E. coli cells were transported in gel plugs by BioMedal Life Sciences (Spain), and used to inoculate fresh LB media. S17-1 cells were harvested and resuspended in E. coli glycerol stocks (LB with $20 \%$ glycerol) and stored at $-80.0^{\circ} \mathrm{C}$.

Table 2.2. - Strains of Bacteria

\begin{tabular}{|c|c|c|c|}
\hline Strain & Characteristics & Source & \\
\hline \multicolumn{4}{|l|}{ B. henselae } \\
\hline Houston-1 ${ }^{[85]}$ & $\begin{array}{l}\text { Wild type, Cefazolin, Nalidixic acid, and } \\
\text { sucrose resistant, Kanamycin and } \\
\text { Gentamicin sensitive }\end{array}$ & $\begin{array}{l}\text { New Zealand Reference } \\
\text { Collection (ESR) }\end{array}$ & Culture \\
\hline \multicolumn{4}{|l|}{ E. coli } \\
\hline DH10B & $\begin{array}{l}\text { High competency (electrocompetent) } \\
\text { cloning strain }\end{array}$ & Life Technologies & \\
\hline $\begin{array}{l}\text { DH10B } \\
\text { pEX18gm plasmid }\end{array}$ & $\begin{array}{l}\text { DH10B containing pEX18gm plasmid, } \\
\text { Gentamicin resistant }\end{array}$ & This study & \\
\hline $\begin{array}{l}\text { DH10B } \\
\text { pJM05 plasmid }\end{array}$ & $\begin{array}{l}\text { DH10B containing pJM05 plasmid, } \\
\text { Kanamycin resistant }\end{array}$ & pJM05 from Dr Koehler, UCSF & \\
\hline $\begin{array}{l}\text { DH10B } \\
\text { pJS01 plasmid }\end{array}$ & $\begin{array}{l}\text { DH10B containing pJS01 plasmid, } \\
\text { Gentamicin resistant, sucrose sensitive }\end{array}$ & This study & \\
\hline $\begin{array}{l}\mathrm{DH} 10 \mathrm{~B} \\
\text { pJS02 plasmid }\end{array}$ & $\begin{array}{l}\text { DH10B containing pJS02 plasmid, } \\
\text { Kanamycin resistant, sucrose sensitive }\end{array}$ & This study & \\
\hline S17-1 & $\begin{array}{l}\text { E. coli strain for biparental conjugations, } \\
\lambda \text { pir lysogen }\end{array}$ & BioMedal Life Science (Spain) & \\
\hline $\begin{array}{l}\text { S17-1 } \\
\text { pJS01 plasmid }\end{array}$ & E. coli S17-1 containing pJS01 plasmid & This study & \\
\hline $\begin{array}{l}\text { S17-1 } \\
\text { pJS02 plasmid }\end{array}$ & E. coli S17-1 containing pJS02 plasmid & This study & \\
\hline
\end{tabular}




\subsection{Plasmids}

All plasmids used in this study were suspended either in autoclaved $0.22 \mu \mathrm{m}$ filter-sterilized $\mathrm{ddH}_{2} \mathrm{O}$ or elution buffer $(10 \mathrm{mM}$ Tris- $\mathrm{HCl})$, and stored at $-20^{\circ} \mathrm{C}$. Plasmids are listed in Table 2.3. The plasmid supplied by Dr Jane Koehler (UCSF) was lyophilised for transportation, then resuspended in $0.22 \mu \mathrm{m}$ filter-sterilized $\mathrm{ddH}_{2} \mathrm{O}$ and stored at $-20^{\circ} \mathrm{C}$.

Table 2.3. - Bacterial Plasmids

\begin{tabular}{|c|c|c|c|}
\hline Plasmid & Characteristics & Source & Size (bp) \\
\hline pEX18gm & $\begin{array}{l}\text { Gentamicin resistant, oriT (origin of } \\
\text { transfer), sacB (sucrose sensitive), } \\
\text { derived from } \mathrm{pEX18ap}\end{array}$ & $\begin{array}{l}\text { Hoang et al[226], kindly } \\
\text { provided by Dr David } \\
\text { Ackerley (VUW) }\end{array}$ & $\sim 5,830$ \\
\hline $\mathrm{pJS} 01$ & $\begin{array}{l}\text { Mutagenic plasmid with carD flanking } \\
\text { sequence, cloned into pEX18gm }\end{array}$ & This study & $\sim 6730$ \\
\hline pJM05 & $\begin{array}{l}\text { Kanamycin resistant, oriT, sacB, derived } \\
\text { from pRS14[227] }\end{array}$ & $\begin{array}{l}\text { MacKichan et al[228], } \\
\text { kindly provided by Dr } \\
\text { Jane Koehler (UCSF) }\end{array}$ & $\sim 8,600$ \\
\hline $\mathrm{pJSO2}$ & $\begin{array}{l}\text { Mutagenic plasmid with carD flanking } \\
\text { sequence, cloned into pJM05 }\end{array}$ & This study & $\sim 9,910$ \\
\hline
\end{tabular}

\subsection{Growth Media}

\subsubsection{Liquid Media}

\section{Lysogeny Broth, LB (1 L) -}

Premixed powder (20 g; Duchefa Biochemie) was mixed in distilled water $(1 \mathrm{~L})$, and then autoclaved for 15 minutes at $120^{\circ} \mathrm{C}$. Media was stored for up to two months at room temperature. 


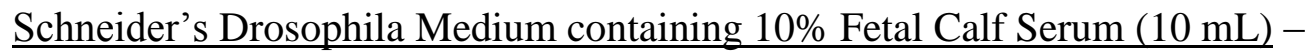

Schneider's Drosophila Medium (9 mL; Gibco $\left.^{\text {TM}}\right)$ was supplemented with Fetal Calf Serum (FCS, $1 \mathrm{~mL}$ ) inside a biosafety cabinet, and then used immediately.

$\underline{\text { M199S medium (10 mL) - }}$

M199 medium (8 $\mathrm{mL} ;$ Gibco $\left.^{\mathrm{TM}}\right)$ was supplemented with GlutaMAX $\left(100 \mu \mathrm{L}\right.$ of $200 \mathrm{mM}$; Gibco $\left.{ }^{\mathrm{TM}}\right)$, Sodium Pyruvate $\left(100 \mu \mathrm{L}\right.$ of $100 \mathrm{mM}$; Gibco $\left.{ }^{\mathrm{TM}}\right)$, and FCS ( $2 \mathrm{~mL})$ inside a biosafety cabinet, sterilised by $0.22 \mu \mathrm{m}$ filter and then used immediately.

$\underline{\text { SOC Media (1 L) - }}$

Tryptone $(20 \mathrm{~g})$, yeast extract $(5 \mathrm{~g}), \mathrm{NaCl}(2 \mathrm{~mL}$ of $5 \mathrm{M}), \mathrm{KCl}(2.5 \mathrm{~mL}$ of $5 \mathrm{M})$ were mixed in distilled water $(1 \mathrm{~L})$, and then autoclaved for 15 minutes at $120^{\circ} \mathrm{C}$. Media was cooled to $50^{\circ} \mathrm{C}$, and then supplemented with sterile, autoclaved $\mathrm{MgCl}_{2}(10 \mathrm{~mL}$ of $1 \mathrm{M})$ and $0.22 \mu \mathrm{m}$ filter-sterilized glucose $(20 \mathrm{~mL}$ of $1 \mathrm{M})$. Media was stored up to two months at $4^{\circ} \mathrm{C}$.

\section{E. coli Freezer stock $(50 \mathrm{~mL})-$}

LB $(25 \mathrm{~mL})$ and glycerol $(25 \mathrm{~mL}$ of $80 \%)$ were combined and then autoclaved for 15 minutes at $120^{\circ} \mathrm{C}$. Media was cooled and stored up to a month.

\section{B. henselae Freezer stock (50 mL) -}

Tryptic Soy Broth $(40 \mathrm{~mL})$ and glycerol $(10 \mathrm{~mL}$ of $80 \%)$ were combined and autoclaved for 15 minutes at $120^{\circ} \mathrm{C}$. Media was cooled and used immediately.

\subsubsection{Solid Media}

\section{$\underline{\text { LB agar plates }}-$}

Premixed LB agar powder (32 g; Life Technologies) was mixed in distilled water (1 L), and then autoclaved for 15 minutes at $120^{\circ} \mathrm{C}$. Supplements were added when molten 
media was cooled to $50^{\circ} \mathrm{C}$. Media was pipetted $(20 \mathrm{~mL})$ into standard $90 \mathrm{~mm}$ petri dishes in a sterile biosafety cabinet. LB plates were cooled at room temperature until the agar solidified, at which point they were stored at for two months at $4^{\circ} \mathrm{C}$ or one month if supplemented with antibiotics.

\section{$\underline{\text { Chocolate agar plates }}^{[229]}$}

GC agar solution (36 g of GC agar powder, in $500 \mathrm{~mL}$ distilled water; Thermo Fisher Scientific) and haemoglobin solution (10 $\mathrm{g}$ of $2 \%$ freeze-dried bovine haemoglobin powder, in $500 \mathrm{~mL}$ distilled water; BD BBL) were prepared separately by gentle mixing. Solutions were gently heated $\left(\sim 60\right.$ to $\left.70^{\circ} \mathrm{C}\right)$ on a magnetic stirring plate, and were then autoclaved for 15 minutes at $120^{\circ} \mathrm{C}$. Solutions were cooled to $50^{\circ} \mathrm{C}$, and then combined along with IsoVitaleX ${ }^{\mathrm{TM}}$ media enrichment $(10 \mathrm{~mL}$; Becton Dickinson) and any other supplements in a Class II bio-safety cabinet. Media was carefully pipetted ( $27 \mathrm{~mL})$ into $90 \mathrm{~mm}$ petri dishes, cooled at room temperature and then stored for one month at $4^{\circ} \mathrm{C}$.

\subsubsection{Media Supplements}

All media supplements or antibiotics were made up to the noted stock concentration in the appropriate solvent and stored at $-20^{\circ} \mathrm{C}$, with the exceptions of haemin $\left(4^{\circ} \mathrm{C}\right)$, hydrogen peroxide (room temperature), and sucrose (used immediately). Media supplements are listed in Table 2.4 .

Ciprofloxacin was suspended in dilute $\mathrm{HCl}(25 \mathrm{mg} / \mathrm{mL}$ in $0.1 \mathrm{M})$ then diluted to desired stock concentration and stored at $-20^{\circ} \mathrm{C}$. Haemin was prepared as described by Roden et al ${ }^{[230]}$, and stored for one week at $4^{\circ} \mathrm{C}$. Sucrose was resuspended in $0.22 \mu \mathrm{L}$ filter-sterilized $\mathrm{ddH}_{2} \mathrm{O}$, and used immediately. 
Table 2.4. - Media Supplements and Concentrations E.C denotes E. coli, while B. $h$ denotes B. henselae

\begin{tabular}{llll}
\hline Supplement & Media & Stock Concentration & $\begin{array}{l}\text { Media } \\
\text { Concentration }\end{array}$ \\
\hline \hline Ciprofloxacin & Schneider's Medium & $10 \mathrm{mg} / \mathrm{mL}$ & $10,25 \mu \mathrm{g} / \mathrm{mL}$ \\
Haemin & Schneider's Medium & $1 \mathrm{mM}$ & $0.1,0.5 \mathrm{mM}$ \\
Hydrogen Peroxide $\left(\mathrm{H}_{2} \mathrm{O}_{2}\right)$ & Schneider's Medium & $36 \% \mathrm{w} / \mathrm{v}$ & $0.1,1 \mathrm{mM}$ \\
Kanamycin & LB medium, LB agar & $50 \mathrm{mg} / \mathrm{mL}(E . c)$ & $50 \mu \mathrm{g} / \mathrm{mL}(E . c)$ \\
& plates, Chocolate agar & $30 \mathrm{mg} / \mathrm{mL}(B . h)$ & $30 \mu \mathrm{g} / \mathrm{mL}(B . h)$ \\
Gentamicin & LB medium, LB agar & $10 \mathrm{mg} / \mathrm{mL}$ & $10 \mu \mathrm{g} / \mathrm{mL}$ \\
Pefazolin & plates, Chocolate agar & & $20 \mu \mathrm{g} / \mathrm{mL}$ \\
Nalidixic acid & Chocolate agar & $50 \mathrm{mg} / \mathrm{mL}$ & $2 \mu \mathrm{g} / \mathrm{mL}$ \\
Sucrose & Chocolate agar & $10 \mathrm{mg} / \mathrm{mL}$ & $10 \% \mathrm{Sucrose}$ \\
\hline
\end{tabular}

\subsection{Culture Conditions of Bacteria}

\section{B. henselae on Chocolate Agar Plates -}

For confluent plates, $-80^{\circ} \mathrm{C}$ glycerol stocks of $B$. henselae were streaked onto plain chocolate agar plates in a biosafety cabinet. Plates were incubated for seven to 10 days in a humidified $5 \% \mathrm{CO}_{2}, 37^{\circ} \mathrm{C}$ stationary incubator. $B$. henselae were harvested and re-streaked onto fresh chocolate agar plates, and then incubated for five to seven days to generate confluent $B$. henselae plates. Confluent plates were passaged several times before being replaced with fresh $-80^{\circ} \mathrm{C} B$. henselae glycerol stocks.

\section{E. coli on LB Plates and in LB Broth -}

E. coli was streaked onto plain LB agar plates from $-80^{\circ} \mathrm{C}$ glycerol stocks in a biosafety cabinet, and then incubated overnight in a $37^{\circ} \mathrm{C}$ stationary incubator. Fresh LB broth $(10 \mathrm{~mL})$ in a $50 \mathrm{~mL}$ Falcon tube was inoculated with a single colony, and then grown overnight in a $37^{\circ} \mathrm{C}$ rotary incubator. Overnight culture was recovered in the morning; fresh LB media $(9 \mathrm{~mL})$ was inoculated with overnight culture $(1 \mathrm{~mL})$, and then incubated for $\sim$ three hours at $37^{\circ} \mathrm{C}$ in a rotary incubator until the $\mathrm{OD}_{600}$ reached 1.0. The 
$\mathrm{OD}_{600}$ (optical density of liquid at a wavelength of $600 \mathrm{~nm}$ ) was measured in 10x 10x45 mm clear plastic cuvettes using a Nicolet Evolution 100 UV-Vis Spectrophotometer (Thermo Scientific).

\section{Preparation of E. coli Electrocompetent Cells}

An E. coli liquid culture $(10 \mathrm{~mL})$ was prepared from a $-80^{\circ} \mathrm{C}$ Glycerol stock as per above then incubated overnight. The culture was diluted 1:10 and then incubated for several hours in a $37^{\circ} \mathrm{C}$ rotary incubator, during which the growth rate was monitored until the $\mathrm{OD}_{600}$ reached 0.35 to 0.4 ; the culture was removed from the incubator and cooled for 15 to 20 minutes on ice. Chilled cultures were transferred to pre-chilled Falcon tubes and centrifuged for 10 minutes at $3,000 \times g$, at $4{ }^{\circ} \mathrm{C}$. The supernatant was poured off and ice-cold $25 \%$ glycerol ( 25 to $50 \mathrm{~mL}$ ) added. The Falcon tubes were gently rotated and inverted to remove latent salts from the walls of the tube, taking care not to disturb the pellet. The suspensions were washed via centrifugation a further three times in ice-cold $25 \%$ glycerol $(50 \mathrm{~mL})$ for 10 minutes at $3,000 \times \mathrm{g}$, at $4^{\circ} \mathrm{C}$. After each spin, the supernatant was poured off, fresh glycerol was added, and the tubes rotated and inverted as before. After the final centrifugation step, the supernatant was poured off and the pellet resuspended in glycerol $(40 \%)$ as a freezer stock $(5 \mathrm{~mL})$. Aliquots of electrocompetent $E$. coli cultures $(100 \mu \mathrm{L})$ were divided into cryovials and either used immediately or stored at $-80^{\circ} \mathrm{C}$.

\section{Preparation of $B$. henselae Electrocompetent Cells}

Preparation of electrocompetent $B$. henselae cells was carried out essentially as described in Riess et al, with several modifications ${ }^{[231]}$. Two confluent $B$. henselae chocolate agar plates were harvested and then resuspended in ice-cold M199S medium $(10 \mathrm{~mL})$ in a $50 \mathrm{~mL}$ Falcon tube and cooled alongside autoclaved $10 \%$ glycerol for 
10 to 15 minutes on ice. Cultures were then centrifuged for six minutes at 3,000 x g, at $4^{\circ} \mathrm{C}$. The supernatant was removed and pellets were resuspended in ice-cold $10 \%$ glycerol $(5 \mathrm{~mL})$. Cultures were washed via centrifugation a further three times in ice-cold $10 \%$ glycerol (5 mL). After each spin, the supernatant was poured off, and the pellets were resuspended in fresh $10 \%$ glycerol. After the final spin, supernatant was poured off and the pellet resuspended in $10 \%$ glycerol. Aliquots of electrocompetent B. henselae cultures $(100 \mu \mathrm{L})$ were used immediately.

\subsection{Molecular Biology Protocols}

\subsubsection{Isolation of Genomic DNA}

Bacterial genomic DNA was purified using the CTAB extraction method ${ }^{[232]}$. Briefly, B. henselae was scraped from plates and suspended in TE buffer $(567 \mu \mathrm{l} ; 10 \mathrm{mM}$ Tris- $\mathrm{HCl}, 1 \mathrm{mM}$ EDTA, $\mathrm{pH} 8.0)$ in a $1.5 \mathrm{~mL}$ microcentrifuge tube. Proteinase $\mathrm{K}(3 \mu \mathrm{l}$ of $20 \mathrm{mg} / \mathrm{mL}$ ) and SDS (30 $\mu \mathrm{L}$ of $10 \%)$ were added, and the suspension was incubated for one hour in a $37^{\circ} \mathrm{C}$ stationary incubator. $\mathrm{Next}, \mathrm{NaCl}(100 \mu \mathrm{l}$ of $5 \mathrm{M})$ was added to the suspension and mixed thoroughly, before CTAB-NaCl $(80 \mu \mathrm{l} ; 10 \% \mathrm{CTAB}$ [hexadecyltrimethyl ammonium bromide] in $0.7 \mathrm{M} \mathrm{NaCl}$ ) was added. The suspension was incubated for 10 minutes at $65^{\circ} \mathrm{C}$ on a heating block. An equivalent volume ( $\sim .7$ to $0.8 \mathrm{ml}$ ) of chloroform:isoamyl alcohol (24:1) was added, vortexed, then centrifuged for five minutes at $10,000 \times g$. After centrifugation, the upper, aqueous phase was removed with a narrow pipette and transferred to a fresh $1.5 \mathrm{~mL}$ microcentrifuge tube. An equivalent volume of phenol:chloroform:isoamyl alcohol (25:24:1) was added, vortexed, and centrifuged for five minutes at $10,000 \times \mathrm{x}$. Isopropanol (0.6 volume) was added, then the tube was inverted gently until a visible DNA precipitate formed. The 
DNA was pelleted by centrifugation and then washed with ethanol $(500 \mu \mathrm{L}$ of $70 \%$ EtOH). The tube was gently inverted to mix the solution, and then centrifuged for five minutes at $10,000 \times g$. The supernatant was removed and the pellet was air dried. The pellet was then resuspended in TE buffer $(100 \mu \mathrm{l})$ for freezer storage at $-20^{\circ} \mathrm{C}$.

\subsubsection{Polymerase Chain Reaction (PCR)}

All standard PCR reactions in this study were performed using either Advantage ${ }^{\circledR} 2$ Polymerase (Clontech) or with Phusion ${ }^{\circledR}$ High-Fidelity PCR Polymerase Mastermix (New England Biolabs) in $0.2 \mathrm{~mL}$ PCR tubes. Components and quantities of each PCR are listed in Table 2.5 and PCR conditions in Table 2.6.

Table 2.5. - Standard PCR Components and Quantities

\begin{tabular}{ll}
\hline PCR Components & Quantity \\
\hline \hline Advantage Reaction Mixture & \\
\hline Forward Primer $(10 \mu \mathrm{M})$ & $1 \mu \mathrm{L}(200 \mathrm{nM})$ \\
Reverse Primer $(10 \mu \mathrm{M})$ & $1 \mu \mathrm{L}(200 \mathrm{nM})$ \\
dNTPs $(10 \mathrm{mM}$ of each dNTP) & $1 \mu \mathrm{L}(200 \mu \mathrm{M})$ \\
Advantage Buffer $(10 \mathrm{x})$ & $5 \mu \mathrm{L}(1 \mathrm{x})$ \\
Advantage Polymerase $(50 \mathrm{x})$ & $1 \mu \mathrm{L}(1 \mathrm{x})$ \\
DNA Template & $\mathrm{x} \mu \mathrm{L}(100$ to $250 \mathrm{ng})$ \\
ddH ${ }_{2} \mathrm{O}$ & up to $50 \mu \mathrm{L}$ \\
& $\underline{50 \mu \mathrm{L} \text { Total }}$ \\
& \\
Phusion Reaction Mixture & \\
\hline Forward Primer $(10 \mu \mathrm{M})$ & $0.5 \mu \mathrm{L}(200 \mathrm{nM})$ \\
Reverse Primer $(10 \mu \mathrm{M})$ & $0.5 \mu \mathrm{L}(200 \mathrm{nM})$ \\
Phusion Master Mix $(2 \mathrm{x})$ & $12.5 \mu \mathrm{L}(1 \mathrm{x})$ \\
DNA Template & $x \mu \mathrm{L}(100$ to $250 \mathrm{ng})$ \\
ddH ${ }_{2} \mathrm{O}$ & up to $25 \mu \mathrm{L}$ \\
& $\underline{25 \mu \mathrm{L} \text { Total }}$ \\
&
\end{tabular}


Table 2.6. - Standard PCR Conditions *Extension temperature was set according to the optimum primer $\mathrm{T}_{\mathrm{m}}, 56.5^{\circ} \mathrm{C}$ for $\operatorname{carD} \mathrm{qPCR}$ primers and $62.8^{\circ} \mathrm{C}$ for $r p s L \mathrm{qPCR}$ primers, although if required both are functional at $58^{\circ} \mathrm{C}$.

\begin{tabular}{|c|c|c|c|}
\hline PCR Conditions & Temperature & Hold Time & Cycle Number \\
\hline \multicolumn{4}{|c|}{ Advantage PCR Conditions } \\
\hline $\begin{array}{l}\text { Pre-heat } \\
\quad \text { Denaturation } \\
\quad \text { Annealing } \\
\quad \text { Extension } \\
\text { Post Cycle Extension } \\
\text { Hold }\end{array}$ & $\begin{array}{l}95^{\circ} \mathrm{C} \\
95^{\circ} \mathrm{C} \\
60^{\circ} \mathrm{C} \\
68^{\circ} \mathrm{C} \\
68^{\circ} \mathrm{C} \\
4^{\circ} \mathrm{C}\end{array}$ & $\begin{array}{l}1 \text { Minute } \\
30 \text { Seconds } \\
1 \text { Minute } \\
1 \text { Minute } \\
3 \text { Minutes } \\
\text { Infinite hold }\end{array}$ & 30 cycles \\
\hline \multicolumn{4}{|c|}{$\underline{\text { Phusion PCR Conditions }}$} \\
\hline $\begin{array}{l}\text { Pre-heat } \\
\text { Denaturation } \\
\text { Annealing } \\
\text { Extension }\end{array}$ & $\begin{array}{l}98^{\circ} \mathrm{C} \\
\quad 98^{\circ} \mathrm{C} \\
56.5 \text { or } 62.8^{\circ} \mathrm{C}^{*} \\
72^{\circ} \mathrm{C}\end{array}$ & $\begin{array}{l}1 \text { Minute } \\
10 \text { Seconds } \\
20 \text { Seconds } \\
20 \text { Seconds }\end{array}$ & 30 cycles \\
\hline $\begin{array}{l}\text { Post Cycle Extension } \\
\text { Hold }\end{array}$ & $\begin{array}{l}72^{\circ} \mathrm{C} \\
4^{\circ} \mathrm{C}\end{array}$ & $\begin{array}{l}5 \text { Minutes } \\
\text { Infinite hold }\end{array}$ & \\
\hline
\end{tabular}

\subsubsection{Agarose Gel Electrophoresis}

For visualising DNA samples, DNA (5 $\mu \mathrm{L}$ with $1 \mu \mathrm{L}$ loading buffer $6 \mathrm{x})$ was run alongside a DNA reference ladder, Hyperladder ${ }^{\mathrm{TM}}$ I (5 $\mu \mathrm{L}$; Bioline), on agarose electrophoresis gels; agarose gels were $1 \% \mathrm{w} / \mathrm{v}$ of agarose in 1x TAE buffer $(100 \mathrm{~mL}$ of $40 \mathrm{mM}$ Tris-acetate, $1 \mathrm{mM}$ EDTA). Electrophoresis gels were run for 30 to 60 minutes at 100 to $120 \mathrm{~V}$ in $1 \mathrm{x}$ TAE running buffer. Agarose gels were post-stained in Gelred ${ }^{\mathrm{TM}}$ nucleic acid gel stain ( $10 \mu \mathrm{L}$ of $10,000 \mathrm{x}$ solution; Biotium) for one to two hours, and then visualised and photographed with a GelDoc (Cell Biosciences) under ultraviolet light.

\subsubsection{Isolation of Plasmids}

E. coli containing the desired plasmid was cultured overnight on selective LB media (50 $\mu \mathrm{g} / \mathrm{mL}$ Kanamycin for pJM05 or $10 \mu \mathrm{g} / \mathrm{mL}$ Gentamicin for pEX18gm or their derivatives) from either E. coli $-80^{\circ} \mathrm{C}$ glycerol stocks or from recovered E. coli 
electroporation mixtures. Plasmids were isolated from overnight cultures using a GenepHLow ${ }^{\mathrm{TM}}$ kit according to the plasmid isolation protocol, eluting in $30 \mu \mathrm{L}$ of either $0.22 \mu \mathrm{m}$ filter-sterilized $\mathrm{ddH}_{2} \mathrm{O}$, elution buffer ( $1 \mathrm{~mL}$ of $10 \mathrm{mM}$ Tris-HCl), or TE buffer (1 mL of $10 \mathrm{mM}$ Tris-HCl, $1 \mathrm{mM}$ EDTA, $\mathrm{pH}$ 8.0). Elutions were quantified ( $1 \mu \mathrm{L}$ per sample) on a NanoDrop ND-1000 ${ }^{\mathrm{TM}}$ (Thermo Fisher) machine as per manufacturer's to determine concentration. Eluted plasmids (10 to $25 \mu \mathrm{L})$ to be used for electroporation

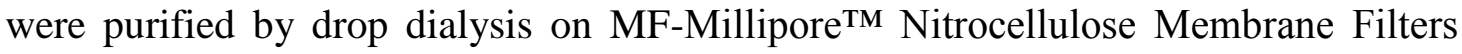
(Merck Millipore), floating on top of $\mathrm{ddH}_{2} \mathrm{O}$ for one hour. Plasmids were then carefully moved by pipette into fresh $1.5 \mathrm{~mL}$ microcentrifuge tubes and stored at $-20^{\circ} \mathrm{C}$.

\subsubsection{Purification and Quantification of DNA products}

To clean and purify plasmids and PCR products, GenepHLow ${ }^{\mathrm{TM}}$ Gel Extraction and PCR Cleanup Kit (Geneaid) or DNA Clean and Concentrator ${ }^{\mathrm{TM}}$ kit (Zymo) were used as per the manufacturer's instructions. Elutions were quantified by NanoDrop as described above and stored at $-20^{\circ} \mathrm{C}$.

\subsubsection{Size-Specific Purification of DNA products by Gel Electrophoresis}

DNA purification of size-specific PCR products was performed using gel electrophoresis and column purification. PCR products (10 to $20 \mu \mathrm{L})$ were run on an agarose gel alongside Hyperladder ${ }^{\mathrm{TM}} \mathrm{I}$ as described. Agarose gels were viewed under UV light to identify desired products, which were excised from the gel using a razorblade. Gel plugs were then heated and purified with the GenepHLow ${ }^{\mathrm{TM}}$ kit, according to the kit instructions. Elutions were quantified by NanoDrop as per above and stored at $-20^{\circ} \mathrm{C}$. 


\subsubsection{Restriction Digest and Ligation Reactions}

Restriction digests of DNA products were performed with BamHI and SalI in a double restriction digest or BamHI restriction enzymes (all Thermo Scientific). Purified and quantified DNA products were thawed, added to reaction mixture in $20 \mu \mathrm{L}$ reactions (PCR components are listed in Table 2.7) and then incubated for one hour at $37^{\circ} \mathrm{C}$ for optimal enzyme conditions. Digested products were column purified using DNA Clean and Concentrator ${ }^{\mathrm{TM}}$ kit (Zymo) to concentrate and remove salts and enzymes, and then stored at $-20^{\circ} \mathrm{C}$. Before column purification of digested pJM05, the plasmid was treated with Calf Intestinal Alkaline Phosphatase $(1 \mu \mathrm{L})$ for five minutes at $37^{\circ} \mathrm{C}$ in a stationary incubator, preventing self-ligation of plasmid BamHI ends.

All DNA ligation reactions in this study were performed using T4 DNA Ligase (New England Biolabs). Digested and purified plasmid and carD PCR Fusion insert DNA products were combined into a ligation reaction (reaction protocol is listed in Table 2.7) and incubated for one hour at room temperature. Ligated plasmid was purified by drop dialysis using Membrane Filters (Merck Millipore) for one hour to remove salts and then transformed into cloning $E$. coli strains. 
Table 2.7. - Restriction Digest and Ligation Reactions Components and Quantities DNA templates were mixed together at a molar ratio of about 1:6 (where $x 1=\mathrm{ng}$ of vector and $y 1=\mathrm{ng}$ of Insert), using the Ligation Template Ratio equation below

\begin{tabular}{|c|c|}
\hline Reaction Components & Quantity \\
\hline \multicolumn{2}{|l|}{$\underline{\text { BamHI Restriction Digest }}$} \\
\hline $\begin{array}{l}\text { BamHI Enzyme } \\
\text { NE Buffer } 3.1 \text { (10x) } \\
\text { DNA Template } \\
\text { dd }_{2} \mathrm{O}\end{array}$ & $\begin{array}{l}0.5 \mu \mathrm{L} \text { (5 units) } \\
2 \mu \mathrm{L}(1 \mathrm{x}) \\
\mathrm{x} \mu \mathrm{L}(\leq 500 \mathrm{ng}) \\
\text { up to } 20 \mu \mathrm{L} \\
20 \mu \mathrm{L} \text { Total }\end{array}$ \\
\hline \multicolumn{2}{|l|}{ BamHI/Sall Restriction Digest } \\
\hline $\begin{array}{l}\text { BamHI Enzyme } \\
\text { Sall Enzyme } \\
\text { NE Buffer } 3.1(10 x) \\
\text { DNA Template } \\
\text { dd }_{2} \mathrm{O}\end{array}$ & $\begin{array}{l}0.5 \mu \mathrm{L} \text { (5 units) } \\
0.5 \mu \mathrm{L}(5 \text { units) } \\
2 \mu \mathrm{L}(1 \mathrm{x}) \\
\mathrm{X} \mu \mathrm{L}(\leq 500 \mathrm{ng}) \\
\text { up to } 20 \mu \mathrm{L} \\
\underline{20 \mu \mathrm{L} \text { Total }}\end{array}$ \\
\hline$\underline{\text { T4 DNA Ligation Reaction }}$ & \\
\hline $\begin{array}{l}\text { T4 DNA Ligase Enzyme } \\
\text { Ligation Buffer (10x) } \\
\text { Mixed Digested DNA Templates* } \\
\mathrm{dd}_{2} \mathrm{O}\end{array}$ & $\begin{array}{l}0.5 \mu \mathrm{L} \text { (10 units) } \\
1 \mu \mathrm{L}(1 \mathrm{x}) \\
\mathrm{x} \mu \mathrm{L}(\leq 200 \mathrm{ng}) \\
\text { Up to } 10 \mu \mathrm{L} \\
10 \mu \mathrm{L} \text { Total }\end{array}$ \\
\hline
\end{tabular}

$$
\text { Vector: Template Ligation Ratio }=\frac{x 1}{\mathrm{bp}}: \frac{\mathrm{y} 1}{\mathrm{bp}}
$$

\subsubsection{Electroporation into E. coli Cells}

Plasmids containing the carD flanking region insert were introduced into DH10B E. coli cells (Life Technologies) by electroporation. Electrocompetent E. coli (40 $\mu \mathrm{L})$ were thawed on ice, and then pipetted into pre-chilled $1.5 \mathrm{~mL}$ microcentrifuge tubes on ice. Purified plasmid ( $2 \mu \mathrm{L}, 100$ to $300 \mathrm{ng}$ DNA) was added to each sample with gentle pipetting, and chilled for 30 to 60 seconds on ice. Pre-chilled $0.2 \mathrm{~mm}$ electroporation cuvettes were completely dried and the electroporation mix was pipetted into the cuvettes, gently tapping to remove air bubbles and settle the mixture. A pulse was 
delivered $(25 \mu \mathrm{F}$ capacitance, $2.5 \mathrm{kV} / \mathrm{cm}$, and $200 \Omega$ resistance; Biorad Gene Pulser Xcell), pre-warmed $37^{\circ} \mathrm{C} \mathrm{SOC}(1 \mathrm{~mL})$ was immediately added to the cuvettes cells to recover the cells, and then the mixture was transferred to a fresh microcentrifuge tube. The electroporated cells were incubated for one hour at $37^{\circ} \mathrm{C}$ with shaking to recover cultures. Recovered electroporation mixes were then spread on selective LB agar plates (kanamycin or gentamicin) at varied concentrations and incubated overnight at $37^{\circ} \mathrm{C}$ in a stationary incubator.

\subsubsection{DNA Sequencing and DNA Sequence Analysis}

For sequencing of DNA products, samples were prepared in $20 \mu \mathrm{L}$ aliquots at $\sim 100 \mathrm{ng}$ of total DNA. Samples were shipped to Macrogen (Korea) for sequencing, using Universal M13 primers. Returned sequencing outputs were analysed using Geneious bioinformatics software program $8.1 .7^{[233]}$, multiple sequence alignment tool.

For analysis and comparison of bacterial DNA and protein amino acid sequences, alignments were performed using Clustal Omega, a multiple sequence alignment $\operatorname{program}^{[234]}$.

\subsection{Construction of the pJS01 Plasmid}

To generate the pJS01 plasmid, sequences flanking the carD gene were amplified by PCR. We amplified 0.5 to $0.6 \mathrm{~kb}$ of sequence on either side of the $\operatorname{car} D$ gene, providing enough sequence for homologous recombination to take place. Purified B. henselae gDNA ( 1 to $5 \mu \mathrm{L},<250 \mathrm{ng}$ ) was used as a template for two concurrent PCR reactions (for diagram see Appendix Figure 8.2) using Advantage ${ }^{\circledR}$ Polymerase (PCR components are listed in Table 2.5 and PCR conditions in Table 2.6). The first PCR 
used the prJS01 primer pair to yield the 5' flank product, while the second PCR used the prJS02 forward and reverse primers to yield the 3' flank product (primers are listed in Table 2.1). Both PCR products were predicted to be $0.65 \mathrm{~kb}$. The PCR control reaction with no DNA template was run concurrently.

The resulting PCR products were combined in a 1:1 ratio and used as a template for another PCR reaction, using primers prJS01 forward and prJS02 reverse. Combining both PCR products as a single template enabled internal priming, and then "fusion" of PCR products to occur. The resulting product was predicted to be about $1.3 \mathrm{~kb}$ in size. The PCR fusion product was confirmed by gel electrophoresis, then was subjected to a double digest using BamHI and SalI. The plasmid pEX18gm was simultaneously digested, also with BamHI and SalI, and the digestion products combined and ligated to form the pJS01 plasmid. The ligations were electroporated into DH10B cells, which were selected on LB plates with gentamicin. The resulting plasmid was verified by restriction digest and sequencing (Macrogen), and subsequently used for conjugation of B. henselae. Confirmed pJS01 plasmids were electroporated into S17-1 E. coli donor strain.

\subsection{Construction of the pJS02 Plasmid}

When conjugations with plasmid pJM01 did not result in merodiploid colonies, we explored constructing a mutagenic plasmid with pJM05, which has been used previously to mutagenise Bartonella. Because pJM05 lacks a SalI site in the cloning site, the carD flanking region was only changed to adjust the restriction sites for cloning. The carD flanking region PCR fusion product (described above) was used as a template for a round of PCR using primers prJS01 forward and prJS02 reverse 2; this introduced 
BamHI sites at each end of the PCR fusion product. Because the PCR product was cloned into a single restriction site, the vector was treated with calf intestine alkaline phosphatase to prevent the ends re-joining, following restriction digest. The product was ligated into the BamHI site of pJM05, and the ligation product electroporated into E. coli. Bacteria were selected on kanamycin LB plates, and resulting plasmid was verified by restriction digest. Confirmed pJS02 plasmids were transformed into S17-1 as above.

\subsection{Transformation of Bartonella henselae}

\subsubsection{B. henselae Bi-Parental Conjugation}

Transfer of either the pJS01 or pJS02 plasmid into B. henselae was carried out by bi-parental conjugation of S17-1 and $B$. henselae, essentially as described by Roden et $a l^{[230]}$. S17-1 E. coli containing the plasmid was cultured overnight in fresh selective $\mathrm{LB}$ media $\left(10 \mathrm{~mL}\right.$, kanamycin or gentamicin) in a $50 \mathrm{~mL}$ Falcon tube at $37^{\circ} \mathrm{C}$ with shaking $(250 \mathrm{rpm})$. The following morning, overnight cultures were diluted 1:10 in fresh $\mathrm{LB}$ media (to a volume of $5 \mathrm{~mL}$ ), then returned to the $37^{\circ} \mathrm{C}$ incubator for about three hours. The $\mathrm{OD}_{600}$ was continuously monitored until it reached an $\mathrm{OD}_{600}$ of $1.0\left(\sim 1 \times 10^{9}\right.$ cells $\left./ \mathrm{mL}\right)$. The $\mathrm{S} 17-1$ culture $(1 \mathrm{~mL})$ was washed three times in M199S media by centrifugation for one minute at $10,000 \times \mathrm{g}$. After each centrifugation step, the supernatant was carefully removed with a narrow pipette and pellets were resuspended in fresh M199S (1 mL). After the final centrifugation and re-suspension step, the resuspended pellet was diluted with 1:50 in fresh M199S media (10 $\mu \mathrm{L}$ into $500 \mu \mathrm{L})$. The diluted S17-1 culture containing the plasmid was then pipetted directly (all $510 \mu \mathrm{L}$ ) onto a confluent plate of $B$. henselae. Bacteria were mixed on the plate surface, air-dried 
for five minutes, and then were incubated facing up for five hours in a $5 \% \mathrm{CO}_{2}, 37^{\circ} \mathrm{C}$ stationary incubator, to allow conjugation to occur. Exconjugants were scraped from the plate, resuspended in fresh M199S (500 $\mu \mathrm{L})$, and various dilutions were immediately plated $(150 \mathrm{~mL})$ onto selective chocolate agar plates. The selective plates contained $10 \mu \mathrm{g} / \mathrm{mL}$ gentamicin or $30 \mu \mathrm{g} / \mathrm{mL}$ kanamycin (to select for transconjugants), and $20 \mu \mathrm{g} / \mathrm{mL}$ cefazolin, and $2 \mu \mathrm{g} / \mathrm{mL}$ nalidixic acid, to suppress the growth of E. coli. Selective plates were incubated for 10 to 14 days in a humidified $\mathrm{CO}_{2}, 37^{\circ} \mathrm{C}$ stationary incubator.

\subsubsection{B. henselae Electroporation}

Transformation of mutagenic plasmids into $B$. henselae was carried out as described in the literature $\mathrm{e}^{[235,236]}$, with several adjustments. Electrocompetent $B$. henselae cells $(40 \mu \mathrm{L})$ were thawed on ice, and then pipetted into pre-chilled $1.5 \mathrm{~mL}$ microcentrifuge tubes placed alongside $1 \mathrm{~mm}$ electroporation cuvettes on ice. Purified plasmid ( $2 \mu \mathrm{L}$; 200 to $800 \mathrm{ng}$ of total DNA) was added to each sample with gentle pipetting, and chilled for 30 to 60 seconds on ice. The electroporation mix was pipetted into the cuvettes, gently tapping to remove air bubbles and to settle the mixture. Cuvettes were dried on the outside and inserted into the electroporation machine; a pulse was delivered (25 $\mu \mathrm{F}$ capacitance, $2.5 \mathrm{kV} / \mathrm{cm}$, and $200 \Omega$ resistance). Pre-warmed $37^{\circ} \mathrm{C}$ Schneider's Drosophila Medium containing FCS (1 mL) was added immediately to the cuvettes to recover the cells. The electroporation mixtures were transferred to fresh microcentrifuge tubes and were incubated overnight in a $5 \% \mathrm{CO}_{2}, 35^{\circ} \mathrm{C}$ stationary incubator. Recovered electroporation mixes were spread $(100 \mu \mathrm{L})$ onto selective chocolate agar plates at various concentrations. Selective plates contained $10 \mu \mathrm{g} / \mathrm{mL}$ gentamicin or $30 \mu \mathrm{g} / \mathrm{mL}$ 
kanamycin. Selective plates were incubated for 10 to 14 days in a $5 \% \mathrm{CO}_{2}, 35^{\circ} \mathrm{C}$ incubator.

\subsection{Growth and Stress Response Assays}

\subsubsection{Acclimatised B. henselae Bacterial Suspension Preparation}

Three to four confluent $B$. henselae chocolate agar plates were harvested and resuspended in Schneider's Drosophila Medium containing 10\% FCS (1 mL) in $1.5 \mathrm{~mL}$ microcentrifuge tubes (one tube per plate). Resuspended $B$. henselae cultures were washed three times in Schneider's Medium via centrifugation for five minutes at 3,000 x $g$. After each centrifugation step, the supernatant was carefully removed with a narrow pipette and pellets were resuspended in fresh Schneider's Medium containing FCS $(1 \mathrm{~mL})$. After the final centrifugation and re-suspension step, the washed suspensions were combined in a $50 \mathrm{~mL}$ Falcon tube then diluted into pre-warmed $37^{\circ} \mathrm{C}$ Schneider's Medium containing FCS to an $\mathrm{OD}_{600}$ equal to 1.0. The diluted $B$. henselae suspension was then incubated for two hours in a humidified $5 \% \mathrm{CO}_{2}, 37^{\circ} \mathrm{C}$ stationary incubator to acclimate the bacteria to the liquid culture condition. The acclimatised $B$. henselae suspension was further diluted either 1:10 for growth assays or 3:10 for stress assays, and divided into replicates in $50 \mathrm{~mL}$ Falcon tubes using pre-warmed $37^{\circ} \mathrm{C}$ Schneider's Medium containing FCS. The result was B. henselae bacterial suspensions in Schneider's Medium containing FCS at an $\mathrm{OD}_{600}$ of 0.1 or 0.3 . Each growth assay replicate was carried out in a volume of $30 \mathrm{~mL}$, while each stress assay replicate was carried out in a volume of $10 \mathrm{~mL}$. 


\subsubsection{Baseline Growth Control}

Three acclimatised B. henselae bacterial suspensions in Schneider's Drosophila Medium containing FCS (at an $\mathrm{OD}_{600}$ of 0.1 , of a volume of $30 \mathrm{~mL}$ ) were prepared as above, and incubated for 14 days in a humidified $\mathrm{CO}_{2}, 37^{\circ} \mathrm{C}$ incubator; the $\mathrm{OD}_{600}$ was taken for 14 days to generate a growth curve using GraphPad Prism Version 6.0 graphing software.

\subsubsection{Low Temperature Growth Assay}

Three acclimatised B. henselae bacterial suspensions in Schneider's Drosophila Medium containing FCS (at an $\mathrm{OD}_{600}$ of 0.1 , of a volume of $30 \mathrm{~mL}$ ) were prepared as above, with the following adjustments. Each suspension was diluted with pre-warmed $28^{\circ} \mathrm{C}$ Schneider's Medium containing FCS, and suspensions were incubated in a $\mathrm{CO}_{2}$ GENbox (BioMérieux) inside a humidified $28^{\circ} \mathrm{C}$ stationary incubator; the $\mathrm{OD}_{600}$ was taken once daily for 14 days to generate a growth curve as above.

\subsubsection{Haemin Stress Assay}

Three acclimatised B. henselae bacterial suspensions in Schneider's Drosophila Medium containing FCS (at an $\mathrm{OD}_{600}$ equal to 0.3 , of a volume of $10 \mathrm{~mL}$ ) were prepared as above and treated with haemin. The first suspension was a non-supplemented control, with the remaining two $B$. henselae suspensions supplemented with haemin (Sigma) at $0.1 \mathrm{mM}$ and $0.5 \mathrm{mM}$ respectively. Suspensions were incubated for 24 hours in a humidified $5 \% \mathrm{CO}_{2}, 37^{\circ} \mathrm{C}$ stationary incubator, during which samples were taken regularly (twice from each suspension). Each sample was serially diluted in Schneider's Medium containing FCS; dilutions were streaked $(100 \mu \mathrm{L}$ per dilution) onto plain chocolate agar plates, and then incubated for 14 days in $\mathrm{CO}_{2}, 37^{\circ} \mathrm{C}$ incubator. Colonies on each plate were counted by hand or using colony counting software (OpenCFU ${ }^{[237]}$ ) 
to determine rate of survival in each haemin condition, which was graphed using GraphPad Prism Version 6.0.

\subsubsection{Ciprofloxacin Assay}

Acclimatised B. henselae bacterial suspensions in Schneider's Drosophila Medium containing FCS (at an $\mathrm{OD}_{600}$ equal to 0.3 , of a volume of $10 \mathrm{~mL}$ ) were prepared as above and treated with ciprofloxacin (Sigma). Alongside an untreated control, bacterial suspensions were supplemented with ciprofloxacin at $10 \mu \mathrm{g} / \mathrm{mL}$ or $25 \mu \mathrm{g} / \mathrm{mL}$. Suspensions were incubated for 10 hours in a $5 \% \mathrm{CO}_{2}, 37^{\circ} \mathrm{C}$ incubator, during which samples were taken regularly; each sample was prepared by serial dilution as before, plated onto plain chocolate agar plates, and then incubated for 14 days in a $\mathrm{CO}_{2}, 37^{\circ} \mathrm{C}$ incubator. Colonies were counted and the rate of survival determined as for haemin above.

\subsubsection{Hydrogen Peroxide $\left(\mathrm{H}_{2} \mathrm{O}_{2}\right)$ Assay}

Acclimatised B. henselae bacterial suspensions in Schneider's Drosophila Medium containing FCS (at an $\mathrm{OD}_{600}$ equal to 0.3 , of a volume of $10 \mathrm{~mL}$ ) were prepared as above and treated with $\mathrm{H}_{2} \mathrm{O}_{2}$. Alongside the untreated control, the remaining bacterial suspensions were supplemented with $\mathrm{H}_{2} \mathrm{O}_{2}$ at $0.1 \mathrm{mM}$ or $1 \mathrm{mM}$ and then incubated for one and a half hours in a $5 \% \mathrm{CO}_{2}, 37^{\circ} \mathrm{C}$ incubator. As for previous assays, samples were taken regularly throughout the incubation, serially diluted onto chocolate agar and incubated for 14 days in a $\mathrm{CO}_{2}, 37^{\circ} \mathrm{C}$ incubator. Resulting colonies were counted and the rate of survival was determined as for haemin-treated colonies as above. 


\subsubsection{Starvation Assay}

An undivided, acclimatised B. henselae suspension in Schneider's Drosophila Medium containing FCS (at an $\mathrm{OD}_{600}$ equal to 1) was washed a further three times in 1x PBS via centrifugation to remove all nutrients. B. henselae suspended in pre-warmed $37^{\circ} \mathrm{C}$ 1x PBS was divided into three aliquots and diluted to reach an $\mathrm{OD}_{600}$ of 0.3 , with one suspension diluted in Schneider's Medium containing FCS to act as a control, and two suspensions diluted in 1x PBS; suspensions were incubated for 10 hours in a $5 \% \mathrm{CO}_{2}$, $37^{\circ} \mathrm{C}$ incubator. Samples were regularly taken from each suspension, serially diluted and then plated onto chocolate agar plates for incubation, 14 days at $\mathrm{CO}_{2}, 37^{\circ} \mathrm{C}$. Colonies were counted and the rate of survival was determined as for haemin assays above.

\subsection{1. qPCR Analysis of carD Stress Induced Expression}

\subsubsection{Selection of $B$. henselae Stress Conditions}

The results of the $B$. henselae stress condition assays were analysed, and for each stress condition, the concentration of stressor and length of stress exposure were chosen to correspond with $B$. henselae survival of 80 to $90 \%$. The only exception was for the $37^{\circ} \mathrm{C}$ and low temperature growth assays, where the bacteria continued to replicate. All the stress conditions are listed in Table 2.8 below. 
Table 2.8. - B. henselae Stressors and Stressor Conditions

\begin{tabular}{lll}
\hline Stress Condition & Supplement Concentration & Time of Stress Exposure \\
\hline \hline Baseline Growth Control $\left(37^{\circ} \mathrm{C}\right)$ & N/A & Two Hours \\
Low Temperature $\left(28^{\circ} \mathrm{C}\right)$ & N/A & Two Hours \\
Haemin & $0.1 \mathrm{mM}$ & One Hour \\
Ciprofloxacin & $10 \mathrm{ng} / \mu \mathrm{L}$ & One Hour \\
Hydrogen Peroxide $\left(\mathrm{H}_{2} \mathrm{O}_{2}\right)$ & $0.1 \mathrm{mM}$ & 10 Minutes \\
Starvation & PBS & 30 Minutes \\
\hline
\end{tabular}

\subsubsection{Collection of $B$. henselae Cultures under Treatment Conditions}

Acclimatised B. henselae in Schneider's Drosophila Medium containing FCS (at an $\mathrm{OD}_{600}$ of 1.0$)$ were prepared from six to seven confluent $B$. henselae plates. The suspension was diluted by 3:10 and divided into six volumes in $50 \mathrm{~mL}$ Falcon tubes using pre-warmed Schneider's Medium containing FCS (five at $37^{\circ} \mathrm{C}$ and one at $28^{\circ} \mathrm{C}$, each at a volume of 10 to $15 \mathrm{~mL}$ ). The result was six suspensions of $B$. henselae in Schneider's Medium containing FCS at an $\mathrm{OD}_{600}$ equal to 0.3. Each B. henselae culture was supplemented with their respective stressor as indicated in Table $\mathbf{2 . 8}$ and incubated at $5 \% \mathrm{CO}_{2}, 37^{\circ} \mathrm{C}$ for the indicated time (or for low temperature, at $28^{\circ} \mathrm{C}$ in a $\mathrm{CO}_{2}$ GENbox). In the case of the starvation condition, the culture was washed in 1x PBS via centrifugation as per above and then incubated at $5 \% \mathrm{CO}_{2}, 37^{\circ} \mathrm{C}$.

After the cultures were exposed to the stressor for the designated time, a volume of each culture containing approximately $1 \times 10^{8}$ bacteria was combined with a RNA stop solution ( $100 \mu \mathrm{L}$ of $5 \%$ phenol in absolute ethanol) in $1.5 \mu \mathrm{L}$ microcentrifuge tubes to prevent additional transcription of RNA. Cultures were mixed by inversion and immediately centrifuged at maximum speed for two to three minutes to pellet bacteria. The supernatant was removed by pipetting and the pellets were immediately frozen at $-80^{\circ} \mathrm{C}$ freezer overnight. 


\subsubsection{B. henselae RNA Extraction using TRIzol and RNeasy}

All RNA work was performed using RNase-free reagents in a biosafety cabinet. Pelleted B. henselae colonies from each stress condition were removed from the $-80^{\circ} \mathrm{C}$ freezer and briefly thawed at room temperature. Each pellet was resuspended in lysozyme (50 $\mu \mathrm{L}$ of $0.4 \mathrm{mg} / \mathrm{mL}$ lysozyme in TE; Sigma) with gentle pipetting and incubated for five minutes at room temperature to weaken the cell wall. TRIzol reagent $(0.95 \mathrm{~mL}$; Life Technologies) was added to each microcentrifuge tube, vortexed for 30 to 60 seconds, and then incubated for five minutes at room temperature. Chloroform $(200 \mu \mathrm{L})$ was added to each microcentrifuge tube; tubes were sealed and shaken by hand for 15 seconds, and then incubated for two to three minutes at room temperature. Phases were separated by centrifugation for 15 minutes at $12,000 \mathrm{x}$ g. Taking care not to disturb the middle or lower phases, the colourless upper phase containing RNA was pipetted into fresh $50 \mathrm{~mL}$ falcon tubes, with one Falcon tube per stress condition; up to four bacterial pellets for each sample were combined.

To prepare the RNA suspensions for RNeasy® (Qiagen) columns, absolute ethanol (of a volume equivalent to 0.5 of the volume of RNA) was slowly added to each $50 \mathrm{~mL}$ Falcon tube via a pipette whilst vortexing at the lowest setting to mix. The RNA-ethanol suspension was loaded onto RNeasy ${ }^{\circledR}$ columns in sequential $700 \mu \mathrm{L}$ aliquots, and centrifuged at maximum speed for 30 seconds after each aliquot addition, discarding the flow-through after each spin. Once all the RNA was loaded onto the columns, Buffer RW1 $(0.35 \mathrm{~mL})$ was added to the column and centrifuged at maximum speed for 30 seconds, discarding the flow-through. An on-column DNA digestion using DNase I (Qiagen) was then performed. For each column, $10 \mu \mathrm{l}$ of DNase I stock solution was combined with $70 \mu \mathrm{l}$ Buffer RDD, and the total $(80 \mu \mathrm{l})$ was added to each column 
and incubated for 30 minutes at room temperature. Buffer RW1 $(0.35 \mathrm{~mL})$ was added to each column, followed by centrifugation for 30 seconds at maximum speed, discarding flow through. Buffer RPE $(500 \mu \mathrm{L})$ was added to each column twice; the first spin was for 30 seconds at maximum speed, while the second spin was for two minutes. The empty columns were spun for a further minute to dry the columns. To elute the RNA, RNA-free $\mathrm{H}_{2} \mathrm{O}(50 \mu \mathrm{L})$ was added to the centre of each column, incubated for two minutes at room temperature, and then centrifuged for one minute at maximum speed. A small portion of RNA ( 3 to $5 \mu \mathrm{l}$ ) was placed in a new tube for testing, and the remainder was stored at $-80^{\circ} \mathrm{C}$. RNeasy® purified RNA was analysed for concentration and quality via NanoDrop.

\subsubsection{Synthesis of cDNA Using Reverse Transcriptase}

Synthesis of cDNA products was performed using GoScript ${ }^{\mathrm{TM}}$ Reverse Transcription System (Promega). The RNA and a random hexamer primer were combined (reaction components and volumes are listed in Table 2.9) in 0.2 mL PCR tubes and incubated for five minutes at $70^{\circ} \mathrm{C}$ to denature any RNA secondary structures, then chilled on ice for five minutes. Reverse transcription reaction mixtures for cDNA were prepared and added to the primer/RNA mix. For the reverse transcription reaction, $15 \mu \mathrm{L}$ of reverse transcription reaction mixture was added to each $5 \mu \mathrm{L}$ of first strand synthesis mixture, then the combined reaction mixture was incubated for five minutes at $25^{\circ} \mathrm{C}$ (annealing), for one hour at $42^{\circ} \mathrm{C}$ (extension), and for 15 minutes at $70^{\circ} \mathrm{C}$ (inactivation). Extended cDNA fragments were stored at $-20^{\circ} \mathrm{C}$. Negative controls, which included reverse transcription reactions with no template and, for each stress condition, a sample with no reverse transcriptase added, were run concurrently. 


\begin{tabular}{|c|c|}
\hline Reaction Components & Quantity \\
\hline \multicolumn{2}{|l|}{$\underline{\text { RNA and Primer Mixture }}$} \\
\hline $\begin{array}{l}\text { RNA Template } \\
\text { Random hexamer Primers }(10 \mu \mathrm{g}) \\
\text { Nuclease-Free } \mathrm{H}_{2} \mathrm{O}\end{array}$ & $\begin{array}{l}x \mu \mathrm{L}(100 \text { to } 300 \text { total } \mathrm{ng}) \\
1 \mu \mathrm{L}(0.5 \mu \mathrm{g}) \\
\text { up to } 5 \mu \mathrm{L} \\
\underline{5 \mathrm{~L} \text { Total }}\end{array}$ \\
\hline \multicolumn{2}{|l|}{ Reverse Transcription Reaction Mixture } \\
\hline $\begin{array}{l}\text { Nuclease-Free } \mathrm{H}_{2} \mathrm{O} \\
\mathrm{GoScript}^{\mathrm{TM}} \text { Reaction Buffer }(5 \mathrm{x}) \\
\mathrm{MgCl}_{2}(25 \mathrm{mM}) \\
\text { Nucleotide Mix }(10 \mu \mathrm{g}) \\
\text { RNasin }^{\circledast} \text { Ribonuclease Inhibitor } \\
\text { GoScript }^{\mathrm{TM}} \text { Reverse Transcriptase (20x) }\end{array}$ & $\begin{array}{l}7 \mu \mathrm{L} \\
4 \mu \mathrm{L}(1 \mathrm{x}) \\
1 \mu \mathrm{L}(1.25 \mathrm{mM}) \\
1 \mu \mathrm{L}(0.5 \mathrm{mM}) \\
1 \mu \mathrm{L}(20 \text { units }) \\
1 \mu \mathrm{L}(1 \mathrm{x})\end{array}$ \\
\hline & $15 \mu \mathrm{L}$ Total (per reaction) \\
\hline
\end{tabular}

\subsubsection{Quality Control of cDNA Products}

To confirm successful reverse transcription and that the RNA samples lacked genomic DNA contamination, a portion of each cDNA product $(2 \mu \mathrm{L})$ was used as a template for two consecutive PCR reactions with Phusion ${ }^{\circledR}$ High-Fidelity PCR Polymerase Mastermix (New England Biolabs; PCR components and conditions are listed in Table 2.5 and Table 2.6. The housekeeping ribosomal gene, $r p s L$, was used as an internal control. All samples were amplified separately by PCR using the carD prJS03 primer pair or the rpsL prJS04 primer pair (all primers are listed in Table 2.1). PCR control reactions with no reverse transcriptase, no template, or with $B$. henselae gDNA, were run concurrently. PCR products were run on agarose electrophoresis gels alongside Hyperladder I and analysed under UV light. 


\subsection{6. $\quad$ qPCR Quantification of $B$. henselae carD Expression}

For qPCR reactions, KAPA SYBR® FAST Kit (KAPA Biosystems) was used in a CFX96 ${ }^{\mathrm{TM}}$ Real-Time PCR Detection System machine (Biorad). For each $B$. henselae stress condition, two qPCR reactions were performed in duplicate. The first used carD gene-specific pJS03 primers to determine the level of $\operatorname{carD}$ expression, and the second used rpsL housekeeping gene pJS04 primers as a baseline control. qPCR control reactions with no reverse transcriptase (for each stress condition), and a no template control were run concurrently with both $\operatorname{carD}$ and $\operatorname{rps} L$ primers.

Reaction mixtures for qPCR were prepared as below, first thawing components on ice, and then combining in the order listed in Table 2.10, in $0.2 \mathrm{~mL}$ PCR tubes with optically clear caps. Samples were run using the qPCR cycling conditions listed in Table 2.11, and the results analysed using CFX Manager ${ }^{\mathrm{TM}} 3.0$ (Biorad) and using GraphPad Prism Version 6.0.

Table 2.10. - Standard qPCR Components and Quantities

\begin{tabular}{ll}
\hline Reaction Components & Quantity \\
\hline \hline qPCR Reaction Mixtures & \\
Nuclease-Free $\mathrm{H}_{2} \mathrm{O}$ & $4 \mu \mathrm{L}$ \\
KAPA SYBR FAST Mastermix $(2 \mathrm{x})$ & $10 \mu \mathrm{L}(1 \mathrm{x})$ \\
cDNA Template $(5 \mathrm{ng} / \mu \mathrm{L})$ & $2 \mu \mathrm{L}(10 \mathrm{ng}$ Total) \\
Forward Primer $(2 \mu \mathrm{M})$ & $2 \mu \mathrm{L}(200 \mathrm{nM})$ \\
Reverse Primer $(2 \mu \mathrm{M})$ & $2 \mu \mathrm{L}(200 \mathrm{nM})$ \\
& $\underline{20 \mu \mathrm{L} \text { Total }}$ \\
\hline
\end{tabular}

Table 2.11. - Standard qPCR Conditions

\begin{tabular}{cccc}
\hline qPCR Conditions & Temperature & Hold Time & Cycle Number \\
\hline \hline qPCR Conditions & & & \\
Pre-heat & $95^{\circ} \mathrm{C}$ & 3 Minutes & \\
Denaturation & $95^{\circ} \mathrm{C}$ & 3 Seconds & 40 cycles \\
Annealing/Extension & $58^{\circ} \mathrm{C}$ & 20 Seconds & \\
Dissociation Curve & 60 to $95^{\circ} \mathrm{C}$ in $0.5^{\circ} \mathrm{C}$ & 0.05 Seconds per & \\
& increments & increment & \\
& &
\end{tabular}




\section{Chapter 3. Construction of a Bartonella henselae carD Markerless Deletion Strain}

\subsection{Introduction}

The aim of this chapter was to generate a $B$. henselae $\triangle$ carD mutant strain to compare the growth rate to that of the B. henselae wild type strain under stress conditions.

CarD plays an important role in Mycobacterium tuberculosis, Myxococcus xanthus, and Thermus thermophilus bacteria, in global regulation of mRNA and in DNA repair; in these bacteria $c a r D$ is up-regulated in response to DNA strand breaks caused by endonucleases and genotoxic stresses ${ }^{[212]}$. The CarD amino acid sequence homology between $B$. henselae, $M$. tuberculosis, M. xanthus, and T. thermophilus is significant; a NCBI Blast query showed that B. henselae CarD (NCBI: WP_011181290.1) shared $30.0 \%, 35.0 \%$, and $36.0 \%$ sequence similarity with $M$. tuberculosis CarD (NCBI: WP_057133327.1), M. xanthus CdnL (carD equivalent; NCBI: 2LWJ_A) and T. thermophilus carD (NCBI: WP_011172978.1) respectively (for amino acid sequence alignments see Appendix Figure 8.1). Due to the level of sequence similarity between well studied bacterial species and Bartonella $\mathrm{CarD}$, we hypothesised that CarD carries out similar functions in Bartonella species.

A common method of analysing specific gene function is by generating a mutant bacterial strain through targeted gene deletion. Though this method is not viable in Mycobacterium and Myxococcus as carD is required for growth, this does not appear to hold true for carD in Bartonella birtlesii ${ }^{[176]}$, and potentially other Bartonella species. As such we aimed to generate a $B$. henselae $\Delta c a r D$ strain. 


\subsection{Results}

To generate a $B$. henselae $\Delta c a r D$ bacterial strain, we opted to use a targeted approach similar to those previously published for Bartonella ${ }^{[228,235,236]}$. Briefly, genomic sequences flanking the $B$. henselae carD gene were amplified and then fused together by PCR. The carD PCR fusion product was inserted into a suicide plasmid, lacking an origin of replication that functions in Bartonella, and transferred into B. henselae through either bi-parental conjugation or electroporation. The carD flanking sequences were long enough to enable homologous recombination between plasmid and B. henselae DNA, the result of which was the integration of the plasmid into the chromosome next to $c a r D$, resulting in a merodiploid gene (for a diagram of recombination, see Figure 3.1). B. henselae cells with the integrated plasmid were selected for by antibiotic selection. The integrated plasmid encodes a $s a c B$ gene, which enables negative selection and loss of the integrated plasmid, following growth on selective media containing sucrose ${ }^{[228,238]}$. The loss of the plasmid, by a second homologous recombination event, may either result in restoration of the wild type $\operatorname{car} D$ gene, or complete loss of the gene, resulting in a null mutant. The goal of this project was to assess the susceptibility of the resulting $\Delta \operatorname{carD} B$. henselae mutant strain to environmental stresses, including low temperature $\left(28^{\circ} \mathrm{C}\right)$, haemin exposure, genotoxic stresses (ciprofloxacin and hydrogen peroxide), and nutrient starvation. 
Chromosomal B.henselae (Bh) - carD

$5^{\prime}$

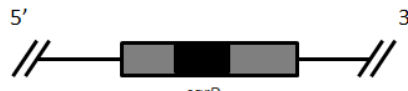

Plasmid - carD

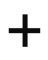

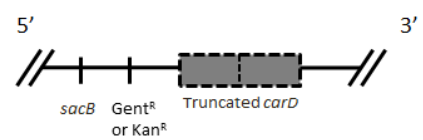

b.

Recombination of Plasmid into B. henselae Chromosome (Merodiploid Positive Selection)

Bh and Plasmid carD - carD Recombination

$5^{\prime}$

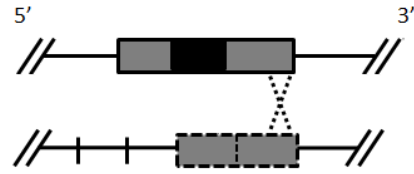

Chromosomal Bh- Merodiploid carD

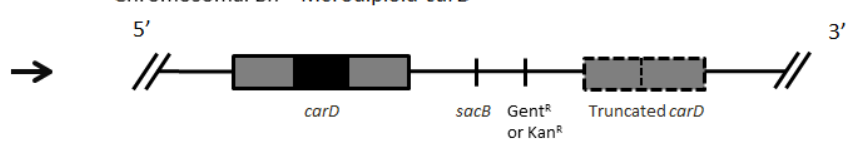

c.

Chromosomal B. henselae carD-carD Internal Recombination (Merodiploid Negative Selection)
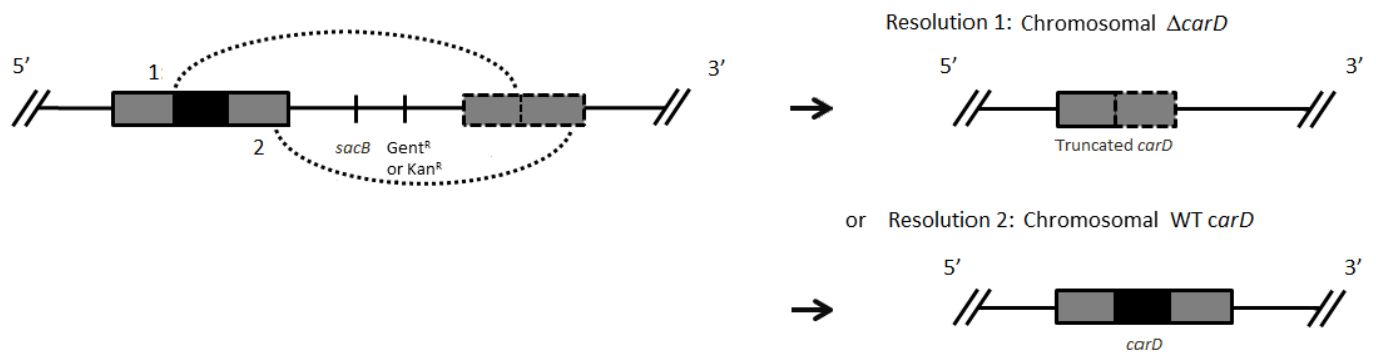

Figure 3.1. - Mutagenesis and selection of the carD mutant (a.) Successful transfer of the plasmid through electroporation or conjugation leads to the presence of $\operatorname{carD}$ in both the suicide plasmid and in B. henselae chromosomal DNA. (b.) Through merodiploid positive selection with antibiotics, only $B$. henselae with an integrated plasmid grows. Chromosomal carD and truncated $\operatorname{car} D$ have a crossover and recombination event that leads to chromosomal insertion of the plasmid alongside carD, as the plasmid cannot persist alone in the cell. (c.) Through merodiploid negative selection, only $B$. henselae that have lost the $\operatorname{sac} B$ gene can grow. The gene was lost via one of two possible carD-carD recombination events (see $c$, dotted lines, area covered by arc is lost) resulting in the loss of the plasmid (and $s a c B$ ). Both resolutions lack both $s a c B$ and genes conferring resistance to gentamicin or kanamycin, although resolution 1 has resulted in a $\triangle c a r D$ mutant while resolution 2 results in restoration of a wild type carD. 


\subsubsection{Generation of the Mutagenic Plasmid pJS01}

In selecting an appropriate candidate for the starting plasmid to construct our $B$. henselae mutagenic plasmid, several criteria needed to be met. For conjugation to be successful, the mutagenic plasmid needed to possess an origin of transfer (oriT), and a set of mobility genes ${ }^{[239]}$. The resulting plasmid must also have the requisite genes for selection of bacterial colonies, including an antibacterial resistance gene that functions in Bartonella and a sucrose-selective $s a c B$ gene. The plasmid pEX18gm ${ }^{[226]}$ met all the above criteria, and was selected to be the base of our mutagenic pJS01 plasmid.

To generate the pJS01 plasmid, several successive stages of PCR were utilised. For the first stage, flanking regions of the $B$. henselae carD gene were amplified by PCR. These flanking regions were of sufficient size to allow homologous recombination within these regions to occur. The flanking regions were fused via a subsequent PCR step, resulting in an in-frame deletion of the majority of the carD gene as shown below (for a diagram of the generation of $c a r D$ fusion products, see Appendix Figure 8.2). The fusion product resulted in loss of 347 of 585 base pairs of the carD gene, leaving 258 base pairs of the carD gene after accounting for the addition of primer overhangs used to fuse the 3' and 5' flanking products (for the $c a r D$ gene sequence see Appendix Figure 8.3). No frame shift in amino acid sequence was observed, with 86 amino acids predicted to remain in the mutant. The carD PCR fusion product was inserted into the pEX18gm plasmid by restriction digest and ligation, to yield the pJS01 plasmid. The resulting plasmid was verified by restriction digest, as shown in Figure 8.2, and sequencing (for pJS01 sequence alignments see Appendix Figure 8.4). The plasmid was then transformed into S17-1, and the E. coli strain was then unsuccessfully used for biparental conjugations. 
a)

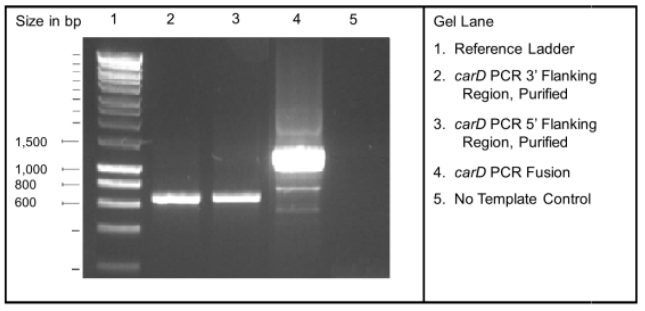

c)

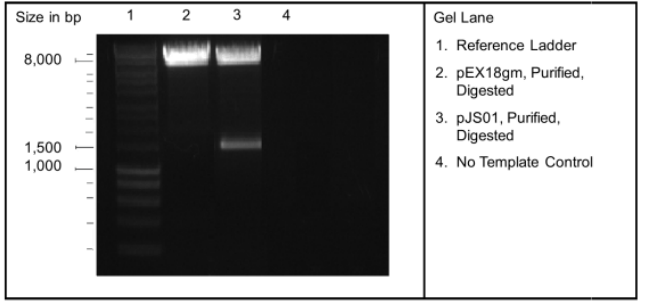

b)

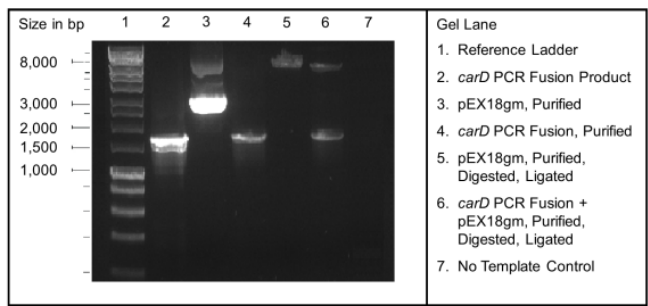

d)

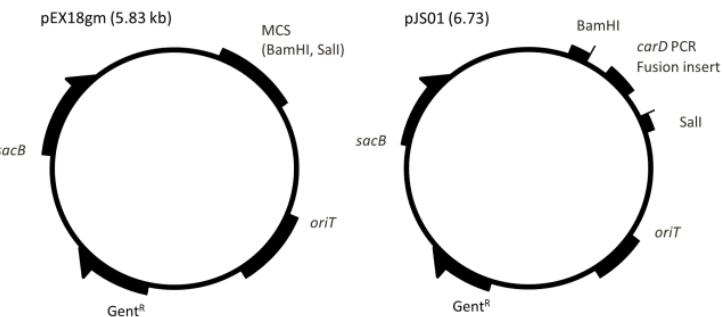

Figure 3.2. - pEX18gm and pJS01 Agarose Electrophoresis Gels and Bacterial Plasmid Maps a) Products from the first two rounds of PCR. The $5^{\prime}$ and $3^{\prime}$ flanking regions were amplified, purified and used as the template for the fusion PCR. Both PCR products were estimated to be $\sim 650$ base pairs, while the carD fusion product was estimated to be $\sim 1,300$ base pairs in size, as seen above. Products of non-specific DNA amplification were removed from the PCR fusion sample by gel purification. b) The PCR fusion and the pEX18gm products were purified, digested and ligated together. c) Ligated PCR fusion and pEX18gm products were transformed into an $E$. coli cloning strain, then purified and digested, confirming that pJS01 had the correct insert. d) Plasmid maps of pEX18gm and pJS01. Sites of interest in pEX18gm include: oriT (origin of transfer), $s a c B$ (sucrose sensitivity), Gent ${ }^{R}$ (gentamicin resistance), and a multiple cloning site (MCS) containing both BamHI and Sall restriction sites. The pJS01 plasmid contains the same sites of pEX18gm, with the addition of the carD gene sequence insert (carD PCR fusion insert) at the MCS, to enable homologous recombination and allelic replacement of the carD gene.

\subsubsection{Generation of the Mutagenic Plasmid pJSO2}

As transformation with pJS01 into $B$. henselae did not yield colonies, we chose to explore other options. Though pEX18gm met all the criteria of our $B$. henselae transformation and mutation experiments, this plasmid had not previously been used for B. henselae mutagenesis; therefore, a second mutagenic plasmid was constructed, starting with a plasmid, pJM05, that was derived from the Bartonella mutagenesis plasmid pRS14, and that had been previously used to successfully mutagenise Bartonella ${ }^{[227,228,240]}$. A sample of pJM05 plasmid DNA was kindly provided by Dr Jane Koehler (UCSF, California), and used to construct our mutagenic plasmid, pJS02. 
The methodologies of constructing the pJS01 and pJS02 plasmids were similar, but due to the differences in restriction sites between pEX18gm and pJM05, minor adjustments were required. Unlike pEX18gm, pJM05 lacks a SalI restriction enzyme site within its multiple cloning site; the $c a r D$ PCR fusion product used to generate pJS01 could not be used to generate pJS02. The carD PCR fusion product was remade essentially as before, but with different restriction enzyme sites.

a)

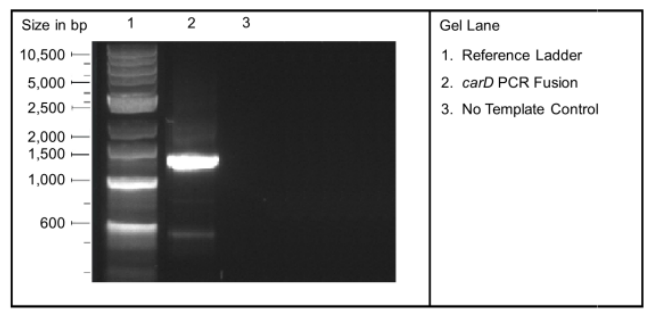

c)

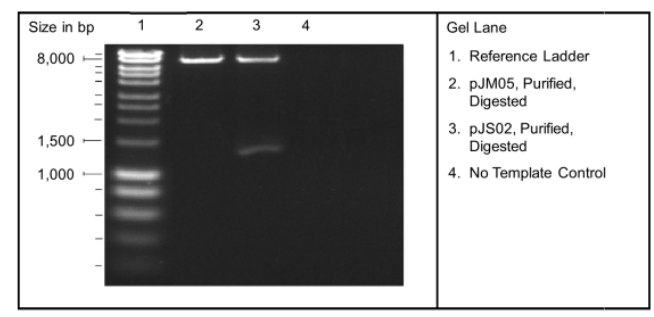

b)

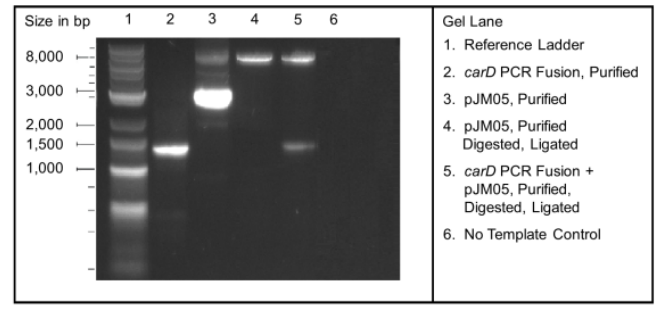

d)

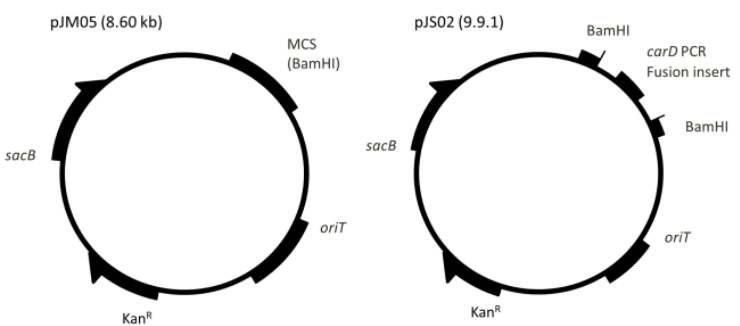

Figure 3.3. - pJM05 and pJS02 Agarose Electrophoresis Gels and Bacterial Plasmid Maps a) The carD fusion product used to generate pJS01 was used as a template to form the new carD PCR fusion product, both of which were estimated to be $\sim 1,300$ base pairs in size, as seen above. b) The purified PCR fusion product and pJM05 were digested with BamHI and ligated together. As just the BamHI enzyme was used, only the new carD PCR fusion product is integrated into pJM05. c) The resulting pJS02 plasmid was purified and analysed by restriction digest. d) Plasmid maps of pJM05 and pJS02. Sites of interest in pJM05 include: oriT, $s a c B, \mathrm{Kan}^{\mathrm{R}}$ (kanamycin resistance), and a multiple cloning site (MCS) containing a BamHI restriction site. The pJSO2 plasmid contains the same sites as pJM05, with the addition of the carD gene sequence insert (carD PCR fusion insert) at the MCS. 


\subsubsection{Transformation of $B$. henselae}

For transformation of $B$. henselae, we turned to the Bartonella literature for protocols. The two techniques commonly used in Bartonella mutagenesis protocols to transform a plasmid into the bacteria are bacterial conjugation and electroporation.

Bi-parental conjugation is a method of horizontal gene transfer via a pilus extended from the donor to the recipient cell, wherein the plasmid is linearized and transferred as single-stranded DNA into the recipient bacteria. For a successful conjugation, the RP4 conjugal transfer genes must be expressed in the donor strain. For a donor strain, we used S17-1 $\lambda$ pir E. coli, which has these genes integrated onto the chromosome ${ }^{[241,242]}$. In preparation for the transformation, both pJS01 and pJS02 were seperately transferred into the S17-1 E. coli donor strain via electroporation. However, we observed that the resulting S17-1 strain with the plasmid had a reduced growth rate. We normally observed that S17-1 strains, carrying other plasmids, will reach an optical density of 1.0 after about one and a half hours, following the 1:10 dilution of an overnight culture. However, S17-1 with pJS01 or pJS02 would typically reach a lower $\mathrm{OD}_{600}$ after overnight growth, and typically required 4 hours or more to reach an $\mathrm{OD}_{600}$ of 1 when 1:10 dilutions were made the following morning. The significance of this slow growth rate is not fully understood, although it may be due to latent transcription of the carD gene.

In the Bartonella literature, bi-parental mating has been reported ${ }^{[230,243]}$. Briefly, cultures of S17-1 containing the pJS01 plasmid were grown, washed to remove antibiotics, and added to confluent $B$. henselae plates; $E$. coli and $B$. henselae cells were incubated together to allow for conjugation, and then the exconjugants were harvested and replated on fresh selective chocolate agar plates to select for transconjugant merodiploid 
cells. After incubation, no resulting merodiploid colonies were found (attempted variations to the stated protocol are listed below). When repeated conjugations with plasmid pJS01 did not result in colonies, we opted to try conjugations using pJS02, a mutagenic plasmid constructed from pJM05, which has been used successfully to make B. quintana and B. henselae mutants numerous times ${ }^{[228,240]}$. Despite repeated attempts, no transconjugant merodiploid colonies appeared after multiple iterations using either mutagenic plasmid, or following introduction of any of the variations listed.

When the pJS01 and pJS02 plasmids were constructed and transformed into S17-1 for biparental conjugation, we observed that the resulting S17-1 strains containing the plasmids had a slow growth rate; though pJS01 growth was never compared against the base pEX18gm plasmid, growth of S17-1 carrying pJS02 was found to be significantly slower when compared to S17-1 carrying pJM05 or other plasmids (data not shown). This indicates that our pJS01 and JS02 plasmids may have detrimental effects on E. coli growth, which could interfere with transformation efficiency. It has been observed in other labs that cultures of S17-1 containing a mutagenic plasmid can exhibit a slowed growth rate, yet still be viable for conjugation (Dr Jane Koehler, personal communication). 
Table 3.1. - Bi-Parental Conjugation Experimental Variations Each experimental step that was varied above was performed in conjunction with the regular protocol steps, and performed in duplicate alongside the regular conjugation protocol. When all options were exhausted with the pJS01 plasmid, the pJSO2 plasmid was created and used in all future conjugation experiments; some, but not all of the above variations were tested using the pJSO2 plasmid.

\begin{tabular}{|c|c|c|}
\hline Experiment Step & Regular Protocol & Variation \\
\hline S17-1 Culture Preparation & $\begin{array}{l}\text { S17-1 cultures were washed three times } \\
\text { with M199S at } 10,000 \times g\end{array}$ & $\begin{array}{l}\text { Washed as before, with 1x PBS instead of } \\
\text { M199S }\end{array}$ \\
\hline $\begin{array}{l}\text { Confluent B. henselae Culture } \\
\text { Preparation }\end{array}$ & $\begin{array}{l}\text { B. henselae were unwashed and left on } \\
\text { the confluent plate before addition of } \\
\text { S17-1 }\end{array}$ & $\begin{array}{l}\text { Bacteria were harvested and washed once in } \\
\text { M199S Medium at } 10,000 \times \mathrm{g} \text {, then re- } \\
\text { plated onto fresh chocolate plates alongside } \\
\text { S17-1 }\end{array}$ \\
\hline S17-1 Conjugation Concentration & $\begin{array}{l}\text { S17-1 culture (in M199S at an } O D_{600} \text { of } \\
\text { 1) was diluted by 1:50 before } \\
\text { conjugation }\end{array}$ & $\begin{array}{l}\text { Cultures are diluted by } 1: 30 \text { and } 1: 70 \\
\text { dilutions }\end{array}$ \\
\hline Conjugation Incubation Length & $\begin{array}{l}\text { B. henselae and } \mathrm{S} 17-1 \text { conjugation } \\
\text { mixtures were incubated for five hours } \\
\text { in a } 5 \% \mathrm{CO}_{2}, 37^{\circ} \mathrm{C} \text { incubator }\end{array}$ & $\begin{array}{l}\text { Conjugation incubations were extended to } \\
\text { eight hours or overnight }\end{array}$ \\
\hline Exconjugant Incubation Length & $\begin{array}{l}\text { Harvested cultures were plated and } \\
\text { incubated on fresh selective media for } \\
10 \text { to } 14 \text { days }\end{array}$ & $\begin{array}{l}\text { Cultures were prepared as before, } \\
\text { incubation time was increased, } 14 \text { to } 20 \text { days }\end{array}$ \\
\hline $\begin{array}{l}\text { Exconjugant Incubation } \mathrm{CO}_{2} \\
\text { Conditions }\end{array}$ & $\begin{array}{l}\text { Culture were incubated in a } 5 \% \mathrm{CO}_{2} \\
37^{\circ} \mathrm{C} \text { Incubator }\end{array}$ & $\begin{array}{l}\text { Selective plates were sealed inside either a } \\
5 \% \mathrm{CO}_{2} \mathrm{GENbox} \text {, a } 5 \% \mathrm{CO}_{2} \mathrm{GENbag} \text {, or a } \\
\text { candle extinction jar, in a } 37^{\circ} \mathrm{C} \text { incubator }\end{array}$ \\
\hline $\begin{array}{l}\text { Exconjugant } \\
\text { Temperature }\end{array}$ & $\begin{array}{l}\text { Cultures were incubated in a } 5 \% \mathrm{CO}_{2} \\
37^{\circ} \mathrm{C} \text { incubator }\end{array}$ & $\begin{array}{l}\text { Incubation temperature for selection was } \\
\text { lowered to } 35^{\circ} \mathrm{C}\end{array}$ \\
\hline Conjugation Mutagenic Plasmid & $\begin{array}{l}\text { The pJS01 plasmid was transferred to } \\
\mathrm{S} 17-1 \text { and used in the conjugation }\end{array}$ & $\begin{array}{l}\text { As noted above, the pJSO2 plasmid was } \\
\text { constructed used for the conjugation }\end{array}$ \\
\hline Bacterial Strain & $\begin{array}{l}\text { S17-1 E. coli were prepared for } \\
\text { electroporation, and used as outlined } \\
\text { above }\end{array}$ & $\begin{array}{l}\text { Fresh S17-1 E. coli (Biomedal) were ordered } \\
\text { and used as before }\end{array}$ \\
\hline
\end{tabular}

In addition to conjugations, electroporation transformation techniques were attempted with both plasmids when no transconjugant colonies could be obtained. Electroporation protocols in $B$. henselae are frequently reported ${ }^{[235,236]}$, although as transformation by electroporation may be damaging to bacteria, conjugation was our first preference. In the Bartonella literature, most electroporation procedures are largely similar, with only minor variations in media and recovery time ${ }^{[243,244]}$. In the literature, recovery of electroporated cells was done using a supplemented RPMI broth that included rabbit blood lysate; we were not able to obtain animal blood in time for our experiments, so Schneider's Medium containing FCS was used as a substitute. 
Though wild type $B$. henselae were found to grow under these conditions, no merodiploid colonies were found using electroporation. Additionally, after the conclusion of this study, other members of our lab carried out further B. henselae electroporation using sheep blood lysate and similarly did not obtain merodiploid colonies. All variations attempted are listed in the table below.

Table 3.2. - B. henselae Electroporation Experimental Variations Variations listed in above were performed as for bi-parental transformations, in duplicate and beside the regular electroporation protocol.

\begin{tabular}{|c|c|c|}
\hline Experiment Step & Regular Protocol & Variation \\
\hline $\begin{array}{l}\text { B. henselae Culture } \\
\text { Preparation }\end{array}$ & $\begin{array}{l}\text { Two confluent } B \text {. henselae plates were suspended } \\
\text { in M199S, and then washed via centrifugation }\end{array}$ & $\begin{array}{l}\text { Either one or three confluent plates were } \\
\text { harvested and washed }\end{array}$ \\
\hline $\begin{array}{l}\text { Plasmid } \\
\text { Electroporation } \\
\text { Concentration }\end{array}$ & $\begin{array}{l}\sim 400 \mathrm{ng} \text { of total plasmid DNA was treated with drop } \\
\text { dialysis and mixed with } B \text {. henselae cells on ice for } \\
\text { electroporation }\end{array}$ & $\begin{array}{l}\sim 200,300,500,600 \mathrm{ng} \text { of total plasmid } \\
\text { DNA were used for electroporation }\end{array}$ \\
\hline $\begin{array}{l}\text { Recovery Incubation } \\
\text { Length }\end{array}$ & $\begin{array}{l}\text { Electroporated cells were immediately recovered in } \\
\text { pre-warmed } 37^{\circ} \mathrm{C} \text { Schneider's Medium containing } \\
\mathrm{FCS} \text {, and then incubated overnight in a } 5 \% \mathrm{CO}_{2}, 37^{\circ} \mathrm{C} \\
\text { incubator }\end{array}$ & $\begin{array}{l}\text { Electroporated } B . \quad \text { henselae were } \\
\text { recovered for seven hours, the streaked } \\
\text { onto selective plates }\end{array}$ \\
\hline $\begin{array}{l}\text { Recovery Incubation } \\
\mathrm{CO}_{2} \text { Conditions }\end{array}$ & $\begin{array}{l}\text { Recovered cultures were incubated overnight in a } \\
5 \% \mathrm{CO}_{2}, 37^{\circ} \mathrm{C} \text { incubator }\end{array}$ & $\begin{array}{l}\text { Cultures were sealed in either a } 5 \% \mathrm{CO}_{2} \\
\text { GENbox, a } 5 \% \mathrm{CO}_{2} \mathrm{GENbag} \text {, or a candle } \\
\text { extinction jar, in a } 37^{\circ} \mathrm{C} \text { incubator }\end{array}$ \\
\hline $\begin{array}{l}\text { Recovery Incubation } \\
\text { Temperature }\end{array}$ & $\begin{array}{l}\text { Cultures were incubated overnight in } 5 \% \mathrm{CO}_{2}, 37^{\circ} \mathrm{C} \\
\text { incubator }\end{array}$ & Cultures were incubated at $35^{\circ} \mathrm{C}$. \\
\hline $\begin{array}{l}\text { Transformation } \\
\text { Plasmid }\end{array}$ & $\begin{array}{l}\text { Electroporation was performed with purified pJSO2 } \\
\text { plasmid DNA }\end{array}$ & $\begin{array}{l}\text { pJS01 was used for electroporation with } B \text {. } \\
\text { henselae }\end{array}$ \\
\hline Bacterial Strain & $\begin{array}{l}\text { S17-1 E. coli were prepared for electroporation, and } \\
\text { used as outlined above }\end{array}$ & $\begin{array}{l}\text { Fresh S17-1 E. coli (Biomedal) were } \\
\text { ordered and used as before }\end{array}$ \\
\hline
\end{tabular}




\subsection{Discussion}

The goal of this experiment was to generate a $\Delta \operatorname{carD} B$. henselae mutant, with the aim of comparing growth of the mutant to wild-type $B$. henselae. To determine the reason why merodiploid $B$. henselae colonies were not recovered, each experimental step must be analysed.

Transformation experiments in Bartonella are commonly reported in the literature, yet despite the multiple variations tested in this study, no transformed merodiploid colonies were obtained. Due to the slowed growth rate of our S17-1 E. coli strains, we have reason to suspect that our mutagenic carD plasmids had a negative effect on our transformations.

First, regarding our conjugations, the delayed growth of S17-1 containing our pJS01 or pJS02 plasmids suggests that these plasmids interfered with normal E. coli growth; this delay may extend to other, non-essential functions as well, e.g. pilus formation. This would thus lower the chances of a successful transformation. Second, transformation of our plasmids through electroporation does not require an intermediate S17-1 E. coli host, although merodiploid colonies were still not recovered. This suggests that either $B$. henselae growth is also affected by the carD fragment, or that the electroporation failed. Although no arcing was observed during the delivery of the pulse, electroporation is known to be damaging to cells, and we were unable to obtain merodiploid colonies. Additionally, other labs have reported difficulty with Bartonella electroporation protocols (Dr Jane Koehler, personal communication).

Potential toxicity of the carD mutagenic plasmid could be explained in several ways. A mutation in the pJM05 plasmid backbone, affecting, for example, the kanamycin 
resistance gene, has previously been observed to impact growth to a similar degree to that which we have observed in our pJS02 strain (Jane Koehler, personal communication). This would explain why we observed slower growth with pJS02, compared to pJM05. However, this would not explain why we observed a slow growth rate with pJS01. Sequencing of the pJS02 plasmid, in addition to the MCS and the carD flanking region junction, would confirm if this is the case.

Alternatively, possible toxicity of the mutagenic plasmid could be due to latent expression of the truncated $c a r D$ gene. Although around $60 \%$ of the $\operatorname{carD}$ open reading frame is deleted in our plasmid constructs, expression of the 85 remaining CarD amino acids on the pJS01 sequence plasmid is possible as no frame shift was introduced. The start codon is still present, and transcription of a truncated CarD protein is possible. The pJS01 hypothetical CarD protein has an amino acid sequence that is homologous to the N-terminal region of $M$. tuberculosis CarD and M. xanthus CdnL. The N-terminus region includes the $\beta$-sheets that make up the Tudor-like RNA polymerase binding domain of the proteins; although the hypothetical $B$. henselae CarD protein is heavily truncated, it has amino acid homology to $M$. tuberculosis $\beta 1$ and $\beta 2$ folds (a possible truncated $\beta 3$ fold) and $M$. xanthus $\beta 1, \beta 2$, and $\beta 3$ folds (with a possible truncated $\beta 4$ fold (see Appendix Figure 8.1 ${ }^{[215,217]}$. As our plasmids are high copy number, even a small level of expression from each plasmid could lead to an expression sufficient to impact growth. While the target for $B$ henselae CarD is unknown, both Mycobacterium and Myxococcus CarD homologues target the $\beta$-subunit of RNA polymerase, while both B. henselae and E. coli DksA target the secondary channel of RNA polymerase $^{[209,211,219]}$. Though the target site on RNA polymerase differs between CarD and DksA, CarD fragment expression could potentially disrupt biological function in 
E. coli and B. henselae by blocking the RNA polymerase binding site of CarD or DksA, or by interfering with transcription ${ }^{[212]}$.

In conclusion, several follow-up experiments could be performed to determine why the transformations did not result in colonies, and to successfully generate a carD deletion strain. A positive transformation control reaction could be carried out, introducing a broad host range plasmid into $B$. henselae, to confirm that the transformation techniques are working. The mutagenic plasmid could be also remade, including a $\operatorname{carD}$ flanking region insert that lacks any of the $c a r D$ open reading frame and contains only intergenic sequence flanking carD. Such a plasmid would likely reduce toxicity in S17-1 by eliminating any CarD fragments, and allow formation of a merodiploid strain. This has previously been carried out for the M. xanthus $c d n L$ gene (Bartonella carD-homologue), which is an essential gene ${ }^{[245]}$. Although $M$. xanthus $c d n L$ deletion mutants were not recovered, the merodiploid strain could be constructed; subsequent negative selection for loss of the integrated plasmid resulted exclusively in restoration of the wild type for all colonies screened. Lastly, though $B$. henselae grows in a $5 \% \mathrm{CO}_{2}, 37^{\circ} \mathrm{C}$ stationary incubator, the optimum artificial conditions for bacterial growth are in a candle extinction jar and on heart infusion agar rabbit blood (HIAR) plates; these conditions could be used as the primary growth method if further experiments with $B$. henselae are carried out. 


\section{Chapter 4. Growth and Stress Response Assays}

\subsection{Introduction}

The aim of this chapter was to identify the level of stress exposure for each B. henselae stress condition to be used in our subsequent qPCR experiments.

Throughout the Bartonella life cycle, B. henselae encounters a range of environmental conditions. Though CarD has been shown to be involved in responding to specific bacterial stresses in other bacteria ${ }^{[212,223]}$, little is known about the role of $B$. henselae CarD. To investigate the role that B. henselae CarD plays in responding to specific stresses, we examined expression of carD under a range of common Bartonella stress conditions.

To accurately quantify $B$. henselae carD expression levels in response to stress, we needed to first ascertain the conditions under which the bacteria were experiencing stress but were still viable. To this end we performed stress response assays that determined the level of stress that elicits expression of stress genes while avoiding excessive cell death. This information can be compared to stress sensitivity described for other Bartonella and bacterial species, and will be used as a starting point for our carD quantification studies in B. henselae.

\subsection{1. $\quad$ Stress Conditions}

The stress conditions chosen all represent stresses that $B$. henselae is likely to encounter during infection and transmission, and a subset of these have been previously found to induce $\operatorname{carD}$ expression in other bacteria. We chose five stress conditions to test: low 
temperature, haemin exposure, ciprofloxacin treatment, hydrogen peroxide exposure, and nutrient starvation.

B. henselae has previously been shown to grow in liquid Schneider's Drosophila Medium, supplemented with $10 \%$ fetal calf serum (FCS ${ }^{[7]}$; all B. henselae stress assays, apart from the starvation assay, were performed using this medium. B. henselae cultured in Schneider's Drosophila Medium containing FCS, at $37^{\circ} \mathrm{C}$, was used as the control for B. henselae carD qPCR expression under non-stressed conditions.

Though Bartonella species grows optimally at $35-37^{\circ} \mathrm{C}$, the temperature of the mammalian host, $B$. henselae and $B$. quintana are exposed to temperatures of $28^{\circ} \mathrm{C}$ in the arthropod gut, and are known to replicate under these conditions, albeit slowly $^{[108,246]}$. Temperature has been hypothesised to be a key transcriptional regulator in adaptation to the Bartonella arthropod vector, with expression of $5 \%$ of $B$. quintana genes experiencing a change during temperature shift from $37^{\circ} \mathrm{C}$ to $28^{\circ} \mathrm{C}^{[246]}$; the $\operatorname{carD}$ homologue is moderately up-regulated in $B$. quintana at $28^{\circ} \mathrm{C}$, along with the stringent response gene $d k s A$; the stringent response has been proposed to play a role in regulating expression and survival in the arthropod vector.

Temperature is also a factor in regulating expression of $l t p A$, the carD homologue in the blood borne pathogen Borrelia burgdorferi, the causative agent of Lyme disease ${ }^{[224]}$. B. burgdorferi belongs to the Spirochaetes phylum but has a life cycle that resembles that of the alphaproteobacteria Bartonella species, alternating between a mammalian host (e.g., white-tailed deer) and a hematophagous arthropod vector (e.g., Ixodes ticks). It should be noted that LtpA is a homologue to the M. xanthus CarD protein, while Mycobacterium and Bartonella CarD are homologues of the $M$. xanthus $\mathrm{CdnL}$ protein. 
Haemin is a derivative of the haem B molecule (one of the four subunits of haemoglobin), which is derived from porphyrin IX (see Figure 4.1). Haemin is an essential nutrient for Bartonella, as a source of iron and porphryin, but is toxic at high levels ${ }^{[247-249]}$; unlike many other bacterial species, Bartonella lack de novo porphyrin or haem biosynthesis pathways, so external iron-containing porphyrin is instead scavenged from the environment ${ }^{[21]}$. Haemin is found in high concentrations in the arthropod midgut, where the mammalian erythrocytes are lysed, and low concentrations are found in the mammalian circulatory system. The bacterial response to haemin has not been fully elucidated, but $B$. quintana control the cellular use of haemin through the haemin utilisation locus and removal and sequestration of excess haemin though the haemin binding protein family. B. quintana also encodes three iron response regulators rhizobial iron regulator A (rirA), ferric uptake regulator (fur), and iron response regulator (irr) ${ }^{[230,250]}$, which may play a role in the response to haemin.

The mechanism of haemin toxicity for bacteria is not fully understood; studies indicate that the presence of haemin leads to an increase in free-radicals (due to the Fenton reaction) that can cause oxidative damage to lipids, proteins and DNA (see Figure 4.2 ${ }^{[251,252]}$. Though the haemin concentration in the arthropod midgut reaches a level that is toxic to most other bacterial species, Bartonella are unusually resistant to haemin; while the haemin utilisation locus is down-regulated at high haemin concentrations, haemin binding proteins are up-regulated by low temperature and haemin $^{[230,250]}$. B. henselae haemin binding proteins are also involved in protection against oxidative stress via $\mathrm{H}_{2} \mathrm{O}_{2}{ }^{[230,250,253-255]}$. No studies into the relationship between carD and haemin have been previously performed, to the best of our knowledge. Yet, in its role as a global transcriptional regulator and in the stringent response, it may be 
that CarD is involved in the $B$. henselae response to haemin through regulating transcription of haemin binding proteins.
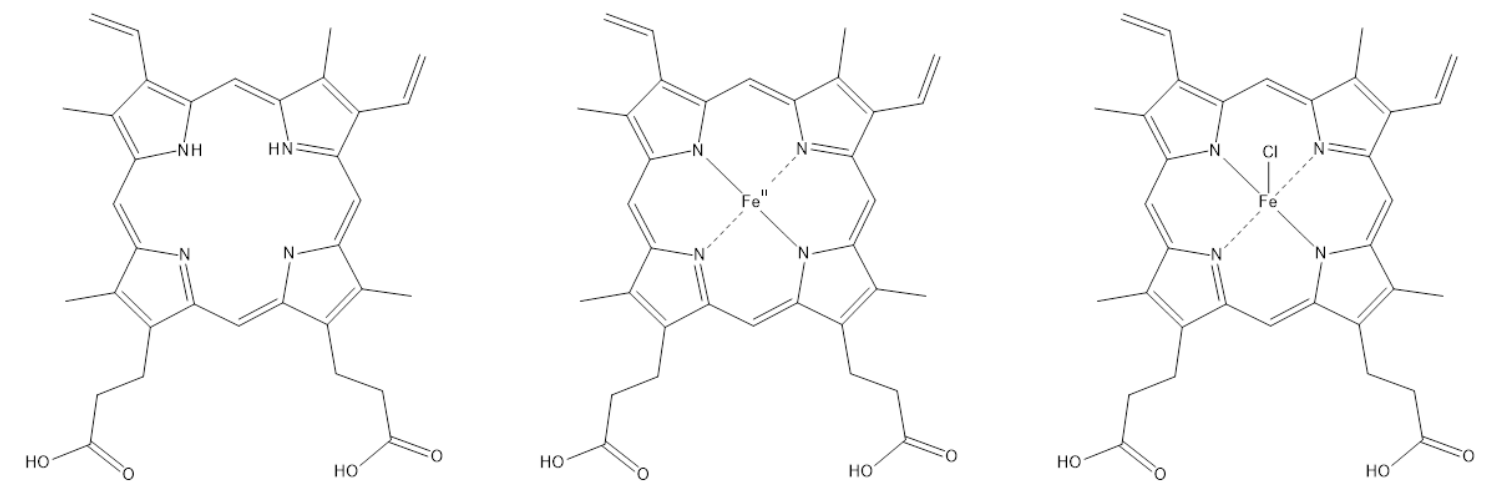

Figure 4.1. - Chemical Structure of Porphyrin IX versus Haem B versus Haemin Porphyrin IX is on the left, Heam is in the center with an $\mathrm{Fe}^{2+}$ molecule, and haemin is on the right with a chloride ion attached. Diagram was generated using ChemDraw Prime (Perkin Elmer Informatics).

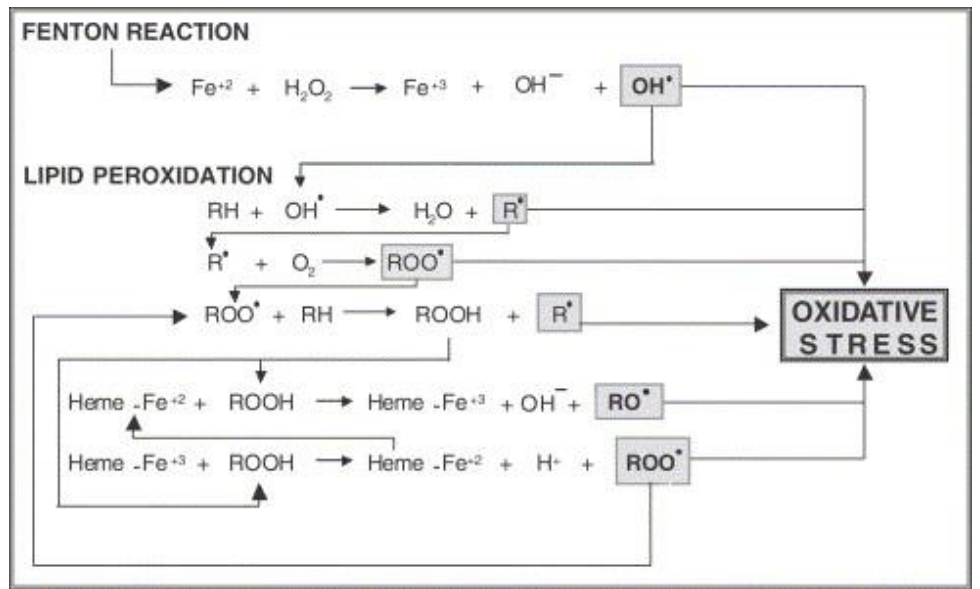

Figure 4.2. - Hypothetical Pathway of Haemin/Haem (Heme) Breakdown into Oxidative Products via the Fenton Reaction Haemin/haem is broken down via the Fention reaction to generate free radicals. Figure has been reproduced from: Insect Biochemistry and Molecular Biology, 36/4, Graça-Souza et al, Adaptations against Heme Toxicity in Blood-Feeding Arthropods, page 322, 2006, with permission from Elsevier ${ }^{[252]}$.

The antibiotic ciprofloxacin and the reactive oxygen species hydrogen peroxide $\left(\mathrm{H}_{2} \mathrm{O}_{2}\right)$ are both genotoxic agents that have been previously studied in $B$. henselae, B. bacilliformis, and in M. tuberculosi ${ }^{[212,230,253,256]}$. Ciprofloxacin is a fluoroquinolone 
class antibiotic which targets DNA topoisomerase and DNA gyrase proteins, resulting in halted transcription. Ciprofloxacin is hypothesised to release DNA ends, due to dissociation of the topoisomerase/gyrase complex from broken DNA ${ }^{[190,194,212,257]}$. Although previously used to treat Bartonella infections, it has been shown that other antibiotics are more effective ${ }^{[256,258,259]}$. Hydrogen peroxide is an important reactive oxygen species (ROS) used in cellular defence, notably by macrophage and neutrophil immune cells. As shown in Figure 4.2, $\mathrm{H}_{2} \mathrm{O}_{2}$ can be broken down into a number of oxidative agents, which cause damage to lipids, DNA, RNA, and proteins, through electron transfer ${ }^{[260]}$. Hydrogen peroxide has also been described in the arthropod midgut along with other reactive species ${ }^{[252]}$. The presence of haemin binding proteins have been described to play a role in Bartonella defence against $\mathrm{H}_{2} \mathrm{O}_{2}{ }^{[253,254]}$. In M. smegmatis, not only did the presence of both ciprofloxacin and $\mathrm{H}_{2} \mathrm{O}_{2}$ lead to an upregulation of $\operatorname{carD}$, a bacterial strain with artificially depleted $\operatorname{car} D$ had decreased resistance to both of these agents ${ }^{[212]}$.

Starvation is a distinct threat to all bacterial species, and is involved in regulation of B. henselae pathways, where the starvation-mediated stringent response plays an important role in both the arthropod vector and the mammalian host. Increased starvation conditions are likely to be encountered within the vector host midgut. Cat fleas who have previously taken a blood meal can survive for four days between blood meals, yet the majority of the protein gained during the meal will be lost after 12 hours $^{[261,262]}$; thus $B$. henselae are likely to experience a degree of nutrient deprivation inside the flea vector between meals. Along with responding to nutrient starvation, the bacterial stringent response also detects small nutritional and environmental changes to mediate transcriptional adaption to new environments. Due to the large environmental shift from the mammalian to the arthropod host, we hypothesise that carD could play 
an initial role during adaptation to the $B$. henselae arthropod vector. The stringent response is a critical mediator of bacterial adaptation to the arthropod vector of B. burgdorferi ${ }^{[263]}$. Rel ${ }_{\text {Bbu }}$ (a RelA/SpoT homologue) and (p)ppGpp levels were greatly increased during bacterial exposure to starvation conditions, and mediated a global transcriptional response to starvation in the B. burgdorferi arthropod vector.

Within the mammalian host, DksA and SpoT, two mediators of the B. henselae stringent response, have been shown to detect changes in environmental nutrients to initiate cellular invasion. In low-nutrient conditions, the up-regulation of the stringent response (DksA/SpoT) initiates VirB/D4 T4SS (mediating invasion of endothelial cells), while high-nutrient conditions down-regulate the stringent response to initiate Trw T4SS

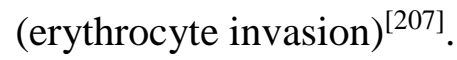

Though the role of CarD in the $B$. henselae stringent response is hypothetical, CarD directly mediates (p)ppGpp accumulation in $M$. smegmati $^{[182,212]}$; expression of the carD gene was up-regulated in response to ciprofloxacin and hydrogen peroxide, and under nutrient-deprived conditions. Transient carD depletion in M. smegmatis lowered bacterial resistance to all these conditions, including starvation ${ }^{[212]}$, and Mycobacterial CarD and E. coli DksA act in similar roles during the bacterial response to stress. We therefore decided to investigate a possible link between $\operatorname{carD}$ and the bacterial stress response in B. henselae.

\subsection{Results}

Initially our stress exposure assays were optimised with the intention of carrying out growth-rate comparisons between the wild-type and the $\Delta c a r D$ deletion strain; if $B$. henselae lacking the $c a r D$ gene had difficulty growing in a stress condition, we could infer that $c a r D$ played a role in protection against that stress. As no merodiploid $\operatorname{carD}$ 
strain was obtained, the stress assays were instead used as the foundation of our qPCR experiments, although $B$. henselae survival in the presence of stress provided important information about the bacteria. The conditions determined here may be used in future experiments to study the role of specific $B$. henselae genes in stress response.

For each stress condition, the survival and the response to the stress, described in the literature, was investigated for Bartonella and other bacterial species, to determine a starting point for our exposure assays. By applying and varying key conditions, including stressor concentration and exposure times, conditions that resulted in roughly $80 \%$ to $90 \%$ survival of the culture were determined by plating out $B$. henselae cultures and counting the number of viable colonies. We hypothesised that a survival rate of $80 \%$ to $90 \%$ would correspond to a condition where adaptive transcription responses were triggered, without excessive cell death in the population. 
B. henselae cultures grown at a temperature of $37^{\circ} \mathrm{C}$, in Schneider's Drosophila Medium containing FCS, reach a maximum $\mathrm{OD}_{600}$ of 0.5 after nine to 10 days (as shown below in Figure 4.4), at which point the bacteria reach a stationary phase where the growth rate slowed and the $\mathrm{OD}_{600}$ began to drop. By comparison, in $B$. henselae cultures at $28^{\circ} \mathrm{C}$, a slower growth rate was observed. An $\mathrm{OD}_{600}$ of 0.36 was obtained after seven to eight days. Interestingly, the initial growth at both $37^{\circ} \mathrm{C}$ and $28^{\circ} \mathrm{C}$ remained comparable for three days (reaching an $\mathrm{OD}_{600}$ of $\sim 0.29$ at $37^{\circ} \mathrm{C}$ and $\sim 0.275$ at $28^{\circ} \mathrm{C}$, at three days); deviation in the growth rate occurred subsequently. Bacterial adaptation to new conditions occurs very rapidly; therefore, for our optimised qPCR control and low temperature conditions, we assumed that an exposure time of two hours would allow for $B$. henselae to adapt to the new conditions. However, for conditions where bacterial viability rapidly decreased, shorter exposure times were used.

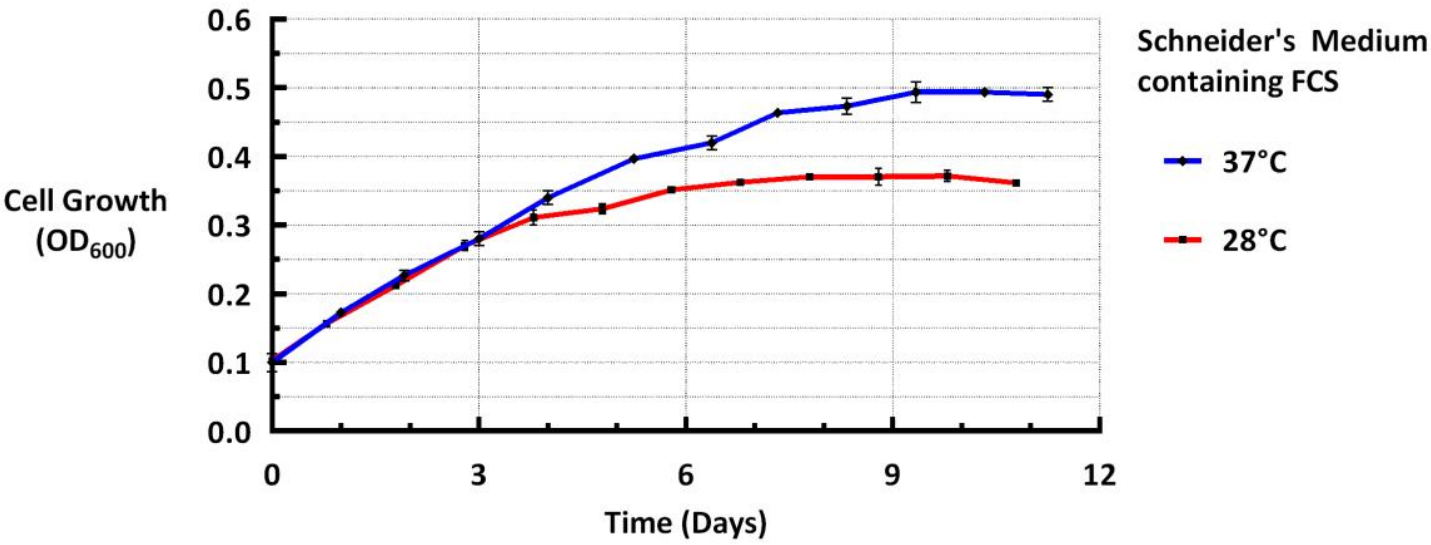

Figure 4.4. - Growth Curve of B. henselae at $37^{\circ} \mathrm{C}$ and $28^{\circ} \mathrm{C}$, Measured in $\mathrm{OD}_{600}$ over 11 Days As the majority of our stress response assays were performed in Schneider's Drosophila Medium containing FCS at $37^{\circ} \mathrm{C}$, we confirmed that the bacteria grow under these conditions and those of $28^{\circ} \mathrm{C}$. 
Haemin exposure assays were performed for haemin at a concentration of $0.1 \mathrm{mM}$ (as depicted below in Figure 4.5). A decrease in survival was observed when our B. henselae culture was exposed to $0.1 \mathrm{mM}$ of haemin. The bacteria survive exposure for up to 24 hours, though with a steady decrease in viability throughout. Initially, we cultured $B$. henselae with $0.5 \mathrm{mM}$ haemin; however, viability rapidly declined and no colonies were recovered after four hours of exposure, so a lower concentration was used.

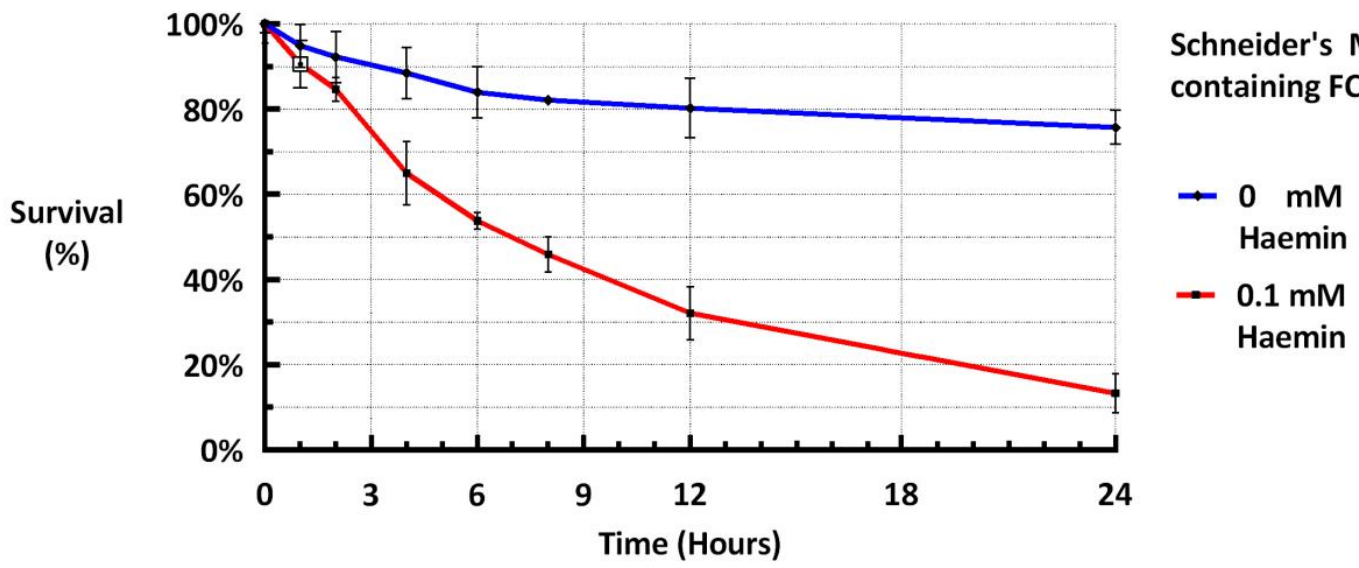

Figure 4.5. - B. henselae Haemin Stress Exposure Assay, in Percentage Survival, over 24 Hours Percentage survival was determined, first by plating dilutions of each time point, growing the plates for 14 days. The resulting colonies were counted to give the CFU, and then by comparing the CFU of each time point against the CFU of the number of initial colony forming units at a time point of $0\left(\mathrm{CFU}_{\mathrm{tx}} / \mathrm{CFU}_{\mathrm{to}} \times 100\right)$ 
Ciprofloxacin stress exposure assays were performed with two antibiotic concentrations, $10 \mu \mathrm{g} / \mathrm{mL}$ and $25 \mu \mathrm{g} / \mathrm{mL}$. A steady decrease in viability was observed at $10 \mu \mathrm{g} / \mathrm{mL}$ (as shown in Figure 4.6). The percentage survival was similar for the two concentrations of antibiotic, suggesting a degree of saturation of antibiotic within the solution. An optimum stress exposure time of $10 \mu \mathrm{g} / \mathrm{mL}$ for one hour was chosen (as denoted by an open square in our exposure assay).

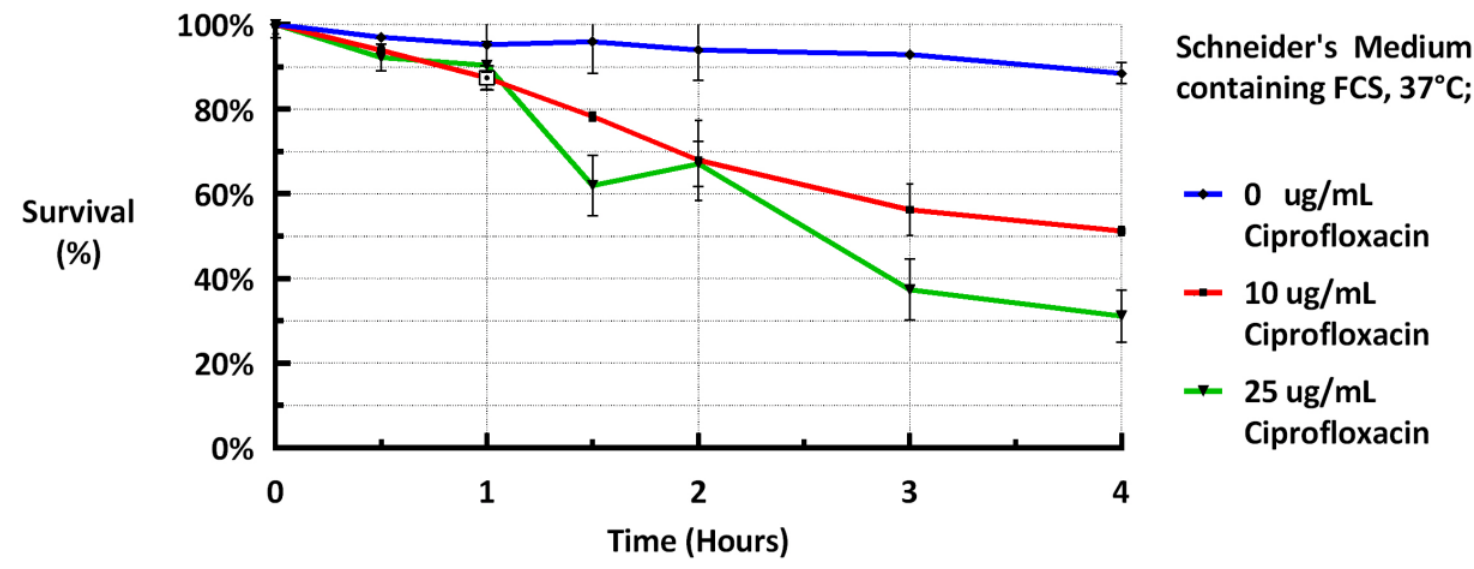

Figure 4.6. - B. henselae Ciprofloxacin Stress Exposure Assay, in Percentage Survival over Four Hours Percentage of survival relative to the initial number of colonies, was determined by exposing the bacteria over four hours. Dilutions were plated, incubated for 14 days, and then the colonies were counted as for haemin. 
Hydrogen peroxide exposure assays using 0.1 and $1 \mathrm{mM} \mathrm{H}_{2} \mathrm{O}_{2}$ were performed, as shown in Figure 4.7. At a lower concentration of $0.1 \mathrm{mM} \mathrm{H}_{2} \mathrm{O}_{2}$, viability decreased steadily over the 40 minutes of stress exposure, while at a concentration of $1 \mathrm{mM} \mathrm{H}_{2} \mathrm{O}_{2}$, a sharper decrease was observed in the first 10 minutes of exposure. Both concentrations elicited a quick drop in viability, yet a small part of the population appeared to persist at high concentrations of $\mathrm{H}_{2} \mathrm{O}_{2}$, with colonies obtained for both concentrations over the extent of our exposure assay. Exposure to $0.1 \mathrm{mM} \mathrm{H}_{2} \mathrm{O}_{2}$ for 10 minutes was chosen for our qPCR $\mathrm{H}_{2} \mathrm{O}_{2}$ stress condition (as denoted by an open square in our exposure assay).

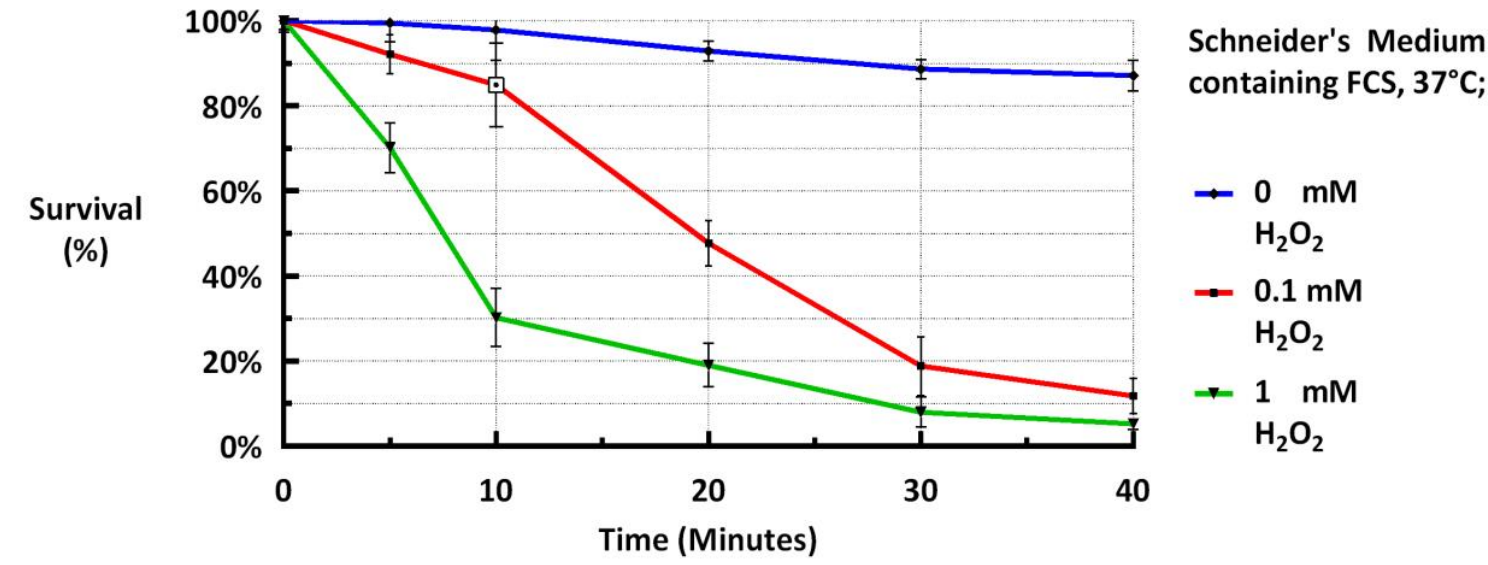

Figure 4.7. - B. henselae $\mathrm{H}_{2} \mathrm{O}_{2}$ Stress Exposure Assay, in Percentage Survival over $\mathbf{4 0}$ Minutes Percentage survival was determined by exposure bacteria over 40 minutes. Dilutions were plated, incubated for 14 days, and then the colonies were counted as for haemin. 
For our starvation exposure assay, B. henselae cultures in 1x PBS were observed to have a steady decrease in percentage survival over the time course tested, compared to the non-stressed control in Schneider's Medium containing FCS (as shown in Figure 4.8). An optimum starvation exposure time was identified of $1 \mathrm{x}$ PBS for 30 minutes at $37^{\circ} \mathrm{C}$ (as denoted by an open square in our exposure assay).

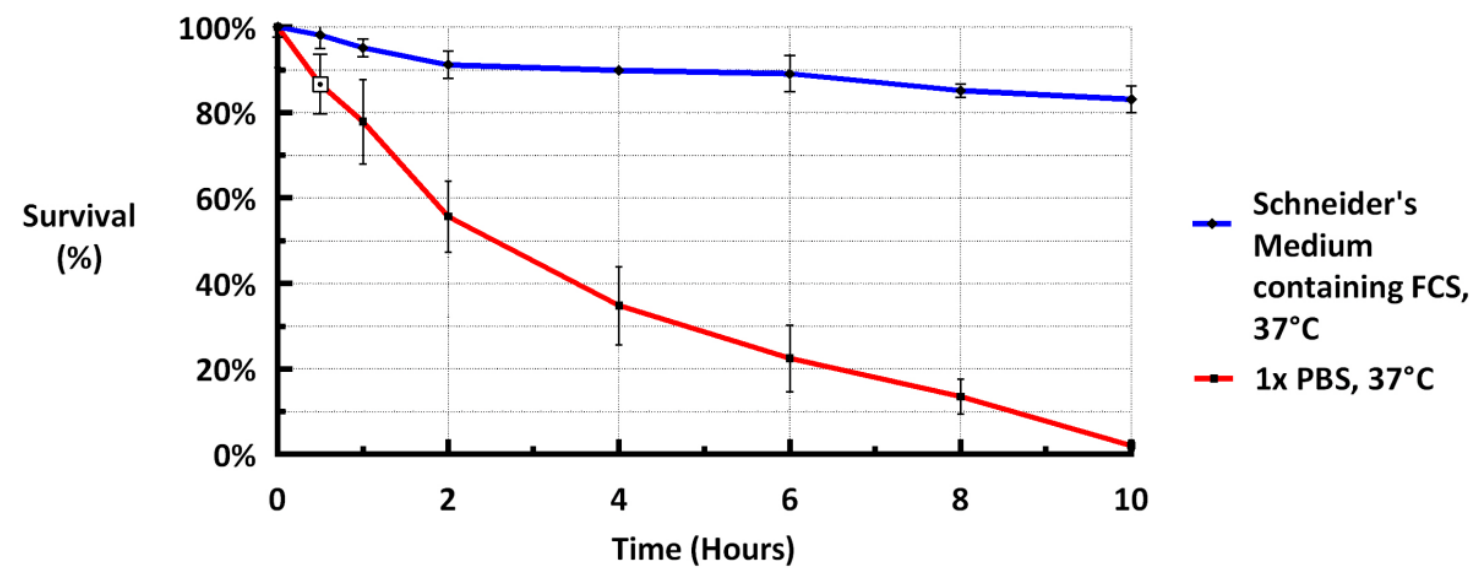

Figure 4.8. - B. henselae Starvation Stress Exposure Assay, in Percentage Survival over 10 Hours Percentage of survival was determined exposing the bacteria over a period of 10 hours. Dilutions were plated, incubated for 14 days, and then the colonies were counted as for haemin. 


\subsection{Discussion}

The overall aim of this experiment was to determine the susceptibility of $B$. henselae to a range of stresses, and to identify conditions that enabled an $80 \%$ to $90 \%$ survival rate, to test in our qPCR experiments. However, we can compare these experiments to others performed in the literature, to assess the susceptibility of $B$. henselae to each respective stress relative to other bacteria, and to consider why variations in bacterial resistance are observed.

\subsubsection{Schneider's Medium Control $\left(37^{\circ} \mathrm{C}\right)$ and Low Temperature $\left(28^{\circ} \mathrm{C}\right)$}

B. henselae has previously been shown in the literature to grow in liquid culture, in Schneider's Drosophila Medium supplemented with $10 \%$ fetal calf serum, in a $5 \% \mathrm{CO}_{2}$, $37^{\circ} \mathrm{C} 60 \mathrm{rpm}$ shaking incubator by Riess ${ }^{[7]}$. Though $B$. henselae was observed to grow in both shaking and non-shaking incubators, a faster growth rate and overall $\mathrm{OD}_{600}$ was observed with shaking (four to five days to reach an $\mathrm{OD}_{600}$ equal to 0.7 with shaking versus nine to 10 days to reach an $\mathrm{OD}_{600}$ equal to 0.5 without shaking). Despite these observations, incubation times for our stress assays did not exceed three hours (including acclimatisation time), and we therefore did not subject the cultures to shaking.

The arthropod midgut and mammalian host environments differ greatly, both in environmental stresses present and available nutrient levels; multiple transcriptional changes are required for adaptation of $B$. henselae to the appropriate host environment. Change is often mediated by the various bacterial stress responses, including the general stress response and the stringent response. 
One of the key differences between arthropod and mammalian conditions is temperature, which undoubtedly is a key mediator of adaptation of Bartonella to new host environments. $B$. henselae and $B$. quintana have been described to grow at $28^{\circ} \mathrm{C}$ within their respective arthropod vectors, although at a decreased rate compared to $37^{\circ} \mathrm{C}$ (see Figure 4.4 ${ }^{[108,246]}$; from these conditions we chose an exposure time (two hours at either $37^{\circ} \mathrm{C}$ and $28^{\circ} \mathrm{C}$ ) for our optimised qPCR stress conditions. Such a temperature shift from the mammalian host to the arthropod vector has shown to change the transcriptional profile of $B$. quintana grown at $28^{\circ} \mathrm{C}(5.0 \%$ of tested genes were temperature sensitive) ${ }^{[246]}$. Up-regulation of alternative sigma factor regulators, RpoE and PhyR (key regulators of the general stress response), and haemin binding proteins was described at $28^{\circ} \mathrm{C}^{[246,264]}$.

The increase in expression of stress response genes may be responsible for the difference in growth rate of $B$. henselae at $37^{\circ} \mathrm{C}$ and $28^{\circ} \mathrm{C}$; during the bacterial stress response, an increase in survival is often matched by a decrease in growth $^{[182,189]}$. In the B. quintana temperature transcriptional study, the change in gene expression occurred over several days, with stress gene expression increasing from day three to seven. This is an interesting trend, considering that when comparing the results of our $37^{\circ} \mathrm{C}$ and $28^{\circ} \mathrm{C}$ assays, growth only deviates after three to four days. The observed difference in B. henselae expression could be due to a delayed expression of stress genes, in a similar manner to that seen in $B$. quintana at $28^{\circ} \mathrm{C}$. Although a large difference in expression will no doubt be observed between different Bartonella species, the two studies also differ in the media used, with solid media used in the B. quintana $28^{\circ} \mathrm{C}$ study and liquid culture used in our $B$. henselae $28^{\circ} \mathrm{C}$ study. Our B. henselae RNA samples for qPCR were collected two hours after the temperature shift, to analyse the initial bacterial 
response to stress; however it would be interesting to investigate the trend of $\operatorname{carD}$ and other stress gene expression over a longer period of time.

\subsubsection{Haemin}

Along with low temperature, another key stress in the arthropod midgut is an increase in haemin concentration. Bartonella species are known to be more resistant to high haemin and haem concentrations than other bacterial species. The growth of the pathogenic bacteria Staphylococcus aureus and Haemophilus influenzae is limited in concentrations of $10 \mu \mathrm{M}$ and $0.5 \mu \mathrm{M}$ of haemin respectively ${ }^{[249,265]}$, while Porphyromonas gingivalis can survive in $50 \mu \mathrm{M}$ of haemin ${ }^{[266]}$. Reports in the literature show that haemin resistance varies among Bartonella species; B. quintana viability has been described in concentrations of $10 \mathrm{mM}$ of haemin $\left(>35.0 \%\right.$ survival at $28^{\circ} \mathrm{C}$ for 24 hours), compared to the upper bounds of $5 \mathrm{mM}$ of haemin in B. henselae $(<0.5 \%$ survival at $28^{\circ} \mathrm{C}$ for 24 hours $)^{[230,264]}$. Five haemin binding proteins are found in B. henselae, while eight are found in B. quintana ${ }^{[230,248,267]}$; differences in expression have also been described between $B$. henselae and $B$. quintana haemin binding proteins $^{[230,255]}$. Variations in the number and expression of haemin binding family members between $B$. henselae and $B$. quintana are likely due to adaptations to the respective arthropod hosts; direct comparisons between $P$. humanus and $C$. felis have not been performed, yet their feeding habits are known to differ in frequency and quantity $^{[268,269]}$.

In our haemin exposure assay, we repeatedly observed a steady decrease in growth of B. henselae, in $0.1 \mathrm{mM}$ of haemin over 24 hours (see Figure 4.5). However our results did not correspond to the levels of $B$. henselae haemin resistance reported in the 
literature; B. henselae has previously been grown on blood agar (containing $1 \mathrm{mM}$ haemin), and in Brucella broth, supplemented with 0.2 or $0.1 \mathrm{mM}$ haemin ${ }^{[247,270]}$. Another, more recent study described B. henselae (the Marseille strain, as opposed to Houston-1) growth over six days by $\mathrm{OD}_{600}$, in Borrelia media supplemented with 0 , $0.01,0.05$, and $0.1 \mathrm{mM}$ of haemin; cultures grew best at $0.05 \mathrm{mM}$, followed closely by 0.01 and $0.1 \mathrm{mM}^{[270]}$. Differences between our observed $B$. henselae response to haemin and those that have been described in the literature, could be explained by the media used. Haemin was the sole source of iron in the liquid media used by Sander et al and Muller et $\mathrm{al}^{[247,270]}$, as Brucella broth required $\geq 6 \mu \mathrm{M}$ of haemin to support bacterial growth. By contrast, Schneider's Medium containing 10\% fetal calf serum supported growth without additional iron supplementation, as the media contains free iron $(0.24 \mu \mathrm{g} / \mathrm{mL})$, haemoglobin $(<25 \mathrm{mg} / \mathrm{mL})$, and ferritin $(0.23 \mu \mathrm{g} / \mathrm{mL})$. Variations in resistance can also be seen due to differences between lab strains, protocols, and techniques. Despite the difference between $B$. henselae haemin resistance described in the literature and our results, our exposure assay demonstrated that the $B$. henselae culture was experiencing stress due to haemin, resulting in reduced viability over time; we therefore selected exposure to $0.1 \mathrm{mM}$ haemin for one hour, for analysis of $c a r D$ transcription.

For our B. henselae haemin exposure assay, decreased viability over 24 hours was assumed to be a symptom of stress. Such a death rate was thought to mimic the high level of haemin stress found in the midgut of the arthropod vector after a blood meal. However, the results of the study by Sander et al ${ }^{[247]}$ suggested a different interpretation of our results, that what we interpreted as a $B$. henselae stress response to haemin could instead be an acclimatisation period, allowing $B$. henselae to subsequently grow in the 
presence of haemin in liquid media. Sander et al grew B. henselae cultures in Brucella broth medium, supplemented with a range of haemin concentrations, and described growth by $\mathrm{OD}_{600}$ and counting viable colonies over 14 days ${ }^{[247]}$. The $\mathrm{OD}_{600}$ of B. henselae cultures grown in 0.1 and $0.2 \mathrm{mM}$ of haemin showed almost no growth for the first seven days of liquid culture, but over the next seven days, these cultures attained the highest numbers of bacteria among the tested samples. No data was provided above $0.2 \mathrm{mM}$ haemin concentration.

In our hands, a haemin concentration of $0.1 \mathrm{mM}$ results in a substantial reduction of viability in $B$. henselae, enabling us to use qPCR result to assess the $B$. henselae response to haemin. As the haemin response pathway has not been fully deduced in B. henselae, several B. quintana studies can give us some insight into this pathway. In B. quintana, an increase in haemin concentration from $0.15 \mathrm{mM}$ to $1.0 \mathrm{mM}$ induced a down-regulation in expression in all five haemin binding proteins (by roughly 2 -fold ${ }^{[255]}$. However, a further increase in haemin concentration to $2.5 \mathrm{mM}$ and $5 \mathrm{mM}$ induced an up-regulation of expression in the majority of haemin binding proteins studied (between 1.5-fold to 10-fold). Additionally, another study suggests that the opposite may be occurring with $B$. quintana haemin utilisation locus genes (involved in the uptake and use of haemin molecules $)^{[250]}$. During overexpression of different haemin response regulators, haemin utilisation locus genes were up-regulated in conditions of $0.15 \mathrm{mM}$ of haemin and down-regulated at $2.5 \mathrm{mM}$ haemin.

These values suggest that haemin concentration during an arthropod blood meal could reach levels of 2 to $5 \mathrm{mM}$. At this haemin concentration, $B$. henselae likely up-regulates haemin binding proteins and down-regulates haemin utilisation locus genes to prevent excess haemin uptake. However, between blood meals, the concentration of haemin that 
B. henselae experiences could very well fall below $1 \mathrm{mM}$, where additional haemin uptake is required, with haemin binding proteins down-regulated and the haemin utilisation locus proteins up-regulated. The level of haemin concentration we used in our haemin response assay could therefore mimic the low haemin concentration of the vector midgut during starvation. In our assay, B. henselae cultures are transitioned from regular growth in Schneider's Medium containing FCS to media with comparatively high levels of environmental haemin; this transition could be temporarily detrimental to growth of the bacteria until they adapt through gene expression and protein synthesis.

\subsubsection{Ciprofloxacin}

Ciprofloxacin has previously been used to treat Bartonella infections; however, resistance to the drug has been frequently reported in clinical Bartonella infections $^{[256,258,259]}$. The Bartonella response to ciprofloxacin and other fluoroquinolones has been assessed, using minimum inhibitory concentrations (MIC) calculated by the Etest assay ${ }^{[256,259,271]}$. The majority of fluoroquinolone resistance was judged to be the result of one of several gyrA point mutations (Asp-87 and Ser-83, using the E. coli notation); these mutations have been described in B. henselae, B. quintana, B. bacilliformis (where a single mutation increased resistance to ciprofloxacin by 16-fold), E. coli, and M. tuberculosis (multiple mutations increased ciprofloxacin resistance by $>100$-fold ${ }^{[271-275]}$. Ciprofloxacin resistant B. bacilliformis strains (gyrA) appeared after five passages with ciprofloxacin treatment, while no gentamicin or doxycycline resistant strains appeared after 16 passages.

For our initial $B$. henselae ciprofloxacin exposure assay, a lawn of bacteria was obtained at $1 \mu \mathrm{g} / \mathrm{mL}$ after an hour exposure time; the ciprofloxacin concentration was therefore 
increased to 10 and $25 \mu \mathrm{g} / \mathrm{mL}$ over several hours, at which point we observed a steady decrease in viability $(50.0 \%$ survival after four hours exposure to $10 \mu \mathrm{g} / \mathrm{mL}$; see Figure 4.6). Exposure to $10 \mu \mathrm{g}$ for one hour was judged to elicit a stress response suitable for our qPCR experiment. The ciprofloxacin concentrations used in our experiments were well above the MIC values described for $B$. henselae, but were chosen to reduce viability of the bacteria in a short time frame (hours versus days).

Ciprofloxacin stress exposure assays have previously been performed in $M$. smegmatis, comparing the wild type to a strain with reduced $\operatorname{carD}$ expression $(10 \mu \mathrm{g} / \mathrm{mL}$ for two hours); reduced growth was demonstrated for all strains, although reduced carD expression significantly reduced bacterial resistance to ciprofloxacin ${ }^{[212]}$. Mycobacterium is more resistant to ciprofloxacin than Bartonella species; M. tuberculosis has been described to have an MIC of $2 \mu \mathrm{g} / \mathrm{mL}$, compared to $0.38 \mu \mathrm{g} / \mathrm{mL}$ for B. henselae $e^{[256,276]}$.

\subsubsection{Hydrogen Peroxide}

A high level of $\mathrm{H}_{2} \mathrm{O}_{2}$ resistance of Bartonella species is to be expected considering the intracellular niches the bacteria encounter, e.g. immune cells such as macrophages and in the midgut of the arthropod ${ }^{[254,277]}$. Hydrogen peroxide exposure has previously been described for B. bacilliformis and B. henselae; B. bacilliformis is completely resistant to $\mathrm{H}_{2} \mathrm{O}_{2}$ at a concentration of $1 \mathrm{mM}$, while $B$. henselae is susceptible to $\mathrm{H}_{2} \mathrm{O}_{2}$ at concentrations of $1 \mathrm{mM}$ and $10 \mathrm{mM}^{[254,278]}$. By comparison, our B. henselae $\mathrm{H}_{2} \mathrm{O}_{2}$ exposure assay was performed for two concentrations, $0.1 \mathrm{mM}$ and $1 \mathrm{mM} \mathrm{H}_{2} \mathrm{O}_{2}$ for 30 minutes, with a rapid reduction in viability (see Figure 4.7). The discrepancy between our results and those reported for $B$. henselae in the literature are not 
insignificant; after exposing $B$. henselae cultures to $1 \mathrm{mM} \mathrm{H}_{2} \mathrm{O}_{2}$ for 30 minutes we observed a $10.0 \%$ survival rate, while Liu et al observed a roughly $50.0 \%$ survival rate after exposure to $1 \mathrm{mM} \mathrm{H}_{2} \mathrm{O}_{2}$, and a $>15.0 \%$ survival rate after $10 \mathrm{mM} \mathrm{H}_{2} \mathrm{O}_{2}$ exposure, both for 30 minutes ${ }^{[253,254]}$. Differences in $\mathrm{H}_{2} \mathrm{O}_{2}$ resistance between B. bacilliformis and B. henselae may be explained by the overall differences between the two species; however, we currently have no explanation as to why variation in $\mathrm{H}_{2} \mathrm{O}_{2}$ resistances were observed between the $B$. henselae assays performed in this study and those in the literature. Though both studies used $B$. henselae Houston-1 strains, in the literature the $\mathrm{H}_{2} \mathrm{O}_{2}$ exposure took place in 1x PBS and performed two post-exposure washes ${ }^{[254]}$, as opposed to an $\mathrm{H}_{2} \mathrm{O}_{2}$ exposure and no wash step in our study; it is possible that $\mathrm{H}_{2} \mathrm{O}_{2}$ was carried over during our dilutions, which negatively affected $B$. henselae growth. However, it is clear from both assays that $B$. henselae can survive exposure to different levels of $\mathrm{H}_{2} \mathrm{O}_{2}$, and that a stress response has been elicited in our assay.

Growth of $M$. smegmatis was not negatively impacted during exposure to $10 \mathrm{mM}$ of $\mathrm{H}_{2} \mathrm{O}_{2}$, and bacterial survival was lowered following exposure to $35 \mathrm{mM} \mathrm{H}_{2} \mathrm{O}_{2}$ (although colonies were recovered). Interestingly, M. smegmatis strains with reduced carD expression had greatly reduced growth during exposure of the culture to $\geq 10 \mathrm{mM}$ of $\mathrm{H}_{2} \mathrm{O}_{2}{ }^{[212]}$. Mycobacterial species commonly infect macrophages for extended periods, and are likely resistant to high $\mathrm{H}_{2} \mathrm{O}_{2}$ concentration, in contrast to other bacterial species $^{[197]}$. 


\subsubsection{Nutrient Starvation}

The stringent response has been shown to be crucial in bacterial adaptation to nutrient stress in innumerable bacterial species; for example, growth of Borrelia burgdorferi and Brucella suis during nutrient starvation has been previously described to require the SpoT-homologues Rel ${ }_{B b u}$ and Rsh respectively ${ }^{[263,279]}$. Similarly, B. burgdorferi Rel ${ }_{\mathrm{Bbu}}$ was also shown to mediate bacterial adaptation to its arthropod vector, the Ixodes tick, via global transcriptional regulation.

No studies have been carried out on the role of CarD in adaptation to nutrient starvation in B. henselae or other Bartonella species, to the best of our knowledge. However, the process has been studied in other bacterial species. During our starvation exposure assay, we observed a steady decrease in $B$. henselae viability, with no colonies recovered after 10 hours (see Figure 4.8). A similar $M$. smegmatis starvation exposure assay has been carried out alongside $\operatorname{carD}$ depleted strains over 24 hours ${ }^{[216]}$. A large number of M. smegmatis colonies were recovered over the entire period, while the $c a r D$ depleted strains experienced increased sensitivity to starvation.

Along with detecting and controlling bacterial survival in nutritional starvation conditions, the stringent response also detects and controls adaptations to fine changes in the environment. An example of this is the regulation of B. henselae DksA and SpoT in the mammalian host. In early host invasion, it is proposed that the decrease in nutrients in the extracellular fluid environment up-regulate DksA/SpoT, which in turn positively regulate the VirB/D4 T4SS system; in the nutrient rich bloodstream, DksA/SpoT are down-regulated, de-repressing expression of the Trw T4SS, allowing invasion of erythrocytes. Although the nutrient level in the extracellular fluid is lower than that of the bloodstream, a large number of salts, ions, proteins, and nutrients are 
still present, in contrast to the more complete nutrient deprivation in our assay; therefore B. henselae likely do not lose viability in vivo as rapidly as we observed.

\subsubsection{Conclusion}

For each of the $B$. henselae stress conditions outlined above (low temperature $\left[28^{\circ} \mathrm{C}\right]$, haemin supplementation, ciprofloxacin treatment, hydrogen peroxide exposure, and nutrient deprivation), we have tested and identified the conditions that result in viability

of $80 \%$ to $90 \%$ of $B$. henselae cells. These culture conditions were selected for PCR analysis of the $B$. henselae carD gene, to determine if the gene is regulated in response to stress.

Few studies have been carried out in B. henselae, or in other Bartonella species, for the precise stress conditions tested here. However, similar studies of the bacterial response to stress in other bacterial genera were used to approximate the resilience of $B$. henselae to stress. B. henselae stress resistance was in line with what has been previously reported, and where it differs from what was expected (i.e., haemin and hydrogen peroxide), we have provided possible explanations to explain the lower $B$. henselae stress resistance reported in this study. 


\section{Chapter 5. qPCR Analysis of carD Expression under Stress Conditions}

\subsection{Introduction}

The aim of this chapter was to investigate through $q P C R$ whether B.henselae carD expression increased in response to exposure to environmental bacterial stresses.

The overarching goal of this project was to elucidate the role that the $\operatorname{carD}$ gene plays in the $B$. henselae response to common stress conditions. Initially we sought to answer our research question through the analysis of a null mutant compared to the wild type. However, as no merodiploid or $\Delta c a r D$ mutants were obtained, we turned to quantitative PCR (qPCR) to examine the expression of $c a r D$. $\mathrm{qPCR}$ is a routine molecular biology technique to determine gene expression levels in both prokaryote and eukaryote cells. RNA can be reverse transcribed into cDNA, which acts as the template for a qPCR reaction using fluorescent DNA markers; thus the fluorescent signal of a qPCR reaction is linked to the abundance of specific transcripts. A qPCR target reaction signal can be used to calculate the relative DNA abundance, or relative gene expression, between baseline and treatment conditions. A change in relative DNA expression may suggest that the gene is up- or down-regulated in response to external stimuli.

B. henselae wild type cultures were harvested and exposed to each stress condition as described in Chapter 4 (low temperature, haemin, ciprofloxacin, $\mathrm{H}_{2} \mathrm{O}_{2}$, and starvation), then $\operatorname{car} D$ expression was analysed through qPCR; the resulting qPCR reaction signals were compared against a reference housekeeping gene and a non-stressed $B$. henselae control to calculate relative $\operatorname{carD}$ expression. 


\subsection{Results}

When running a qPCR, several assumptions are made to enable the comparison of qPCR reactions. First, we assumed that for each qPCR reaction the efficiency was $100 \%$, in that the target DNA was doubled every qPCR cycle (hence a log of 2 is used to calculate the fold change). We also assumed that the observed fluorescence in a qPCR reaction is proportional to the initial cDNA quantity. The last assumption was that the reference housekeeping gene, $r p s L$, was expressed approximately equally between each of our bacterial cultures.

To analyse gene expression by qPCR, for each condition a minimum of two qPCR reactions (with replicates and controls) were concurrently run using the same cDNA template; one qPCR reaction included primers specific to the target gene while the primers for the second reaction amplified the reference gene. Reference genes are housekeeping genes whose expression is relatively constant in the bacterial conditions being investigated; although one reference gene is commonly used, the use of multiple reference genes may improve accuracy. The reference gene used in this study was the rpsL gene, which encodes the $30 \mathrm{~S}$ ribosomal protein $\mathrm{S} 12$; this reference gene is a commonly used reference gene, and has previously been used as a control for B. henselae qPCR studies [280-282].

qPCR incorporates a fluorescent probe that is activated when bound to DNA; the level of fluorescence in each qPCR reaction is measured after each cycle, and graphed (relative fluorescent units or RFU, vs cycles) to give a sigmoidal DNA amplification curve. For each qPCR reaction, the cycle threshold $(\mathrm{Ct})$ is calculated from the reaction curve. The $\mathrm{Ct}$ is the number of cycles of qPCR taken for the RFU of a reaction to pass 
above a set threshold. The threshold level is set by the user to be above the background noise, and at the beginning of the exponential phase of DNA amplification. The $\mathrm{Ct}$ of the reference gene (or genes) is compared with the $\mathrm{Ct}$ of a target gene to give the difference in DNA abundance between the reference and target genes, a value known as $\Delta \mathrm{Ct}$ (see below for equation). The $\Delta \mathrm{Ct}$ of a baseline control (that has normal expression of the target gene) is calculated and then individually compared against the $\Delta \mathrm{Ct}$ of each condition of interest; the outcome is $\Delta \Delta \mathrm{Ct}$, which represents the change in the level of gene expression between the condition of interest and the baseline, normalised with the static expression of the housekeeping gene. Normalised target gene expression or $\Delta \Delta \mathrm{Ct}$ can be further converted into fold change in gene expression using a negative log of 2 , as a lower Ct relates to an increased level of expression.

$$
\begin{gathered}
\Delta \mathrm{Ct}=\mathrm{Ct}(\text { target gene })-\mathrm{Ct}(\text { reference gene }) \\
\Delta \Delta \mathrm{Ct}=\Delta \mathrm{Ct}(\text { treated })-\Delta \mathrm{Ct} \text { (baseline) } \\
\text { fold change }=2^{-\Delta \Delta \mathrm{Ct}}
\end{gathered}
$$

For the non-stressed condition and each stress condition (low temperature $\left[28^{\circ} \mathrm{C}\right]$, haemin, ciprofloxacin, $\mathrm{H}_{2} \mathrm{O}_{2}$, and starvation) $B$. henselae cultures were stressed with the optimum stressor level, as determined by our stress response assays. Total RNA was harvested from each culture and treated with RNAse-free DNAse, which was subsequently removed. RNA samples were then reverse transcribed to cDNA. To confirm our RNA samples are free of any gDNA contamination carried over from our RNA extraction, all cDNA samples were checked by PCR reactions with prJS03 (carD qPCR) and prJS04 (carD qPCR) primer pairs, and analysed by agarose electrophoresis gel as shown below (see Figure 5.1). Amplification of bands in the appropriate product range ( $70 \mathrm{bp})$ was confirmed, and no amplification was observed in the controls that lacked reverse transcriptase. 
a)

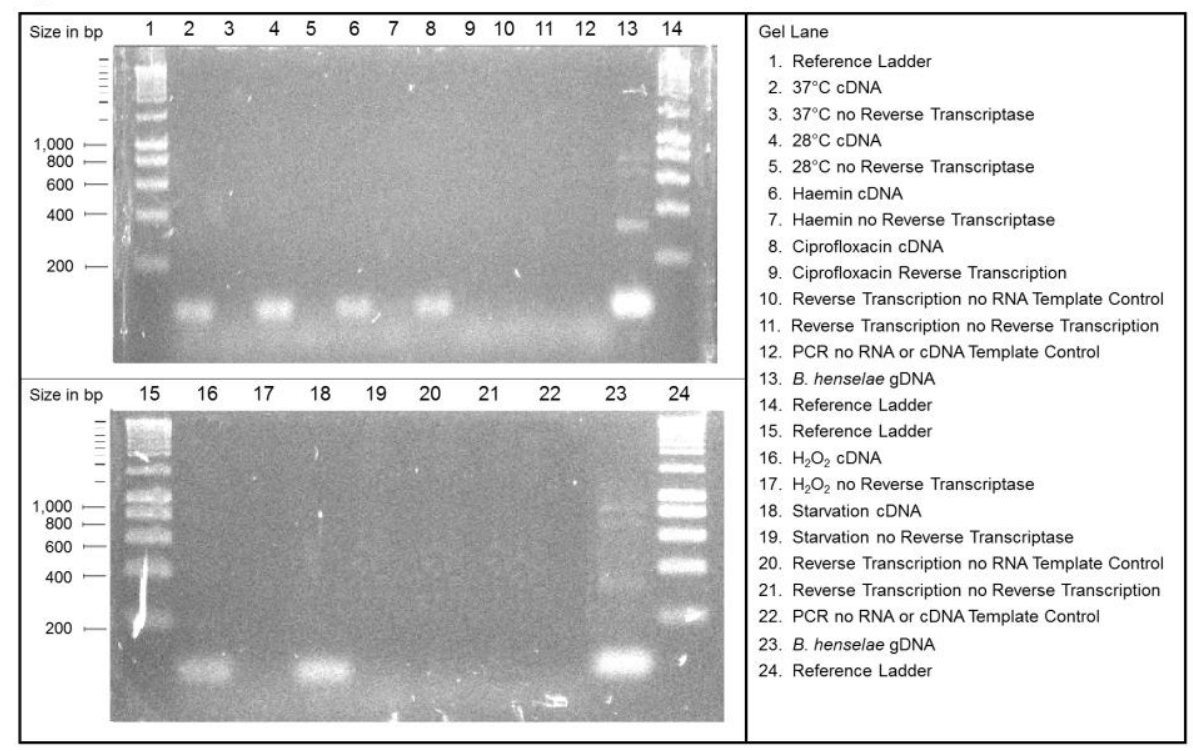

b)

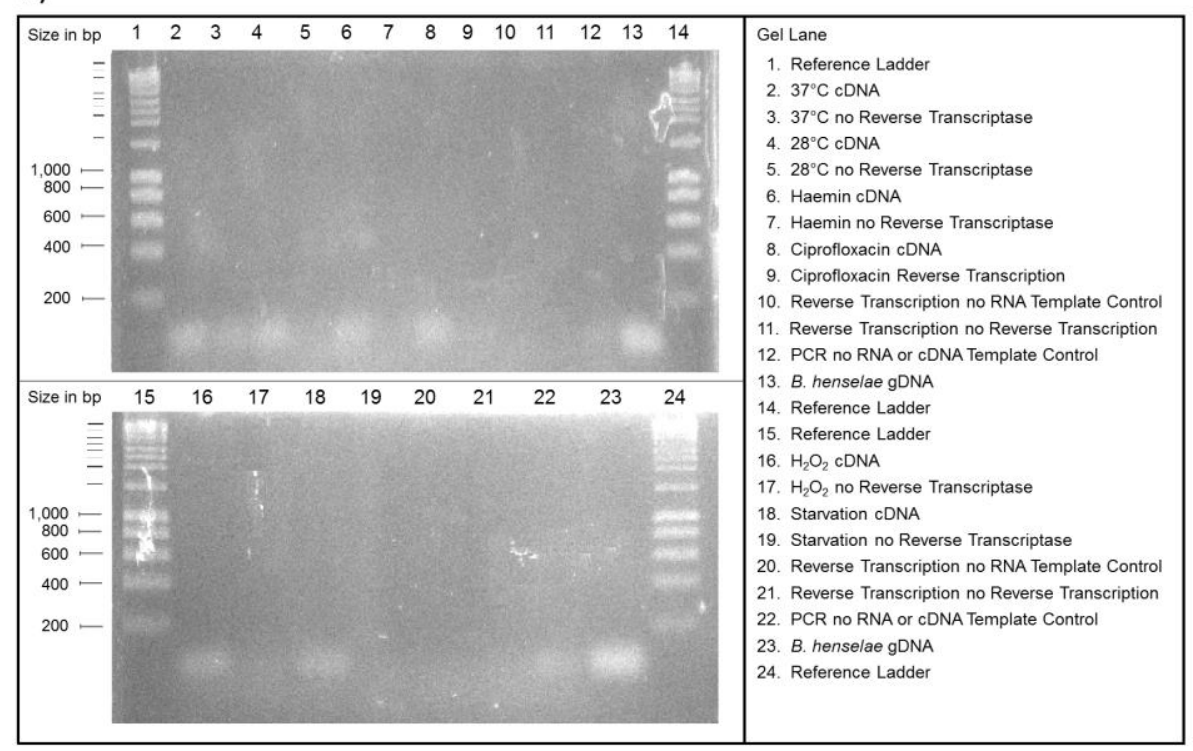

Figure 5.1. - Agarose Electrophoresis Gels of B. henselae Stress Condition Reverse Transcription Reactions To determine if our RNA extraction and our cDNA reverse transcription reaction were both successful, we performed two PCR reactions using both the prJS03 (carD) and prJS04 (rpsL) primer pairs. The cDNA of each stress condition was run beside the no-reverse transcriptase controls (to test for left over gDNA from the RNA purification), to test the reverse transcription reagents for contamination, both a no template control with no RNA added, and a no reverse transcriptase control were run. For the PCR a no cDNA template control was added to test the PCR reagents and a positive gDNA control was run for $B$. henselae. Repeats of the control lanes were run for each lane a) PCR reactions using the prJS03 (carD qPCR primer pair) were performed and run on electrophoresis gels, for each reverse transcribed stress condition to yield a 70 bp cDNA product (Lanes 2, 4, 6, 8, 16, and 18). Controls were performed alongside for the reverse transcription (no reverse transcriptase, nRT for each stress; no RNA template, RT nTC) and for the PCR (no template control, PCR nTC; $B$. henselae gDNA). b) PCR reactions were also performed using the prJS04 (rpsL qPCR primer) as for prJS03 primers. Amplification was seen as expected with the exception of $r p s L 37^{\circ} \mathrm{C} n R T$ (b, lane 3) which showed faint amplification; as no amplification was seen in the corresponding $\operatorname{carD} 37^{\circ} \mathrm{CnRT}(\mathrm{a}$, lane 3$)$, this was regarded as an anomaly. 
To test the suitability of the qPCR primers and cycle conditions, a set of qPCR experiments were performed on the $37^{\circ} \mathrm{C}$ baseline condition using the $\operatorname{prJS} 03$ (carD target gene) and prJS04 (rpsL reference gene) primer pairs, along with the respective controls $\left(37^{\circ} \mathrm{C}\right.$, no reverse transcriptase with the prJS03 and prJS04 primers and qPCR no cDNA template with prJS03 and prJS04). From this qPCR, contamination was revealed in the qPCR controls - both no reverse transcriptase control reactions and also our qPCR no template controls that lacked cDNA and RNA; this implies that the contamination has not been carried over from our initial RNA extraction. The cycle thresholds given by our initial $37^{\circ} \mathrm{C}$ qPCR reaction indicate that the level of DNA in our $37^{\circ} \mathrm{C}$ cDNA reactions was $\sim 16$ times greater than the contaminated controls before amplification through qPCR; as such, we judged that the qPCR reaction conditions and primers were viable, and we used the same qPCR cycle conditions and reagent concentrations throughout.

To eliminate the contamination, successive qPCR reactions were performed, each with fresh reagents; an unopened vial of SYBR qPCR mix was used, fresh prJS03 and prJS04 primer pairs were ordered, and PCR-grade water was used to re-suspend our primers and used in our qPCR reactions. Despite fresh reagents, PCR tubes, and tips, the contamination persisted. Along with fresh reagents, the work space and all pipettes and tools used were cleaned with $10 \%$ bleach alongside the regular $70 \%$ ethanol; care was taken to open one tube at a time to avoid spread of aerosols. Despite the care taken, the biosafety cabinet we used was not solely used for PCR and qPCR and heavily used by others; as such we cannot be sure that environmental contamination was completely eliminated. 
As contamination was observed in every single control tube, we hypothesised that it could be due to formation of primer dimers during the reaction; although the melt/dissociation curve of each qPCR reaction indicated that a single product was present that was specific to the primer pair used. To test this hypothesis, the post-qPCR amplified products were run on an electrophoresis gel; primer dimers should be a maximum of 40 base pairs, while qPCR products should be 70 to 75 base pairs. We determined that the $\mathrm{qPCR}$ product was $\sim 70$ base pairs, so primer dimers were ruled out as the cause of contamination (see Figure 5.2).

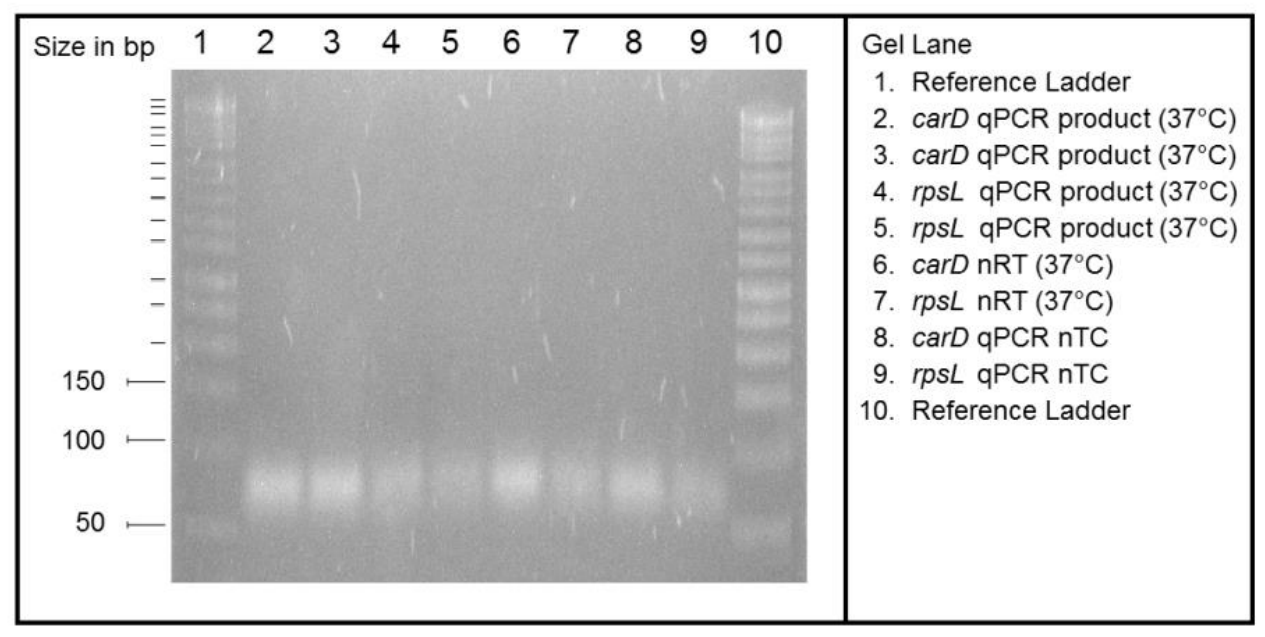

Figure 5.2. - Agarose Electrophoresis Gel of B. henselae $37^{\circ} \mathrm{C}$ qPCR Products As we saw contamination in all our lanes, we tested all our post-qPCR products for the formation of primer dimers. $5 \mu \mathrm{L}$ of each amplified qPCR lane from our initial contaminated $37^{\circ} \mathrm{C}$ qPCR reaction were run alongside a 50 base pair DNA ladder (New England Biolabs). Lanes 2 and 3 and also lanes 4 and 5 are duplicates our qPCR. As the amplified bands are $\sim 70 \mathrm{bp}$, no formation of primer dimers occurred.

If the contamination was not accounted for by the reagents or primer dimers, and was not the result of carryover of genomic DNA in the RNA samples, it was assumed to be from the environment; the most likely source of environmental contamination would be from mobile aerosols. As no other users of the qPCR machine reported contamination, 
it was likely that contamination occurred during the preparation of the qPCR reaction mixtures. Despite the extreme care taken, the contamination persisted and the source was not found; as the low level of contamination observed, with $\mathrm{Ct}$ values of $>30$ cycles (30.60 to 33.07), and taking into consideration time constraints, we decided to continue with our final qPCR experiments with the expectation that any contamination would not significantly affect final conclusions.

The $\Delta \Delta \mathrm{Ct}$ and fold change in expression were determined for each stress condition against the $37^{\circ} \mathrm{C}$ baseline control, as shown below in Figure 5.3 and in Figure 5.4. As expected, an increase in $\operatorname{carD}$ expression was observed under low temperature $\left(28^{\circ} \mathrm{C}\right)$ and starvation conditions, or in the presence of ciprofloxacin or hydrogen peroxide. In contrast, a decrease in expression was observed in the presence of haemin. Against the untreated $37^{\circ} \mathrm{C} \mathrm{B}$. henselae control, $B$. henselae $28^{\circ} \mathrm{C}$ cultures experienced a carD fold change of 1.22; the presence of haemin resulted in decreased carD expression, relative to the control condition, resulting in a fold change of $0.54 ; B$. henselae exposed to ciprofloxacin had an increase in carD expression, with a fold change of 1.27; B. henselae exposed to $\mathrm{H}_{2} \mathrm{O}_{2}$ had a fold change of 1.54; and $B$. henselae cultures exposed to starvation had a fold change, 1.27. 


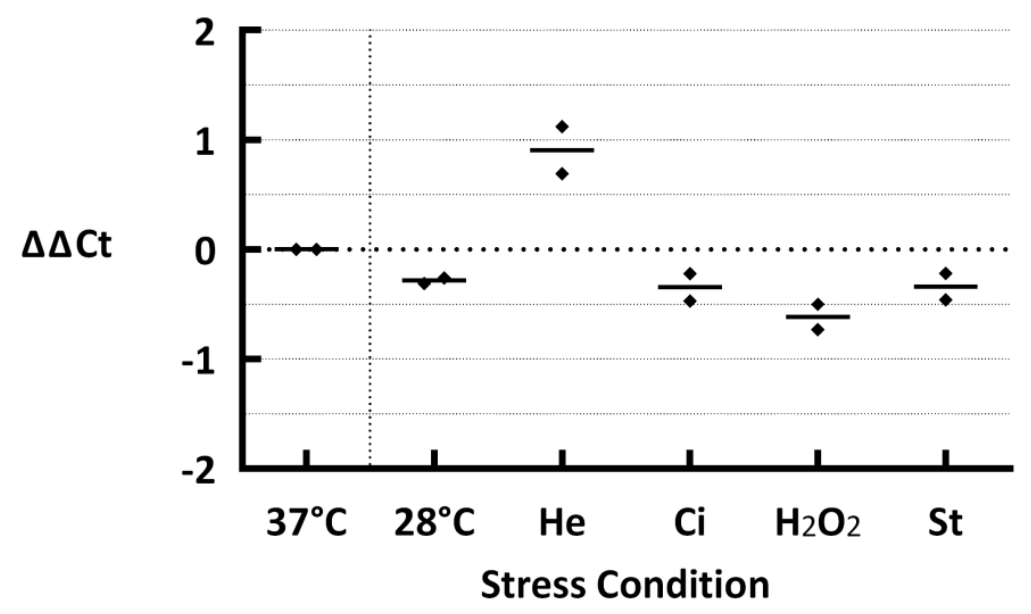

Figure 5.3. - Change in Expression $(\Delta \Delta \mathrm{Ct})$ of $c a r D$ Between $37^{\circ} \mathrm{C}$ non-stressed Control and other Stress Conditions using qPCR Duplicates of each stress condition were plotted and the mean marked with a line. $\mathrm{He}=$ Haemin, $\mathrm{Ci}=$ Ciprofloxacin, $\mathrm{H}_{2} \mathrm{O}_{2}$, and $\mathrm{St}=$ Starvation. A decrease in Ct corresponds to an increase in expression, as it indicates a greater level of initial cDNA template.

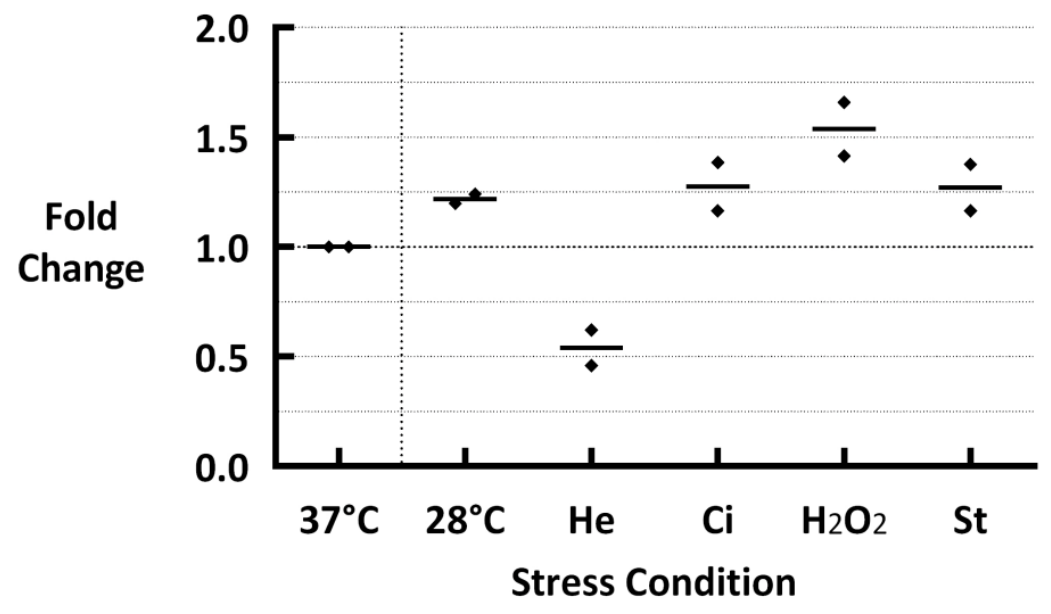

Figure 5.4. - Fold Change in Expression of carD Between $37^{\circ} \mathrm{C}$ Baseline Control and other Stress Conditions using qPCR Duplicates of each stress condition were plotted and the mean marked with a line. He = Haemin, $\mathrm{Ci}=$ Ciprofloxacin, $\mathrm{H}_{2} \mathrm{O}_{2}$, and $\mathrm{St}=$ Starvation. A fold change of 1 is equivalent to no change.

\subsection{Discussion}

The goal of this experiment was to determine the levels of expression of $c a r D$ in B. henselae in the presence of specific bacterial stresses, and to determine fold change relative to a non-stressed control, using quantitative PCR. Our results can give us an insight into $B$. henselae transcription in key environmental niches, including the 
haematophagous arthropod vector and the mammalian host. Our original hypothesis is that $c a r D$ is a stress response gene in $B$. henselae; this has been reported in other bacterial species where $\operatorname{carD}$ has been previously described (Mycobacterium carD and Myxococcus cdnL).

For each stress environment, the level of stress exposure that we used to elicit a response may not be the same level of stress that $B$. henselae would experience in its natural environment. This is largely due to the fact that it is currently not known what precise conditions the bacteria experience during the life cycle; however, by approximating conditions of stress, we can compare changes in carD transcription in response to different stress exposures.

\subsubsection{Low Temperature $\left(28^{\circ} \mathrm{C}\right)$}

For our low temperature stress exposure assay $\left(28^{\circ} \mathrm{C}\right.$ for two hours), the results of our qPCR analysis showed there to be no major change in $\operatorname{carD}$ expression relative to $37^{\circ} \mathrm{C}$ (an increase by 1.22-fold; see Figure 5.4). Though the observed fold change was minimal, the transition from $37^{\circ} \mathrm{C}$ to $28^{\circ} \mathrm{C}$ might induce greater changes in carD expression at later time points. The global B. quintana expression at $28^{\circ} \mathrm{C}$ has previously been described (through the use of a microarray) over nine days ${ }^{[246]}$. Interestingly, B. quintana carD expression at $28^{\circ} \mathrm{C}$, relative to expression at $37^{\circ} \mathrm{C}$, increased by 1.08-fold on day three, and 2.1-fold on day seven. This is similar to what we observed for our $28^{\circ} \mathrm{C} \mathrm{B}$. henselae qPCR study, suggesting that carD performs a similar role at $28^{\circ} \mathrm{C}$ for both $B$. henselae and B. quintana. The microarray also showed at $28^{\circ} \mathrm{C}$ a 4 -fold increase in expression of the general stress response gene PhyR (a regulator of RpoE), and a smaller increase in expression of the stringent response gene $d k s A$, of 0.91 -fold on day three and 2.08-fold on day seven. 
Though bacterial transcriptional changes frequently occur very rapidly in response to environmental changes, the $B$. quintana microarray study showed that stress response genes, including carD and components of the stringent response continue to be important over longer periods of time (between three to seven days). This trend is consistent with our $37^{\circ} \mathrm{C}$ and $28^{\circ} \mathrm{C}$ growth assays, where a difference in growth rate at these temperatures was observed after three to four days (Figure 4.4). Similar up-regulation of $c a r D$ and $d k s A$ in $B$. quintana could indicate that $c a r D$ plays a role in the Bartonella stringent response.

In the tick-borne mammalian pathogen, $B$. burgdorferi, the LtpA protein has been described to regulate the transcriptional profile within the arthropod vector to promote survival $^{[224]}$. The expression of this $c a r D$-like gene, $\operatorname{ltp} A$, was increased 5-fold at a $23^{\circ} \mathrm{C}$ low temperature condition, relative to a $37^{\circ} \mathrm{C}$ control. However, LptA has homology to M. xanthus CarD, as opposed to M. xanthus CdnL (like Mycobacterium and Bartonella CarD); M. xanthus CarD and CdnL share a degree of structural homology, yet although both proteins act as global regulators, the functionality was not found to be interchangeable ${ }^{[215,283]}$. We hypothesised that despite the differences between M. xanthus CarD and CdnL, B. henselae carD could perform a similar function in C. felis, as B. burgdorferi ltpA in I. scapularis. Our evidence does not support this hypothesis, although further research is needed to determine the role of $B$. henselae CarD in the arthropod host.

\subsubsection{Haemin}

During our $B$. henselae haemin $\mathrm{qPCR}$ experiments, a decrease in $c a r D$ expression (0.54-fold relative to the control without haemin; see Figure 5.4) was observed. 
Although this result suggests that carD expression is decreased in the arthropod midgut, we observed a slight increase in $\operatorname{car} D$ expression at the lower temperature associated with the arthropod.

In our $B$. henselae haemin exposure assay, results showed a large decrease in culture survival at conditions of $0.1 \mathrm{mM}$ over 24 hours, which we interpreted to be toxic stress from haemin. However, reports in the literature differ as to the toxic effects of haemin. It has been shown that high concentrations of haemin $(0.1$ and $0.2 \mathrm{mM})$ result in a loss of viability, similar to what we observed, followed by robust growth after four days ${ }^{[247]}$. Though both studies used OD $_{600}$, Sanders et al $(2000)^{[247]}$ reported an initial decrease in viable cells at both $0.1 \mathrm{mM}$ and at $0.2 \mathrm{mM}$ (the effect was more pronounced at $0.2 \mathrm{mM}$ ), but no such decrease was seen by Muller et al (2016) ${ }^{[270]}$, at $0.1 \mathrm{mM}$, although this study was conducted by assessing $\mathrm{OD}_{600}$, which does not always detect loss of viability. The interpretation of these studies is that the optimal haemin concentrations may result in an initial loss of viability, after which the bacteria recover and are able to use the higher levels of haemin as a source of iron. This makes it difficult to determine which conditions are stressful for the bacteria. One interpretation of our data is that the bacteria in Schneider's Drosophila Medium are mildly stressed, due to low levels of available iron, while the media supplemented with haemin is actually more optimal. Further studies, for example analysis of a carD deletion mutant, will be needed to determine the role that CarD plays in adaptation to iron-depleted or iron-replete conditions.

Although the role of CarD has not been described, disruption of $\operatorname{carD}$ in the rodent pathogen Bartonella birtlesii prevented bacteraemia in animal hosts, suggesting it plays a role in establishing an infection in the mammalian host ${ }^{[176]}$. CarD could play a role in regulatory control of factors known to be important for invasion, such as the type 4 
secretion systems, possibly through interactions with the stringent response. In B. henselae, the stringent response is down-regulated in the high nutrient human bloodstream; when expression of $d s k A$ and spoT is reduced, the histidine kinase component of a virulence-regulator two component system, BatS, is up-regulated, resulting in activation of Trw T4SS and invasion of erythrocytes (see Figure 1.3) ${ }^{[255]}$.

Another consideration is that the decrease in $\operatorname{carD}$ expression observed during exposure to haemin could be explained by a decrease in overall B. henselae stress. When haemin is the sole source of iron in the media, B. henselae are able to grow, after a considerable lag period ${ }^{[247]}$; Brucella broth required a minimum of $0.006 \mathrm{mM}(3.91 \mu \mathrm{g} / \mathrm{mL})$ of haemin to support $B$. henselae growth, yet each increase of haemin up to $0.2 \mathrm{mM}$ $(130.33 \mu \mathrm{g} / \mathrm{mL})$ increased growth. Although the level of iron in Schneider's Drosophila Medium with fetal calf serum is sufficient for $B$. henselae growth, it is possible that supplementation of haemin would increase $B$. henselae growth through an increase in iron availability. Schneider's Medium containing FCS has a small amount of free iron $(0.24 \mu \mathrm{g} / \mathrm{mL})$ and the iron-binding proteins haemoglobin $(<25 \mu \mathrm{g} / \mathrm{mL})$ and ferritin $(0.23 \mu \mathrm{g} / \mathrm{mL})$ provided by the fetal calf serum ${ }^{[284,285]}$. It is possible that $B$. henselae cultures in Schneider's Medium containing FCS could ultimately grow better when supplemented with $0.1 \mathrm{mM}$ of haemin ${ }^{[249,255]}$. If this is the case, $\operatorname{carD}$ expression would be lower in the low-stress condition, as has been described for other bacteria. Further testing with haemin supplementation of Schneider's Drosophila Medium FCS, and experiments with a $\Delta c a r D$ mutant, would together provide clearer answers. 


\subsubsection{Ciprofloxacin and Hydrogen Peroxide}

In our $B$. henselae $\mathrm{qPCR}$ experiments, results showed exposure to ciprofloxacin $(10 \mu \mathrm{g} / \mathrm{mL}$ for 60 minutes $)$ and hydrogen peroxide $(0.1 \mathrm{mM}$ for 10 minutes $)$ lead to a minimal change in the expression of $\operatorname{carD}$ (by 1.27-fold and 1.54-fold respectively; see Figure 5.4). The increase in expression of $c a r D$ suggests that CarD has a small role in the response to these conditions. Both ciprofloxacin and hydrogen peroxide are genotoxic agents, so the increase in carD expression is consistent with what has been reported in other bacteria.

Ciprofloxacin interferes with bacterial DNA gyrase and topoisomerase enzymes, resulting in DNA damage, while hydrogen peroxide causes damage to DNA, RNA, lipid, and protein structures via oxidisation and the formation of oxidative agents ${ }^{[257]}$. Damage to DNA can interfere with transcription, and result in stalled RNA polymerase complex. Additionally, ciprofloxacin relies on redox pumps for cellular entry, as opposed to hydrogen peroxide, which has been described in bacterial species to have a small degree of membrane permeability ${ }^{[286,287]}$. Though only a small concentration of $\mathrm{H}_{2} \mathrm{O}_{2}$ would pass into the cell, it could explain the increased $B$. henselae susceptibility to hydrogen peroxide as opposed to ciprofloxacin that we observed in our stress response assays (Figure 4.6 versus Figure 4.7). During the natural $B$. henselae life cycle, exposure to $\mathrm{H}_{2} \mathrm{O}_{2}$ is found in both the midgut of the arthropod vector and from mammalian immune cells, while ciprofloxacin is only found during drug treatment ${ }^{[252]}$.

A qPCR microarray study from Mycobacterium smegmatis ${ }^{[212]}$, a close relative of M. tuberculosis, demonstrated that exposure to ciprofloxacin ( $10 \mu \mathrm{g} / \mathrm{mL}$ for 60 minutes) and hydrogen peroxide (10 $\mathrm{mM}$ for 60 minutes) increased the expression of $c a r D$ by the 
same level, an approximate 8-fold change. We observed very little change in carD expression under comparable conditions in $B$. henselae. However, at increased $\mathrm{H}_{2} \mathrm{O}_{2}$ exposure (35 mM for 60 minutes), viable $M$. smegmatis colonies were recovered but no change in $\operatorname{carD}$ expression was observed. A large change in $\operatorname{carD}$ expression is not surprising, considering that $\operatorname{carD}$ is known to regulate the stringent response of M. smegmatis in both conditions ${ }^{[212]}$. Mycobacterium have an almost 10 -fold greater resistance to ciprofloxacin than Bartonella (MIC of $\leq 2 \mu \mathrm{g} / \mathrm{mL}$, compared to $\leq 0.38$ ), as well as a greater resistance to hydrogen peroxide, reflecting the importance of macrophages in the pathogenesis of M. tuberculosis ${ }^{[276]}$. The high levels of induction of carD in Mycobacteria, relative to what we observed in B. henselae, highlight the possibility that Bartonella CarD plays a very different role from that of Mycobacterium CarD.

\subsubsection{Nutrient Starvation}

When we exposed $B$. henselae to complete nutrient starvation in PBS, our qPCR analysis results showed essentially no major change in carD expression (an increase of 1.27-fold; see Figure 5.4). In the M. smegmatis carD qPCR study, starvation conditions (1x PBS supplemented with the detergent Tween 80 for four hours), resulted in 20 -fold increased $\operatorname{car} D$ expression ${ }^{[212]}$. Interestingly, an extended starvation exposure time (1x PBS with Tween 80 for 24 hours) showed a smaller increase in carD expression, with a 4-fold change. This is further evidence that $B$. henselae CarD is playing a role that is distinct from that reported for Mycobacterium CarD. Although it would be good to test additional time points for $B$. henselae in starvation conditions, the different roles played by CarD in two different pathogens likely reflects the many differences between 
these bacteria, including variations in pathogen biology, life cycle, host colonisation sites, and mechanisms of transmission.

\subsubsection{Conclusion}

Our initial hypothesis was that carD played a role in adaptations to stress, similar to that described for Mycobacterium CarD. However, there are many biological differences between these bacteria, and they likely experience and are adapted to different stresses; protein homologues may therefore diverge and acquire distinct functions. Additional evidence that the function of Mycobacterium CarD differs from that of Bartonella is that the former is an essential gene, while carD mutants have been noted in a rodent Bartonella species.

It was unexpected that overall our $B$. henselae stress exposure conditions resulted in a minimal increase in $\operatorname{car} D$ expression. The interesting exception to this was the response to haemin, though the decrease in expression was not expected. This result does indicate that $\operatorname{carD}$ expression is sensitive to changes in nutrient availability, though the expression was actually increased in the relatively iron-poor condition (Schneider's Drosophila Medium containing FCS). It is possible that CarD may undergo a posttranscriptional change under stress conditions. This could explain why such a small transcriptional change was found, although a change in CarD expression was observed in B. quintana studies. Additional studies are needed to shed light on whether the role of CarD is one of adaptation to nutrient limitation, or of adaptation to the potential toxicity of nutrients such as haemin.

Previous transcriptional studies in B. quintana at $37^{\circ} \mathrm{C}$ and $28^{\circ} \mathrm{C}$ gave us an insight into the role of $c a r D$. The showed similarly low levels of $\operatorname{carD}$ and $d k s A$ expression over the 
first three days, with an increase in expression of both genes observed at seven days ${ }^{\text {[264] }}$. As $c a r D$ expression is up-regulated over time, other known stringent response genes are also up-regulated. In contrast, the general stress response of B. quintana is highly up-regulated, especially PhyR. Similarly, in B. henselae cultures at low temperature, or the presence of haemin and/or hydrogen peroxide, the general stress response has been described to be up-regulated ${ }^{[230,247,252-254,264]}$.

Interaction of $B$. henselae DksA and CarD has not been shown, but reports of $\Delta d k s A$ and $\Delta c a r D$ suggest functional similarity. $d k s A$ mutants were shown to have limited endosome formation during in vitro endothelial cell invasion, possibly due to the crucial role of DksA in mediating the VirB/D4 T4SS ${ }^{[207,208]} . \Delta c a r D ~ B$. birtlesii strains were viable on lab media, yet could not establish bacteraemia in a mouse infection model ${ }^{[176]}$. If CarD also mediates $B$. henselae expression of the VirB/D4 T4SS, this could explain why the lack of $c a r D$ prevented bacteraemia in a mammalian host.

Our observed $\operatorname{carD}$ qPCR stress results are very distinct from what has been reported in Mycobacterial species for ciprofloxacin, hydrogen peroxide, and starvation ${ }^{[212]}$. This result suggests a novel role for CarD in Bartonella species, but further studies are needed to better define this role. 


\section{Chapter 6. Research Motivations, Key Findings, and Future Directions}

\subsection{Research Motivation}

$\mathrm{CarD} / \mathrm{CdnL}$ proteins have previously been characterised in the literature, most thoroughly in Mycobacterium (CarD) and Myxococcus (CdnL), where they have been reported to act as global transcriptional regulators ${ }^{[212,215,219]}$. Mycobacterial CarD functions by binding to RNA polymerase and to DNA, stabilising the open promoter complex during transcription ${ }^{[218,220,223]}$. Additionally, Mycobacterium $\mathrm{CarD}$ regulates the stringent response, stabilising the (p)ppGpp-RNA polymerase complex; this potentiates (p)ppGpp activity in a similar manner to E. coli DksA, albeit through a different RNA polymerase binding site ${ }^{[210-212]}$. Myxococcus $\mathrm{CdnL}$ similarly binds to RNA polymerase and stabilises the open promoter complex during transcription, although the protein does not bind to $\mathrm{DNA}^{[215]}$. The difference in protein function may be due to the fact that while Mycobacterium only have the CarD protein, Myxococcus have functional CdnL and DksA proteins. M. xanthus CarD and DksA proteins are essential for bacterial viability, although their functions during the Myxococcus stress response are not yet fully understood.

Bartonella, as for Myxococcus, have both DksA and CarD proteins, although neither has been shown to be essential for growth through targeted deletion. $B$. henselae DksA is a key regulator of the stringent response during mammalian host cell invasion ${ }^{[207]}$. Despite the important regulatory role that $\mathrm{CarD}$ has been shown to play in the literature, studies on Bartonella CarD structure or function have not been carried out. In a study of the rodent pathogen Bartonella birtlesii, a genetic screen was carried out to identify factors important for invasion of erythrocytes; one of the mutants identified in the initial screen 
had carD disrupted by a transposon. The B. birtlesii carD transposon mutant could not establish bacteraemia in an in vivo mouse infection model, but was not found to play a role in erythrocyte invasion ${ }^{[176]}$. This study, in combination with our own bioinformatic analyses of the $B$. henselae and $M$. smegmatis carD and $M$. xanthus $c d n L$ genes, shows that a reasonable degree of homology is found between the three bacterial genera. We therefore hypothesised that carD plays an important role in regulating Bartonella response to stress.

Our research aimed to fill an important gap in the knowledge of Bartonella biology; to this end, we aimed to investigate whether CarD played a significant role during the initial $B$. henselae response to stresses that are commonly encountered by the bacteria.

\subsection{Key Findings}

Our initial hypothesis was that $c a r D$ acted as a stress response gene in $B$. henselae, due to amino acid similarities between CarD in Bartonella and CarD in other bacterial genera. We investigated the expression levels of $\operatorname{carD}$ in the presence of common B. henselae stress conditions, including low temperature $\left(28^{\circ} \mathrm{C}\right)$, haemin supplementation, ciprofloxacin treatment, $\mathrm{H}_{2} \mathrm{O}_{2}$ exposure, and nutrient starvation.

To this end, we initially attempted to assess the role of CarD in the B. henselae bacterial stress response by comparing growth of the $B$. henselae wild type strain against a B.henselae $\Delta$ carD strain in stress conditions. However, we could generate neither the B.henselae $\Delta c a r D$ mutant strain, nor the intermediary B. henselae merodiploid carD mutant strain. An observable decrease in bacterial growth was observed for S17-1 E. coli with our mutagenic plasmids (either pJS01 or pJS02); we believe that this was due to 
toxicity of the truncated $c a r D$ gene fragment, and could explain why transformations were not successful.

Instead, for each stress condition, a quantitative PCR reaction was performed to determine the relative expression levels of the $B$. henselae carD gene, between the non-stressed control and the stress condition. Overall, our B. henselae stress exposure conditions (with the exception of haemin) showed a minimal increase in carD expression, with a trend of a small increase in $\operatorname{carD}$ expression under stress conditions. However, our qPCR results neither prove nor disprove our hypothesis that $c a r D$ is a stress gene in $B$. henselae due to the small nature of the fold change in $c a r D$ expression. It is possible that CarD plays a role in the stress response to conditions that we did not test. Similarly, the unexpected lower expression of $c a r D$ in the presence of haemin points to a role for CarD in either nutrient deprivation or detoxification of nutrients such as haemin. Additional experiments are needed to elucidate the role of CarD in B. henselae growth and response to haemin. 


\subsection{Future Directions}

\subsubsection{Continuation of our B. henselae $\Delta$ carD Experiments}

\subsubsection{Generation of a $B$. henselae $\Delta$ carD Mutant Strain}

Initial attempts to generate a $B$. henselae $\Delta$ carD mutant using the pJS01 and pJS02 mutagenic plasmids did not yield $\operatorname{carD}$ merodiploid colonies. One possible reason for this is that expression of truncated CarD protein products interfered with normal $E$. coli growth. Another possible reason is that the $c a r D$ gene is essential in B. henselae.

To elucidate the essential nature of $M$. xanthus $c d n L$, Gallego-García et al used a similar two-step merodiploid-intermediate mutation protocol as we have described in this study $^{[215]}$. To enable homologous recombination, our mutagenic carD plasmid included a small segment of our carD gene; however, the mutagenic plasmid used by Gallego-García et al included only 3' and 5' $c d n L$ intergenic flanking sequences, and no actual $c d n L$ open reading frame sequence. By redesigning our $c a r D$ mutagenic plasmid to remove any remaining $\operatorname{carD}$ sequence, we can avoid expression of the gene, and enable transformation and subsequent homologous recombination. Successful generation of the $B$. henselae carD merodiploid and $\Delta$ carD mutant strains would provide confirmation that Bartonella carD is not an essential gene.

Even if $B$. henselae carD turns out to be an essential gene, however, merodiploid colonies should still be recovered, as these isolates have one copy of the intact, wild type gene. In the case of an essential gene, however, negative selection of the merodiploid strain may only result in reversion to wild type; this was documented by Gallego-Garcia in their studies of $M$. xanthus $c d n L^{[215]}$. If $\operatorname{carD}$ did turn out to be 
essential in $B$. henselae, another approach would be to generate a plasmid that allows for controlled conditional knockdown of the carD gene, under tetracycline control, as previously described for $M$. smegmatis $\operatorname{carD} D^{[212]}$.

\subsubsection{Growth Analysis of $B$. henselae Wild-type and $\Delta$ carD Strains during Stress Exposure}

Though we observed a small increase of $c a r D$ expression in our qPCR experiments, we do not know if the $\operatorname{carD}$ gene is enabling a transcriptional change that defends $B$. henselae against stress. If a $B$. henselae $\Delta c a r D$ mutant were obtained, we could carry out our original experimental plan to determine how important the $\operatorname{carD}$ gene is in the Bartonella stress response, similar to $\operatorname{carD}$ assays performed in $M$. smegmatis $^{[212]}$. We have already determined the optimal conditions for growth of wild-type $B$. henselae cultures during exposure for each of our stresses of interest, although the purpose of these conditions are to elicit a stress response while retaining overall culture viability in the range of 80 to $90 \%$. The growth of wild-type and $\Delta c a r D$ mutant strains could be compared, using spectrophotometry and viable colony counts to estimate the percent survival at various time points.

\subsubsection{Confirmation of the B. henselae Haemin Response and Further Haemin Condition Analysis}

As discussed in Chapters 4 and 5, the haemin supplementation concentration we used for our $B$. henselae haemin exposure assay $(0.1 \mathrm{mM})$ may be too low to mimic the initial haemin concentration of the arthropod host vector during a blood meal ${ }^{[247]}$. To confirm this, the haemin resistance assay could be extended, with time points assessed for up to 14 days. If the $B$. henselae culture recovers and robust growth is confirmed, higher haemin concentrations would be needed to replicate the conditions in the arthropod gut. 
This experiment could be followed by a $c a r D$ qPCR assay to determine the stress response, as described for the other stress conditions.

To mitigate the initial loss of $B$. henselae viability following haemin exposure, the bacteria could be gradually exposed to increasing levels of haemin, to enable a gradual acclimatisation. Finally, the expression levels of $\operatorname{carD}$ could be assessed at higher, toxic concentrations of haemin.

\subsubsection{Expansion of the scope of our B. henselae carD qPCR assays}

\subsubsection{Targeting an Increased Range of $B$. henselae Stress Genes}

We cannot conclude that the $B$. henselae carD gene is involved in the Bartonella stress response from our carD qPCR assays. To better determine the role of $c a r D$ in the stress conditions, we could also assess the expression of $B$. henselae genes that are known components of the stress response. qPCR primers can be designed that amplify the B. henselae dksA (or spoT) for the stringent response and phyR (or rpoE) for the general stress response; when used in conjugation with our carD primers, we could use these to compare the $\operatorname{car} D$ response against defined stress genes.

\subsubsection{Increase the Scope of our B. henselae carD qPCR Stress Assay}

During culture of $B$. quintana in $28^{\circ} \mathrm{C}$ (against a $37^{\circ} \mathrm{C}$ control) ${ }^{[264]}$, the initial fold change of $\operatorname{carD}$ and $d k s A$ gene expression was low after three days ( $<1.5$-fold), yet an increased fold change was observed after seven days of exposure to $28^{\circ} \mathrm{C}$ (>2-fold). The B. quintana $28^{\circ} \mathrm{C}$ study concluded that RpoE (a general stress sigma factor) played a role in temperature mediated adaptation to the arthropod vector ${ }^{[264]}$. Additionally, the 
B. henselae stress response described for haemin and $\mathrm{H}_{2} \mathrm{O}_{2}$ resulted in an increase in PhyR expression, highlighting the role of the general stress response in the initial exposure to stress in Bartonella ${ }^{[230,247,252-254,264]}$, while the stringent response (possibly through $(a r D)$ may mediate later adaptation to stress.

We may have observed a small $B$. henselae carD fold change in our qPCR studies because CarD plays a role in later stress adaptation. Increasing the exposure time of each stress response assay and measuring $\operatorname{carD}$ expression over a time course would verify whether this trend holds true in B. henselae and for multiple stresses.

\subsubsection{Testing Stress Conditions that Mimic the B. henselae Environment}

If $\operatorname{car} D$ expression changes are detected during our modified qPCR stress experiments, follow up experiments combining multiple stress signals could be carried out. For example, to mimic the arthropod gut, we could simultaneously test haemin exposure and $28^{\circ} \mathrm{C}$, including individual stress assays as controls. It would also be interesting to see if a similar carD response to starvation is found in defined media, as opposed to the complete nutrient starvation assay as described here; minimal supplemented media might better replicate the natural conditions encountered by $B$. henselae.

\subsubsection{Studies into the Role of carD during Regular Cellular Function}

Often bacterial stress genes play important roles in mediating the regular bacterial growth cycle. This has been observed for $d k s A$ in $E$. coli ${ }^{[288]}$, and in $c d n L$ in M. xanthus $^{[245]}$. It would be interesting to see if carD plays an similar role in B. henselae.

To determine this, we could attempt to quantify the expression levels of $c a r D$ in B. henselae over time in culture, by investigating the levels of the gene in wild-type 
cultures as they progress through the cell cycle. However, while we could attempt to use $B$. henselae as a model for this kind of cell cycle research, few genetic tools currently exist for Bartonella species.

Caulobacter crescentus is a free-living, Gram-negative alphaproteobacteria that lives in aquatic environments, and has CarD and DksA homologues, although their functional role has not been elucidated. C. crescentus undergoes asymmetric cellular division, resulting in two distinct cellular morphologies: a sessile mother cell with a stalk and a mobile daughter cell with a flagellum ${ }^{[289,290]}$. This allows cell cycle synchronisation, and accurate determination of the timing of gene expression; stalk cells are in $\mathrm{S}$ phase, flagellated cells in G1, and the dividing cells in G2. The organism is also highly genetically tractable, with a large number of molecular tools available. The role of the endogenous $C$. crescentus carD gene in the cell cycle could be analysed by gene deletion and mutant analysis. Finally, cross-complementation studies could be carried out between the $B$. henselae carD and $C$. crescentus, to determine if the $B$. henselae carD gene can functionally replace the $C$. crescentus carD gene. Research tools, such as transcriptional profiling or protein localisation, are available for $C$. crescentus, and these could be used to carry out a detailed analysis of the role of $B$. henselae carD during growth, the cell cycle, and stress response. 


\subsection{Concluding Remarks}

Though CarD has been well-studied in other bacterial genera, the experiments reported here are the first experimental analysis that solely focuses on the CarD in any Bartonella species.

We observed through quantitative PCR reactions a trend of increased expression of $\operatorname{carD}$ in our $B$. henselae cultures under stress conditions, although this change was not found to be substantial. Further experimentation is underway, to shed light on the role of $\operatorname{carD}$ in the Bartonella stress response. 


\section{Chapter 7. References}

1. Jones K, Patel N, et al., Global trends in emerging infectious diseases. Nature, 2008. 451(7181): p. 990-93.

2. Woolhouse M, Haydon D, et al., Emerging pathogens: the epidemiology and evolution of species jumps. Trends in Ecology \& Evolution, 2005. 20(5): p. 238-44.

3. Minnick M and Anderson B, Chapter 105 - Bartonella, 1911-1939. In Schwartzman YWTSLP (ed.). Molecular Medical Microbiology (Second Edition) Academic Press, Boston, 2015.

4. Breitschwerdt E and Kordick D, Bartonella infection in animals: carriership, reservoir potential, pathogenicity, and zoonotic potential for human infection. Clin Microbiol Rev, 2000. 13(3): p. 428-38.

5. Breitschwerdt E, Bartonellosis: one health perspectives for an emerging infectious disease. ILAR journal, 2014. 55(1): p. 46-58.

6. Brenner DJ, Hollis DG, et al., Proposal of Afipia gen. nov., with Afipia felis sp. nov. (formerly the cat scratch disease bacillus), Afipia clevelandensis sp. nov. (formerly the Cleveland Clinic Foundation strain), Afipia broomeae sp. nov., and three unnamed genospecies. Journal of Clinical Microbiology, 1991. 29(11): p. 2450-60.

7. Riess T, Dietrich F, et al., Analysis of a Novel Insect Cell Culture Medium-Based Growth Medium for Bartonella Species. Applied and Environmental Microbiology, 2008. 74(16): p. 5224-27.

8. Maggi RG, Duncan AW, et al., Novel Chemically Modified Liquid Medium That Will Support the Growth of Seven Bartonella Species. Journal of Clinical Microbiology, 2005. 43(6): p. 2651-55.

9. Maggi RG, Mascarelli PE, et al., Bartonella spp. bacteremia in high-risk immunocompetent patients. Diagn Microbiol Infect Dis, 2011. 71(4): p. 430-7.

10. Kordick DL and Breitschwerdt EB, Persistent infection of pets within a household with three Bartonella species. Emerging Infectious Diseases, 1998. 4(2): p. 325-28.

11. Chomel BB, Kasten RW, et al., Clinical Impact of Persistent Bartonella Bacteremia in Humans and Animals. Ann N Y Acad Sci, 2003. 990(1): p. 267-78.

12. Brouqui $\mathbf{P}$, Lascola $\mathbf{B}$, et al., Chronic Bartonella quintana bacteremia in homeless patients. N Engl J Med, 1999. 340(3): p. 184-9.

13. Glaus $\mathrm{T}$, Hofmann-Lehmann $\mathbf{R}$, et al., Seroprevalence of Bartonella henselae infection and correlation with disease status in cats in Switzerland. J Clin Microbiol, 1997. 35(11): p. 2883-5.

14. Chomel BB, Kasten RW, et al., Bartonella Infection in Domestic Cats and Wild Felids. Ann N Y Acad Sci, 2006. 1078(1): p. 410-15.

15. La Scola B and Raoult D, Culture of Bartonella quintana and Bartonella henselae from human samples: a 5-year experience (1993 to 1998). J Clin Microbiol, 1999. 37(6): p. 1899-905.

16. J. M. Rolain PB, J. E. Koehler, C. Maguina, M. J. Dolan and D. Raoult, Recommendations for Treatment of Human Infections Caused by Bartonella Species. Antimicrobial Agents and Chemotherapy, 2004. 48(6): p. 1921-33.

17. Maurin M, Birtles R, et al., Current knowledge of Bartonella species. Eur J Clin Microbiol Infect Dis, 1997. 16(7): p. 487-506.

18. Dehio C, Molecular and cellular basis of bartonella pathogenesis. Annu Rev Microbiol, 2004. 58: p. 365-90. 
19. Harms A and Dehio C, Intruders below the radar: molecular pathogenesis of Bartonella spp. Clin Microbiol Rev, 2012. 25(1): p. 42-78.

20. Kittler R, Kayser M, et al., Molecular evolution of Pediculus humanus and the origin of clothing. Curr Biol, 2003. 13(16): p. 1414-7.

21. Alsmark CM, Frank AC, et al., The louse-borne human pathogen Bartonella quintana is a genomic derivative of the zoonotic agent Bartonella henselae. Proc Natl Acad Sci U S A, 2004. 101(26): p. 9716-21.

22. Drancourt M, Tran-Hung L, et al., Bartonella quintana in a 4000-year-old human tooth. J Infect Dis, 2005. 191(4): p. 607-11.

23. Graham JHP, A Note On A Relapsing Febrile Illness of Unknown Origin. The Lancet, 1915. 186(4804): p. 703-04.

24. Hunt GH and Rankin AC, Intermittent Fever of Obsucre Origin, Occuring Among British Soldiers In France. The Lancet, 1915. 186(4812): p. 1133-36.

25. Mooser H, Leemann A, et al., Beobachtungen an Fünftagefieber. Pathobiology, 1948. 11(5): p. 513-22.

26. Atenstaedt RL, Trench fever: the British medical response in the Great War. Journal of the Royal Society of Medicine, 2006. 99(11): p. 564-68.

27. Hunt GH and Mcnee JW, Further Observations on 'Trench Fever': A Relapsing Fever Occurring with the British Troops in France. QJM, 1916. os-9(36): p. 442-49.

28. Töpfer H, Zur Aetiologie des "Febris Wolhynica." Berlin klin Wochschr, 1916(53, ): p. 323.

29. Arkwright JA and Duncan FM, Preliminary Note on the Association of Rickettsia Bodies in Lice with Trench Fever. British Medical Journal, 1918. 2(3012): p. 307-09.

30. Strong RP, Swift HF, et al., Trench fever: Report of commission, Medical research committee, American Red cross. 1918.

31. Raoult D, Ndihokubwayo JB, et al., Outbreak of epidemic typhus associated with trench fever in Burundi. Lancet, 1998. 352(9125): p. 353-8.

32. Ahmed MA, Abdel Wahab SM, et al., Louse-borne relapsing fever in the Sudan. $A$ historical review and a clinico-pathological study. Tropical and geographical medicine, 1980. 32(2): p. 106-11.

33. Rydkina EB, Roux V, et al., Bartonella quintana in body lice collected from homeless persons in Russia. Emerg Infect Dis, 1999. 5(1): p. 176-8.

34. Stein A and Raoult D, Return of trench fever. The Lancet, 1995. 345(8947): p. 450-51.

35. Brouqui $\mathbf{P}$, Houpikian $\mathbf{P}$, et al., Survey of the seroprevalence of Bartonella quintana in homeless people. Clin Infect Dis, 1996. 23(4): p. 756-9.

36. Jackson LA, Spach DH, et al., Seroprevalence to Bartonella quintana among patients at a community clinic in downtown Seattle. J Infect Dis, 1996. 173(4): p. 1023-6.

37. Comer JA, Diaz T, et al., Evidence of rodent-associated Bartonella and Rickettsia infections among intravenous drug users from Central and East Harlem, New York City. Am J Trop Med Hyg, 2001. 65(6): p. 855-60.

38. Comer JA, Flynn C, et al., Antibodies to Bartonella species in inner-city intravenous drug users in Baltimore, Md. Arch Intern Med, 1996. 156(21): p. 2491-5.

39. Foucault C, Barrau K, et al., Bartonella quintana Bacteremia among Homeless People. Clin Infect Dis, 2002. 35(6): p. 684-9.

40. Drancourt M, Mainardi JL, et al., Bartonella (Rochalimaea) quintana Endocarditis in Three Homeless Men. New England Journal of Medicine, 1995. 332(7): p. 419-23.

41. Schulein R, Seubert A, et al., Invasion and Persistent Intracellular Colonization of Erythrocytes: A Unique Parasitic Strategy of the Emerging Pathogen Bartonella. J Exp Med, 2001. 193(9): p. 1077-86.

42. Pitassi LH, Magalhaes RF, et al., Bartonella henselae infects human erythrocytes. Ultrastruct Pathol, 2007. 31(6): p. 369-72. 
43. Department of Health and Human Services US, The 2011 National Blood Collection and Utilization Survey Report. Washington, DC: U.S. Department of Health and Human Services, Office of the Assistant Secretary for Health; 2011.

44. Magalhaes RF, Pitassi LH, et al., Bartonella henselae survives after the storage period of red blood cell units: is it transmissible by transfusion? Transfus Med, 2008. 18(5): p. 287-91.

45. D'alessandro A, Liumbruno G, et al., Red blood cell storage: the story so far. Blood Transfusion, 2010. 8(2): p. 82-88.

46. Lin JW, Chen CM, et al., Unknown fever and back pain caused by Bartonella henselae in a veterinarian after a needle puncture: a case report and literature review. Vector Borne Zoonotic Dis, 2011. 11(5): p. 589-91.

47. Oliveira AM, Maggi RG, et al., Suspected Needle Stick Transmission of Bartonella vinsonii subspecies berkhoffii to a Veterinarian. Journal of Veterinary Internal Medicine, 2010. 24(5): p. 1229-32.

48. Silva MN, Vieira-Damiani G, et al., Bartonella henselae transmission by blood transfusion in mice. Transfusion, 2016.

49. Kordick DL and Breitschwerdt EB, Relapsing bacteremia after blood transmission of Bartonella henselae to cats. Am J Vet Res, 1997. 58(5): p. 492-7.

50. Abbott RC, Chomel BB, et al., Experimental and natural infection with Bartonella henselae in domestic cats. Comp Immunol Microbiol Infect Dis, 1997. 20(1): p. 41-51.

51. Angelakis $\mathrm{E}$, Lepidi $\mathrm{H}$, et al., Human Case of Bartonella alsatica Lymphadenitis. Emerging Infectious Diseases, 2008. 14(12): p. 1951-53.

52. Chamberlin J, Laughlin LW, et al., Epidemiology of Endemic Bartonella bacilliformis: $A$ Prospective Cohort Study in a Peruvian Mountain Valley Community. Journal of Infectious Diseases, 2002. 186(7): p. 983-90.

53. Kordick DL, Hilyard EJ, et al., Bartonella clarridgeiae, a newly recognized zoonotic pathogen causing inoculation papules, fever, and lymphadenopathy (cat scratch disease). J Clin Microbiol, 1997. 35(7): p. 1813-8.

54. Muriel V-T, Sara M, et al., Identification of Novel Zoonotic Activity of Bartonella spp., France. Emerging Infectious Disease journal, 2016. 22(3): p. 457.

55. Sofer S, Gutierrez R, et al., Molecular detection of zoonotic bartonellae (B. henselae, B. elizabethae and B. rochalimae) in fleas collected from dogs in Israel. Med Vet Entomol, 2015. 29(3): p. 344-8.

56. Boulouis $\mathrm{HJ}$, Chang CC, et al., Factors associated with the rapid emergence of zoonotic Bartonella infections. Vet Res, 2005. 36(3): p. 383-410.

57. Maggi RG, Kosoy M, et al., Isolation of Candidatus Bartonella melophagi from human blood. Emerg Infect Dis, 2009. 15(1): p. 66-8.

58. Brouqui P and Raoult D, Arthropod-borne diseases in homeless. Ann N Y Acad Sci, 2006. 1078: p. 223-35.

59. Kosoy $\mathbf{M}$, Bai $\mathbf{Y}$, et al., Identification of Bartonella infections in febrile human patients from Thailand and their potential animal reservoirs. Am J Trop Med Hyg, 2010. 82(6): p. 1140-5.

60. Schmidt S, Essbauer SS, et al., Multiple Infections of Rodents with Zoonotic Pathogens in Austria. Vector Borne and Zoonotic Diseases, 2014. 14(7): p. 467-75.

61. Kosoy $\mathbf{M}$, Murray $\mathbf{M}$, et al., Bartonella strains from ground squirrels are identical to Bartonella washoensis isolated from a human patient. J Clin Microbiol, 2003. 41(2): p. 645-50.

62. Mullins KE, Hang J, et al., Molecular typing of "Candidatus Bartonella ancashi," a new human pathogen causing verruga peruana. J Clin Microbiol, 2013. 51(11): p. 3865-8. 
63. Veikkolainen V, Vesterinen EJ, et al., Bats as Reservoir Hosts of Human Bacterial Pathogen, Bartonella mayotimonensis. Emerging Infectious Diseases, 2014. 20(6): p. 960-67.

64. Kim Y-s, Seo K-w, et al., Prevalence of Bartonella henselae and Bartonella clarridgeiae in cats and dogs in Korea. Journal of Veterinary Science, 2009. 10(1): p. 85-87.

65. Oskouizadeh $\mathrm{K}$, Zahraei-Salehi $\mathrm{T}$, et al., Detection of Bartonella henselae in domestic cats' saliva. Iranian Journal of Microbiology, 2010. 2(2): p. 80-84.

66. Jendro MC, Weber G, et al., Reactive arthritis after cat bit: a rare manifestation of cat scratch disease--case report and overview. Zeitschrift für Rheumatologie / Z Rheumatol, 1998. 57(3): p. 159-63.

67. Yoshida H, Kusaba N, et al., Clinical analysis of cat scratch disease. Kansenshogaku Zasshi / The Journal of the Japanese Association for Infectious Diseases, 2010. 84(3): p. 292-5.

68. Oshima $\mathbf{Y}$, Fujii $\mathbf{M}$, et al., Bartonella henselae infection caused by cat flea bite. Pathology International, 2016. 66(3): p. 177-79.

69. Maggi RG, Harms CA, et al., Bartonella henselae in Porpoise Blood. Emerging Infectious Diseases, 2005. 11(12): p. 1894-98.

70. Palmero J, Pusterla N, et al., Experimental infection of horses with Bartonella henselae and Bartonella bovis. J Vet Intern Med, 2012. 26(2): p. 377-83.

71. Carithers HA, Cat-scratch disease; notes on its history. Am J Dis Child, 1970. 119(3): p. 200-3.

72. Debre R, La maladie des griffes de chat. Semin Hop Paris, 1950. 26: p. 1895-901.

73. Greer WER and Keefer CS, Cat-Scratch Fever. New England Journal of Medicine, 1951. 244(15): p. 545-48.

74. Fowler RS and Bailey JD, Cat Scratch Disease in Childhood. Canadian Medical Association Journal, 1961. 84(24): p. 1365-68.

75. Daniels WB and Macmurray FG, Cat Scratch Disease. A Report of 160 Cases. Transactions of the American Clinical and Climatological Association, 1954. 65: p. 99115.

76. Carithers HA, Cat-scratch disease. An overview based on a study of 1,200 patients. Am J Dis Child, 1985. 139(11): p. 1124-33.

77. Sweeney VP and Drance SM, Optic neuritis and compressive neuropathy associated with cat scratch disease. Canadian Medical Association Journal, 1970. 103(13): p. 1380-81.

78. Leboit PE, Berger TG, et al., Epithelioid haemangioma-like vascular proliferation in AIDS: manifestation of cat scratch disease bacillus infection? Lancet, 1988. 1(8592): p. 960-3.

79. Wear DJ, Margileth AM, et al., Cat scratch disease: a bacterial infection. Science, 1983. 221(4618): p. 1403-05.

80. Relman DA, Loutit JS, et al., The agent of bacillary angiomatosis. An approach to the identification of uncultured pathogens. N Engl J Med, 1990. 323(23): p. 1573-80.

81. Perkocha LA, Geaghan SM, et al., Clinical and pathological features of bacillary peliosis hepatis in association with human immunodeficiency virus infection. N Engl J Med, 1990. 323(23): p. 1581-6.

82. Slater LN, Welch DF, et al., A newly recognized fastidious gram-negative pathogen as a cause of fever and bacteremia. N Engl J Med, 1990. 323(23): p. 1587-93.

83. Regnery RL, Olson JG, et al., Serological response to "Rochalimaea henselae" antigen in suspected cat-scratch disease. Lancet, 1992. 339(8807): p. 1443-5.

84. Perkins BA, Swaminathan B, et al., Case 22-1992: Pathogenesis of Cat Scratch Disease. New England Journal of Medicine, 1992. 327(22): p. 1599-601. 
85. Regnery RL, Anderson BE, et al., Characterization of a novel Rochalimaea species, $R$. henselae sp. nov., isolated from blood of a febrile, human immunodeficiency viruspositive patient. J Clin Microbiol, 1992. 30(2): p. 265-74.

86. Welch DF, Pickett DA, et al., Rochalimaea henselae sp. nov., a cause of septicemia, bacillary angiomatosis, and parenchymal bacillary peliosis. Journal of Clinical Microbiology, 1992. 30(2): p. 275-80.

87. Brenner DJ, O'connor SP, et al., Proposals to unify the genera Bartonella and Rochalimaea, with descriptions of Bartonella quintana comb. nov., Bartonella vinsonii comb. nov., Bartonella henselae comb. nov., and Bartonella elizabethae comb. nov., and to remove the family Bartonellaceae from the order Rickettsiales. Int J Syst Bacteriol, 1993. 43(4): p. 777-86.

88. Fediaf the European Pet Food Industry Federation, FEDIAF Facts and Figures 2014. 2014.

89. Cfhs Canadian Federation of Humane Societies, Cats in Canada - A Comprehensive Report on the Cat Overpopulation Crisis. 2012.

90. Appa American Pet Products Association, 2015-2016 APPA National Pet Owners Survey. 2016.

91. Nzcac New Zealand Companion Animal Council, Companion Animals in New Zealand. 2011.

92. Arvand M, Klose AJ, et al., Genetic variability and prevalence of Bartonella henselae in cats in Berlin, Germany, and analysis of its genetic relatedness to a strain from Berlin that is pathogenic for humans. J Clin Microbiol, 2001. 39(2): p. 743-6.

93. Chomel BB, Abbott RC, et al., Bartonella henselae prevalence in domestic cats in California: risk factors and association between bacteremia and antibody titers. Journal of Clinical Microbiology, 1995. 33(9): p. 2445-50.

94. Jameson P, Greene $\mathbf{C}$, et al., Prevalence of Bartonella henselae antibodies in pet cats throughout regions of North America. J Infect Dis, 1995. 172(4): p. 1145-9.

95. Bond R, Riddle A, et al., Survey of flea infestation in dogs and cats in the United Kingdom during 2005. Vet Rec, 2007. 160(15): p. 503-6.

96. Cantó GJ, Guerrero RI, et al., Prevalence of Fleas and Gastrointestinal Parasites in FreeRoaming Cats in Central Mexico. PLoS One, 2013. 8(4): p. e60744.

97. Šlapeta J, King J, et al., The cat flea (Ctenocephalides $f$. felis) is the dominant flea on domestic dogs and cats in Australian veterinary practices. Vet Parasitol, 2011. 180(34): p. 383-88.

98. Harman DW, Halliwell RE, et al., Flea species from dogs and cats in north-central Florida. Vet Parasitol, 1987. 23(1-2): p. 135-40.

99. Koutinas AF, Papazahariadou MG, et al., Flea species from dogs and cats in northern Greece: environmental and clinical implications. Vet Parasitol, 1995. 58(1-2): p. 10915.

100. Silverman J, Rust MK, et al., Influence of temperature and humidity on survival and development of the cat flea, Ctenocephalides felis (Siphonaptera: Pulicidae). J Med Entomol, 1981. 18(1): p. 78-83.

101. Koehler JE, Glaser CA, et al., Rochalimaea henselae infection. A new zoonosis with the domestic cat as reservoir. JAMA, 1994. 271(7): p. 531-5.

102. Higgins JA, Radulovic S, et al., Acquisition of the Cat Scratch Disease Agent Bartonella henselae by Cat Fleas (Siphonaptera: Pulicidae). J Med Entomol, 1996. 33(3): p. 49095.

103. Chomel BB, Kasten RW, et al., Experimental transmission of Bartonella henselae by the cat flea. J Clin Microbiol, 1996. 34(8): p. 1952-6. 
104. Morick D, Krasnov BR, et al., Investigation of Bartonella acquisition and transmission in Xenopsylla ramesis fleas (Siphonaptera: Pulicidae). Mol Ecol, 2011. 20(13): p. 286470.

105. Finkelstein JL, Brown TP, et al., Studies on the growth of Bartonella henselae in the cat flea (Siphonaptera: Pulicidae). J Med Entomol, 2002. 39(6): p. 915-9.

106. Bruce WN, Studies on the Biological Requirements of the Cat Flea. Annals of the Entomological Society of America, 1948. 41(3): p. 346-52.

107. Morick D, Krasnov BR, et al., Vertical nontransovarial transmission of Bartonella in fleas. Mol Ecol, 2013. 22(18): p. 4747-52.

108. Bouhsira E, Franc M, et al., Assessment of persistence of Bartonella henselae in Ctenocephalides felis. Appl Environ Microbiol, 2013. 79(23): p. 7439-44.

109. Dryden MW, Host association, on-host longevity and egg production of Ctenocephalides felis felis. Vet Parasitol, 1989. 34(1): p. 117-22.

110. Dyachenko $\mathbf{P}$, Ziv $\mathbf{M}$, et al., Cat scratch disease encephalopathy in an immunocompetent patient. European Journal of Internal Medicine, 2005. 16(8): p. 610-11.

111. Zangwill KM, Hamilton DH, et al., Cat Scratch Disease in Connecticut -- Epidemiology, Risk Factors, and Evaluation of a New Diagnostic Test. New England Journal of Medicine, 1993. 329(1): p. 8-13.

112. Sanguinetti-Morelli D, Angelakis E, et al., Seasonality of cat-scratch disease, France, 1999-2009. Emerging Infectious Diseases, 2011. 17: p. 705+.

113. Breitschwerdt EB, Maggi RG, et al., Bartonella sp. Bacteremia in Patients with Neurological and Neurocognitive Dysfunction. Journal of Clinical Microbiology, 2008. 46(9): p. 2856-61.

114. Margileth AM, Wear DJ, et al., Systemic Cat Scratch Disease: Report of 23 Patients with Prolonged or Recurrent Severe Bacterial Infection. Journal of Infectious Diseases, 1987. 155(3): p. 390-402.

115. Jacobs RF and Schutze GE, Bartonella henselae as a cause of prolonged fever and fever of unknown origin in children. Clinical Infectious Diseases, 1998. 26(1): p. 80-84.

116. Giladi M, Maman E, et al., Cat-scratch disease-associated arthropathy. Arthritis Rheum, 2005. 52(11): p. 3611-7.

117. Arvand M and Schäd SG, Isolation of Bartonella henselae DNA from the Peripheral Blood of a Patient with Cat Scratch Disease up to 4 Months after the Cat Scratch Injury. Journal of Clinical Microbiology, 2006. 44(6): p. 2288-90.

118. Florin TA, Zaoutis TE, et al., Beyond cat scratch disease: widening spectrum of Bartonella henselae infection. Pediatrics, 2008. 121(5): p. e1413-25.

119. Lucey D, Dolan MJ, et al., Relapsing illness due to Rochalimaea henselae in immunocompetent hosts: implication for therapy and new epidemiological associations. Clin Infect Dis, 1992. 14(3): p. 683-8.

120. Abino JF, Peraldi R, et al., Bacillary splenitis (Bartonella henselae) during immune restoration in an HIV-infected patient. Aids, 2002. 16(10): p. 1429-30.

121. Liston TE and Koehler JE, Granulomatous hepatitis and necrotizing splenitis due to Bartonella henselae in a patient with cancer: case report and review of hepatosplenic manifestations of bartonella infection. Clin Infect Dis, 1996. 22(6): p. 951-7.

122. Salgado CD and Weisse ME, Transverse myelitis associated with probable cat-scratch disease in a previously healthy pediatric patient. Clin Infect Dis, 2000. 31(2): p. 609-11.

123. Awad A and Stüve O, Idiopathic Transverse Myelitis and Neuromyelitis Optica: Clinical Profiles, Pathophysiology and Therapeutic Choices. Current Neuropharmacology, 2011. 9(3): p. 417-28.

124. Brazis PW, Stokes HR, et al., Optic neuritis in cat scratch disease. J Clin Neuroophthalmol, 1986. 6(3): p. 172-4. 
125. Buzzacco DM, Lubow M, et al., Atypical cat scratch disease with vitritis, serous macular detachment, neuroretinitis, and retrobulbar optic neuritis. Albrecht von Graefes Archiv fur klinische und experimentelle Ophthalmologie, 2013. 251(3): p. 10.1007/s00417-012-1967.

126. Kalogeropoulos C, Koumpoulis I, et al., Bartonella and intraocular inflammation: a series of cases and review of literature. Clinical Ophthalmology (Auckland, NZ), 2011. 5: p. 817-29.

127. Selby G and Walker GL, Cerebral arteritis in cat-scratch disease. Neurology, 1979. 29(10): p. 1413-8.

128. Gray AV, Reed JB, et al., Bartonella henselae infection associated with peripapillary angioma, branch retinal artery occlusion, and severe vision loss. Am J Ophthalmol, 1999. 127(2): p. 223-4.

129. Cherinet $\mathbf{Y}$ and Tomlinson $\mathbf{R}$, Cat scratch disease presenting as acute encephalopathy. Emerg Med J, 2008. 25(10): p. 703-4.

130. Carithers HA and Margileth AM, Cat-scratch disease. Acute encephalopathy and other neurologic manifestations. Am J Dis Child, 1991. 145(1): p. 98-101.

131. Rocha JL, Pellegrino LN, et al., Acute hemiplegia associated with cat-scratch disease. Brazilian Journal of Infectious Diseases, 2004. 8: p. 263-66.

132. Spach DH, Panther LA, et al., Intracerebral Bacillary Angiomatosis in a Patient Infected with Human Immunodeficiency Virus. Annals of Internal Medicine, 1992. 116(9): p. 740-42.

133. Schwartzman WA, Patnaik M, et al., Rochalimaea antibodies in HIV-associated neurologic disease. Neurology, 1994. 44(7): p. 1312-6.

134. Schwartzman WA, Patnaik M, et al., Bartonella (Rochalimaea) antibodies, dementia, and cat ownership among men infected with human immunodeficiency virus. Clin Infect Dis, 1995. 21(4): p. 954-9.

135. Metzkor-Cotter E, Kletter Y, et al., Long-term serological analysis and clinical followup of patients with cat scratch disease. Clin Infect Dis, 2003. 37(9): p. 1149-54.

136. Bergmans AM, Peeters MF, et al., Pitfalls and fallacies of cat scratch disease serology: evaluation of Bartonella henselae-based indirect fluorescence assay and enzymelinked immunoassay. J Clin Microbiol, 1997. 35(8): p. 1931-7.

137. Slater MR, Di Nardo A, et al., Free-roaming dogs and cats in central Italy: public perceptions of the problem. Prev Vet Med, 2008. 84(1-2): p. 27-47.

138. Tasker L, Wspa, et al., Report: Stray Animal Control Practises (Europe) - An Investigation of Stray Dog and Cat Population Control Practises Across Europe. 2007.

139. Joseph AK, Wood CW, et al., Bartonella henselae bacteraemia in domestic cats from Auckland. N Z Vet J, 1997. 45(5): p. 185-7.

140. Kelly P, Rolain JM, et al., Prevalence of human pathogens in cat and dog fleas in New Zealand. N Z Med J, 2005. 118(1226): p. U1754.

141. Schiellerup P, Dyhr T, et al., Low seroprevalence of bartonella species in danish elite orienteers. Scand J Infect Dis, 2004. 36(8): p. 604-6.

142. Mcgill S, Wesslen L, et al., Bartonella spp. seroprevalence in healthy Swedish blood donors. Scand J Infect Dis, 2005. 37(10): p. 723-30.

143. Harrison TG and Doshi N, Serological evidence of Bartonella spp. infection in the UK. Epidemiol Infect, 1999. 123(2): p. 233-40.

144. Kumasaka K, Arashima Y, et al., Survey of veterinary professionals for antibodies to Bartonella henselae in Japan. Rinsho Byori, 2001. 49(9): p. 906-10.

145. Zarkovic A, Macmurray C, et al., Seropositivity rates for Bartonella henselae, Toxocara canis and Toxoplasma gondii in New Zealand blood donors. Clinical \& Experimental Ophthalmology, 2007. 35(2): p. 131-34. 
146. Maruyama S, Boonmar S, et al., Seroprevalence of Bartonella henselae and Toxoplasma gondii among healthy individuals in Thailand. J Vet Med Sci, 2000. 62(6): p. 635-7.

147. Yilmaz C, Ergin C, et al., Investigation of Bartonella henselae seroprevalence and related risk factors in blood donors admitted to Pamukkale University Blood Center. Mikrobiyol Bul, 2009. 43(3): p. 391-401.

148. Pons I, Sanfeliu I, et al., Serological evidence of Bartonella henselae infection in healthy people in Catalonia, Spain. Epidemiol Infect, 2008. 136(12): p. 1712-16.

149. Al-Majali AM and AI-Qudah KM, Seroprevalence of Bartonella henselae and Bartonella quintana infections in children from Central and Northern Jordan. Saudi Med J, 2004. 25(11): p. 1664-9.

150. Sander A, Berner R, et al., Serodiagnosis of cat scratch disease: response to Bartonella henselae in children and a review of diagnostic methods. Eur J Clin Microbiol Infect Dis, 2001. 20(6): p. 392-401.

151. Costa PS, Brigatte ME, et al., Antibodies to Rickettsia rickettsii, Rickettsia typhi, Coxiella burnetii, Bartonella henselae, Bartonella quintana, and Ehrlichia chaffeensis among healthy population in Minas Gerais, Brazil. Memórias do Instituto Oswaldo Cruz, 2005. 100: p. 853-59.

152. Sun J, Fu G, et al., Seroprevalence of Bartonella in Eastern China and analysis of risk factors. BMC Infectious Diseases, 2010. 10(1): p. 1-4.

153. Hong Kong Census and Statistics Department, Thematic Household Survey Report No. 482011.

154. Garcia-Garcia JA, Baquerizo R, et al., Prevalence of serum antibodies against Bartonella ssp. in a health population from the south area of the Seville province. Rev Clin Esp, 2005. 205(11): p. 541-4.

155. Sander A, Posselt M, et al., Seroprevalence of Antibodies to Bartonella henselae in Patients with Cat Scratch Disease and in Healthy Controls: Evaluation and Comparison of Two Commercial Serological Tests. Clinical and Diagnostic Laboratory Immunology, 1998. 5(4): p. 486-90.

156. Lamas CC, Mares-Guia MA, et al., Bartonella spp. infection in HIV positive individuals, their pets and ectoparasites in Rio de Janeiro, Brazil: serological and molecular study. Acta Trop, 2010. 115(1-2): p. 137-41.

157. Cimolai N, Benoit L, et al., Bartonella henselae infection in British Columbia: evidence for an endemic disease among humans. Can J Microbiol, 2000. 46(10): p. 908-12.

158. Pandak N, Dakovic-Rode O, et al., Prevalence of Bartonella henselae antibodies in children and blood donors in Croatia. Infection, 2009. 37(2): p. 166-7.

159. Massei F, Messina F, et al., High Prevalence of Antibodies to Bartonella henselae among Italian Children without Evidence of Cat Scratch Disease. Clinical Infectious Diseases, 2004. 38(1): p. 145-48.

160. Karem, Dubois, et al., Characterization of Bartonella henselae-specific immunity in $B A L B / c$ mice. Immunology, 1999. 97(2): p. 352-58.

161. Okujava R, Guye P, et al., A Translocated Effector Required for Bartonella Dissemination from Derma to Blood Safeguards Migratory Host Cells from Damage by Co-translocated Effectors. PLoS Pathog, 2014. 10(6): p. e1004187.

162. Dehio C, Bartonella-host-cell interactions and vascular tumour formation. Nat Rev Micro, 2005. 3(8): p. 621-31.

163. Mandle $\mathrm{T}$, Einsele $\mathbf{H}$, et al., Infection of human CD34+ progenitor cells with Bartonella henselae results in intraerythrocytic presence of B. henselae. Blood, 2005. 106(4): p. 1215-22. 
164. Salvatore P, Casamassimi A, et al., Detrimental effects of Bartonella henselae are counteracted by L-arginine and nitric oxide in human endothelial progenitor cells. Proc Natl Acad Sci U S A, 2008. 105(27): p. 9427-32.

165. Batterman HJ, Peek JA, et al., Bartonella henselae and Bartonella quintana adherence to and entry into cultured human epithelial cells. Infect Immun, 1995. 63(11): p. 45536.

166. Kempf VA, Schairer A, et al., Bartonella henselae inhibits apoptosis in Mono Mac 6 cells. Cell Microbiol, 2005. 7(1): p. 91-104.

167. Kosoy MY, Regnery RL, et al., Experimental infection of cotton rats with three naturally occurring Bartonella species. J Wildl Dis, 1999. 35(2): p. 275-84.

168. Pulliainen AT and Dehio C, Persistence of Bartonella spp. stealth pathogens: from subclinical infections to vasoproliferative tumor formation. FEMS Microbiol Rev, 2012. 36(3): p. 563-99.

169. Popa C, Abdollahi-Roodsaz S, et al., Bartonella quintana lipopolysaccharide is a natural antagonist of Toll-like receptor 4. Infect Immun, 2007. 75(10): p. 4831-7.

170. Zhang P, Chomel BB, et al., A family of variably expressed outer-membrane proteins (Vomp) mediates adhesion and autoaggregation in Bartonella quintana. Proc Natl Acad Sci U S A, 2004. 101(37): p. 13630-5.

171. Marignac G, Barrat F, et al., Murine model for Bartonella birtlesii infection: New aspects. Comp Immunol Microbiol Infect Dis, 2010. 33(2): p. 95-107.

172. Riess T, Andersson SG, et al., Bartonella adhesin a mediates a proangiogenic host cell response. J Exp Med, 2004. 200(10): p. 1267-78.

173. Rahman MM and Mcfadden G, Modulation of NF-kappaB signalling by microbial pathogens. Nat Rev Microbiol, 2011. 9(4): p. 291-306.

174. Kaiser PO, Riess T, et al., The head of Bartonella adhesin A is crucial for host cell interaction of Bartonella henselae. Cell Microbiol, 2008. 10(11): p. 2223-34.

175. Schmid MC, Schulein R, et al., The VirB type IV secretion system of Bartonella henselae mediates invasion, proinflammatory activation and antiapoptotic protection of endothelial cells. Mol Microbiol, 2004. 52(1): p. 81-92.

176. Vayssier-Taussat M, Le Rhun $\mathbf{D}$, et al., The Trw type IV secretion system of Bartonella mediates host-specific adhesion to erythrocytes. PLoS Pathog, 2010. 6(6): p. e1000946.

177. Rhomberg TA, Truttmann MC, et al., A translocated protein of Bartonella henselae interferes with endocytic uptake of individual bacteria and triggers uptake of large bacterial aggregates via the invasome. Cell Microbiol, 2009. 11(6): p. 927-45.

178. Kyme PA, Haas A, et al., Unusual trafficking pattern of Bartonella henselae -containing vacuoles in macrophages and endothelial cells. Cell Microbiol, 2005. 7(7): p. 1019-34.

179. Etchegaray JP and Inouye $\mathbf{M}, \operatorname{CspA}, \operatorname{CspB}$, and $\operatorname{CspG}$, major cold shock proteins of Escherichia coli, are induced at low temperature under conditions that completely block protein synthesis. J Bacteriol, 1999. 181(6): p. 1827-30.

180. Arsene F, Tomoyasu T, et al., The heat shock response of Escherichia coli. Int J Food Microbiol, 2000. 55(1-3): p. 3-9.

181. Raivio TL, Envelope stress responses and Gram-negative bacterial pathogenesis. Mol Microbiol, 2005. 56(5): p. 1119-28.

182. Potrykus $\mathrm{K}$, Murphy $\mathbf{H}$, et al., ppGpp is the major source of growth rate control in $E$. coli. Environ Microbiol, 2011. 13(3): p. 563-75.

183. Kazmierczak MJ, Mithoe SC, et al., Listeria monocytogenes sigma B regulates stress response and virulence functions. J Bacteriol, 2003. 185(19): p. 5722-34.

184. Schreiber G, Ron EZ, et al., ppGpp-mediated regulation of DNA replication and cell division in Escherichia coli. Curr Microbiol, 1995. 30(1): p. 27-32.

185. Pittman RJ, Kline CLK, et al., Carbon-Starvation Induces Cross-Resistance to Thermal, Acid, and Oxidative Stress in Serratia marcescens. Microorganisms, 2015. 3(4). 
186. Tu N, Lima A, et al., Characterization of the general stress response in Bartonella henselae. Microb Pathog, 2016. 92: p. 1-10.

187. Atkinson GC, Tenson T, et al., The RelA/SpoT homolog (RSH) superfamily: distribution and functional evolution of ppGpp synthetases and hydrolases across the tree of life. PLoS One, 2011. 6(8): p. e23479.

188. Ferullo DJ and Lovett ST, The stringent response and cell cycle arrest in Escherichia coli. PLoS Genet, 2008. 4(12): p. e1000300.

189. Durfee T, Hansen AM, et al., Transcription profiling of the stringent response in Escherichia coli. J Bacteriol, 2008. 190(3): p. 1084-96.

190. Maisonneuve E, Castro-Camargo M, et al., (p)ppGpp Controls Bacterial Persistence by Stochastic Induction of Toxin-Antitoxin Activity. Cell, 2013. 154(5): p. 1140-50.

191. Bittner AN, Kriel A, et al., Lowering GTP level increases survival of amino acid starvation but slows growth rate for Bacillus subtilis cells lacking (p)ppGpp. J Bacteriol, 2014. 196(11): p. 2067-76.

192. Dalebroux ZD, Svensson SL, et al., ppGpp Conjures Bacterial Virulence. Microbiology and Molecular Biology Reviews : MMBR, 2010. 74(2): p. 171-99.

193. Mackow ER and Chang FN, Correlation between RNA synthesis and ppGpp content in Escherichia coli during temperature shifts. Mol Gen Genet, 1983. 192(1-2): p. 5-9.

194. Nguyen D, Joshi-Datar A, et al., Active starvation responses mediate antibiotic tolerance in biofilms and nutrient-limited bacteria. Science (New York, NY), 2011. 334(6058): p. 982-86.

195. Khakimova M, Ahlgren HG, et al., The Stringent Response Controls Catalases in Pseudomonas aeruginosa and Is Required for Hydrogen Peroxide and Antibiotic Tolerance. Journal of Bacteriology, 2013. 195(9): p. 2011-20.

196. Jenkins DE, Schultz JE, et al., Starvation-induced cross protection against heat or H2O2 challenge in Escherichia coli. Journal of Bacteriology, 1988. 170(9): p. 3910-14.

197. Imlay JA, Cellular defenses against superoxide and hydrogen peroxide. Annual review of biochemistry, 2008. 77: p. 755-76.

198. Cashel M and Gallant J, Two compounds implicated in the function of the RC gene of Escherichia coli. Nature, 1969. 221(5183): p. 838-41.

199. Cashel M and Kalbacher B, The control of ribonucleic acid synthesis in Escherichia coli. $V$. Characterization of a nucleotide associated with the stringent response. J Biol Chem, 1970. 245(9): p. 2309-18.

200. Zuo Y, Wang Y, et al., The Mechanism of E. coli RNA Polymerase Regulation by ppGpp Is Suggested by the Structure of Their Complex. Molecular cell, 2013. 50(3): p. 430-36.

201. Jishage $\mathbf{M}$, Kvint $\mathrm{K}$, et al., Regulation of sigma factor competition by the alarmone ppGpp. Genes Dev, 2002. 16(10): p. 1260-70.

202. Osterberg S, Del Peso-Santos T, et al., Regulation of alternative sigma factor use. Annu Rev Microbiol, 2011. 65: p. 37-55.

203. Gopalkrishnan S, Nicoloff $\mathbf{H}$, et al., Co-ordinated regulation of the extracytoplasmic stress factor, sigmaE, with other Escherichia coli sigma factors by (p)ppGpp and DksA may be achieved by specific regulation of individual holoenzymes. Mol Microbiol, 2014. 93(3): p. 479-93.

204. Gaca AO, Kajfasz JK, et al., Basal levels of (p)ppGpp in Enterococcus faecalis: the magic beyond the stringent response. MBio, 2013. 4(5): p. e00646-13.

205. Kriel A, Bittner AN, et al., Direct regulation of GTP homeostasis by (p)ppGpp: a critical component of viability and stress resistance. Mol Cell, 2012. 48(2): p. 231-41.

206. Potrykus K and Cashel M, (p)ppGpp: still magical? Annu Rev Microbiol, 2008. 62: p. 35-51. 
207. Quebatte M, Dick MS, et al., Dual input control: activation of the Bartonella henselae VirB/D4 type IV secretion system by the stringent sigma factor RpoH1 and the BatR/BatS two-component system. Mol Microbiol, 2013. 90(4): p. 756-75.

208. Quebatte $\mathbf{M}$, Dehio $\mathbf{M}$, et al., The BatR/BatS two-component regulatory system controls the adaptive response of Bartonella henselae during human endothelial cell infection. J Bacteriol, 2010. 192(13): p. 3352-67.

209. Perederina A, Svetlov V, et al., Regulation through the Secondary Channel-Structural Framework for ppGpp-DksA Synergism during Transcription. Cell, 2004. 118(3): p. 297309.

210. Paul BJ, Berkmen MB, et al., DksA potentiates direct activation of amino acid promoters by ppGpp. Proc Natl Acad Sci U S A, 2005. 102(22): p. 7823-8.

211. Paul BJ, Barker MM, et al., DksA: a critical component of the transcription initiation machinery that potentiates the regulation of rRNA promoters by ppGpp and the initiating NTP. Cell, 2004. 118(3): p. 311-22.

212. Stallings CL, Stephanou NC, et al., CarD is an essential regulator of rRNA transcription required for Mycobacterium tuberculosis persistence. Cell, 2009. 138(1): p. 146-59.

213. Elías-Arnanz $\mathbf{M}$, Fontes $\mathbf{M}$, et al., 12 Carotenogenesis in Myxococcus xanthus: a Complex Regulatory Network, in Myxobacteria. 2008, American Society of Microbiology.

214. Galbis-Martinez M, Fontes M, et al., The high-mobility group A-type protein CarD of the bacterium Myxococcus xanthus as a transcription factor for several distinct vegetative genes. Genetics, 2004. 167(4): p. 1585-95.

215. Gallego-García A, Mirassou Y, et al., Structural Insights into RNA Polymerase Recognition and Essential Function of Myxococcus xanthus CdnL. PLoS One, 2014. 9(10): p. e108946.

216. Srivastava DB, Leon $\mathrm{K}$, et al., Structure and function of CarD, an essential mycobacterial transcription factor. Proc Natl Acad Sci U S A, 2013. 110(31): p. 1261924.

217. Kaur G, Dutta D, et al., Crystal structure of Mycobacterium tuberculosis CarD, an essential RNA polymerase binding protein, reveals a quasidomain-swapped dimeric structural architecture. Proteins, 2014. 82(5): p. 879-84.

218. Rammohan J, Ruiz manzano A, et al., CarD stabilizes mycobacterial open complexes via a two-tiered kinetic mechanism. Nucleic Acids Res, 2015. 43(6): p. 3272-85.

219. Gulten G and Sacchettini JC, Structure of the Mtb CarD/RNAP beta-lobes complex reveals the molecular basis of interaction and presents a distinct DNA-binding domain for Mtb CarD. Structure, 2013. 21(10): p. 1859-69.

220. Bae B, Chen J, et al., CarD uses a minor groove wedge mechanism to stabilize the RNA polymerase open promoter complex. Elife, 2015. 4.

221. Davis E, Chen J, et al., Mycobacterial RNA polymerase forms unstable open promoter complexes that are stabilized by CarD. Nucleic Acids Res, 2015. 43(1): p. 433-45.

222. Stallings $\mathrm{CL}$ and Glickman MS, CarD: a new RNA polymerase modulator in mycobacteria. Transcription, 2011. 2(1): p. 15-8.

223. Weiss LA, Harrison PG, et al., Interaction of CarD with RNA Polymerase Mediates Mycobacterium tuberculosis Viability, Rifampin Resistance, and Pathogenesis. Journal of Bacteriology, 2012. 194(20): p. 5621-31.

224. Yang XF, Goldberg MS, et al., Differential expression of a putative CarD-like transcriptional regulator, LtpA, in Borrelia burgdorferi. Infect Immun, 2008. 76(10): p. 4439-44.

225. Garcia-Moreno D, Polanco MC, et al., A vitamin B12-based system for conditional expression reveals dksA to be an essential gene in Myxococcus xanthus. J Bacteriol, 2009. 191(9): p. 3108-19. 
226. Hoang TT, Karkhoff-Schweizer RR, et al., A broad-host-range Flp-FRT recombination system for site-specific excision of chromosomally-located DNA sequences: application for isolation of unmarked Pseudomonas aeruginosa mutants. Gene, 1998. 212(1): p. 77-86.

227. Schulein R and Dehio C, The VirB/VirD4 type IV secretion system of Bartonella is essential for establishing intraerythrocytic infection. Mol Microbiol, 2002. 46(4): p. 1053-67.

228. Mackichan JK, Gerns $\mathrm{HL}$, et al., A SacB mutagenesis strategy reveals that the Bartonella quintana variably expressed outer membrane proteins are required for bloodstream infection of the host. Infect Immun, 2008. 76(2): p. 788-95.

229. Atlas RM, Handbook of media for clinical microbiology Boca Raton; CRC Press, 2006, 2006.

230. Roden JA, Wells DH, et al., Hemin Binding Protein C Is Found in Outer Membrane Vesicles and Protects Bartonella henselae against Toxic Concentrations of Hemin. Infect Immun, 2012. 80(3): p. 929-42.

231. Rieß T, Anderson B, et al., Rapid and efficient transposon mutagenesis of Bartonella henselae by transposome technology. Gene, 2003. 313: p. 103-09.

232. Wilson K, Preparation of Genomic DNA from Bacteria, in Current Protocols in Molecular Biology. 2001, John Wiley \& Sons, Inc.

233. Kearse M, Moir R, et al., Geneious Basic: An integrated and extendable desktop software platform for the organization and analysis of sequence data. Bioinformatics, 2012. 28(12): p. 1647-49.

234. Goujon $\mathbf{M}, \mathbf{M c w i l l i a m} \mathbf{H}$, et al., A new bioinformatics analysis tools framework at EMBL-EBI. Nucleic Acids Res, 2010. 38: p. W695-9.

235. Resto-Ruiz SI, Sweger D, et al., Transcriptional activation of the htrA (Hightemperature requirement A) gene from Bartonella henselae. Infect Immun, 2000. 68(10): p. 5970-8.

236. Riess T, Anderson B, et al., Rapid and efficient transposon mutagenesis of Bartonella henselae by transposome technology. Gene, 2003. 313: p. 103-09.

237. Geissmann Q, OpenCFU, a New Free and Open-Source Software to Count Cell Colonies and Other Circular Objects. PLoS One, 2013. 8(2): p. e54072.

238. Gay $\mathbf{P}$, Le Coq $\mathbf{D}$, et al., Positive selection procedure for entrapment of insertion sequence elements in gram-negative bacteria. Journal of Bacteriology, 1985. 164(2): p. 918-21.

239. Smillie C, Garcillan-Barcia MP, et al., Mobility of plasmids. Microbiol Mol Biol Rev, 2010. 74(3): p. 434-52.

240. Lima A, Cha BJ, et al., Zebrafish Embryo Model of Bartonella henselae Infection. Zebrafish, 2014. 11(5): p. 434-46.

241. Simon R, Priefer U, et al., A Broad Host Range Mobilization System for In Vivo Genetic Engineering: Transposon Mutagenesis in Gram Negative Bacteria. Nat Biotech, 1983. 1(9): p. 784-91.

242. Strand TA, Lale R, et al., A New and Improved Host-Independent Plasmid System for RK2-Based Conjugal Transfer. PLoS One, 2014. 9(3): p. e90372.

243. Dehio $C$ and Meyer $M$, Maintenance of broad-host-range incompatibility group $P$ and group Q plasmids and transposition of Tn5 in Bartonella henselae following conjugal plasmid transfer from Escherichia coli. Journal of Bacteriology, 1997. 179(2): p. 53840.

244. Grasseschi HA and Minnick MF, Transformation of Bartonella bacilliformis by electroporation. Can J Microbiol, 1994. 40(9): p. 782-6. 
245. Garcia-Moreno D, Abellon-Ruiz J, et al., CdnL, a member of the large CarD-like family of bacterial proteins, is vital for Myxococcus xanthus and differs functionally from the global transcriptional regulator CarD. Nucleic Acids Res, 2010. 38(14): p. 4586-98.

246. Abromaitis S, Nelson CS, et al., Bartonella quintana deploys host and vector temperature-specific transcriptomes. PLoS One, 2013. 8(3): p. e58773.

247. Sander A, Kretzer S, et al., Hemin-dependent growth and hemin binding of Bartonella henselae. FEMS Microbiology Letters, 2000. 189(1): p. 55-59.

248. Carroll JA, Coleman SA, et al., Hemin-binding surface protein from Bartonella quintana. Infect Immun, 2000. 68(12): p. 6750-7.

249. Myers WF, Osterman JV, et al., Nutritional studies of Rickettsia guintana: nature of the hematin requirement. J Bacteriol, 1972. 109(1): p. 89-95.

250. Parrow NL, Abbott J, et al., Function, Regulation, and Transcriptional Organization of the Hemin Utilization Locus of Bartonella quintana. Infect Immun, 2009. 77(1): p. 30716.

251. Schmitt TH, Frezzatti WA, et al., Hemin-Induced Lipid Membrane Disorder and Increased Permeability: A Molecular Model for the Mechanism of Cell Lysis. Archives of Biochemistry and Biophysics, 1993. 307(1): p. 96-103.

252. Graça-Souza AV, Maya-Monteiro C, et al., Adaptations against heme toxicity in bloodfeeding arthropods. Insect Biochemistry and Molecular Biology, 2006. 36(4): p. 32235.

253. Liu M, Boulouis H-J, et al., Heme Degrading Protein HemS Is Involved in Oxidative Stress Response of Bartonella henselae. PLoS One, 2012. 7(5): p. e37630.

254. Liu M, Ferrandez Y, et al., Heme Binding Proteins of Bartonella henselae Are Required when Undergoing Oxidative Stress During Cell and Flea Invasion. PLoS One, 2012. 7(10): p. e48408.

255. Battisti JM, Sappington KN, et al., Environmental Signals Generate a Differential and Coordinated Expression of the Heme Receptor Gene Family of Bartonella quintana. Infect Immun, 2006. 74(6): p. 3251-61.

256. Pendle S, Ginn A, et al., Antimicrobial susceptibility of Bartonella henselae using Etest methodology. Journal of Antimicrobial Chemotherapy, 2006. 57(4): p. 761-63.

257. Drlica K, Mechanism of fluoroquinolone action. Current Opinion in Microbiology, 1999. 2(5): p. 504-08.

258. Petersen K, Earhart KC, et al., Bacillary Angiomatosis in a Patient with Chronic Lymphocytic Leukemia. Infection, 2008. 36(5): p. 480-84.

259. Tsuneoka H, Yanagihara M, et al., Antimicrobial susceptibility by Etest of Bartonella henselae isolated from cats and human in Japan. Journal of Infection and Chemotherapy. 16(6): p. 446-48.

260. Lushchak VI, Oxidative stress and mechanisms of protection against it in bacteria. Biochemistry (Mosc), 2001. 66(5): p. 476-89.

261. Dryden M, Biology of the Cat Flea, Ctenocephalides felis felis. Companion animal practice (USA), 1989.

262. Hinkle NC, Koehler PG, et al., Hematophagous Strategies of the Cat Flea (Siphonaptera: Pulicidae). The Florida Entomologist, 1991. 74(3): p. 377-85.

263. Drecktrah D, Lybecker M, et al., The Borrelia burgdorferi RelA/SpoT Homolog and Stringent Response Regulate Survival in the Tick Vector and Global Gene Expression during Starvation. PLoS Pathog, 2015. 11(9): p. e1005160.

264. Abromaitis S and Koehler JE, The Bartonella quintana extracytoplasmic function sigma factor RpoE has a role in bacterial adaptation to the arthropod vector environment. J Bacteriol, 2013. 195(11): p. 2662-74. 
265. Stauff DL, Bagaley D, et al., Staphylococcus aureus HrtA is an ATPase required for protection against heme toxicity and prevention of a transcriptional heme stress response. J Bacteriol, 2008. 190(10): p. 3588-96.

266. Liu X, Olczak T, et al., Identification of Amino Acid Residues Involved in Heme Binding and Hemoprotein Utilization in the Porphyromonas gingivalis Heme Receptor HmuR. Infect Immun, 2006. 74(2): p. 1222-32.

267. Zimmermann R, Kempf VAJ, et al., Hemin Binding, Functional Expression, and Complementation Analysis of Pap 31 from Bartonella henselae. Journal of Bacteriology, 2003. 185(5): p. 1739-44.

268. Speare R, Canyon DV, et al., Quantification of blood intake of the head louse: Pediculus humanus capitis. Int J Dermatol, 2006. 45(5): p. 543-6.

269. Rust MK and Dryden MW, The biology, ecology, and management of the cat flea. Annu Rev Entomol, 1997. 42: p. 451-73.

270. Müller A, Reiter M, et al., Development of a serum-free liquid medium for Bartonella species. Folia Microbiologica, 2016. 61: p. 393-98.

271. Angelakis E, Biswas S, et al., Heterogeneity of susceptibility to fluoroquinolones in Bartonella isolates from Australia reveals a natural mutation in gyrA. The Journal of antimicrobial chemotherapy, 2008. 61(6): p. 1252-5.

272. Biswas S, Raoult D, et al., Molecular mechanisms of resistance to antibiotics in Bartonella bacilliformis. The Journal of antimicrobial chemotherapy, 2007. 59(6): p. 1065-70.

273. Heisig $\mathbf{P}$ and Tschorny $\mathbf{R}$, Characterization of fluoroquinolone-resistant mutants of escherichia coli selected in vitro. Antimicrob Agents Chemother, 1994. 38(6): p. 128491.

274. Kocagoz T, Hackbarth $\mathrm{CJ}$, et al., Gyrase mutations in laboratory-selected, fluoroquinolone-resistant mutants of Mycobacterium tuberculosis H37Ra. Antimicrob Agents Chemother, 1996. 40(8): p. 1768-74.

275. Minnick MF, Wilson ZR, et al., gyrA mutations in ciprofloxacin-resistant Bartonella bacilliformis strains obtained in vitro. Antimicrob Agents Chemother, 2003. 47(1): p. 383-6.

276. Labombardi VJ and Cataldo-Caputzal L, Ciprofloxacin susceptibility testing by MIC and disk elution of drug-resistant Mycobacterium tuberculosis and Mycobacterium avium complex. Antimicrobial Agents and Chemotherapy, 1993. 37(7): p. 1556-57.

277. Musso T, Badolato R, et al., Interaction of Bartonella henselae with the Murine Macrophage Cell Line J774: Infection and Proinflammatory Response. Infect Immun, 2001. 69(10): p. 5974-80.

278. Coleman SA and Minnick MF, Differential expression of the invasion-associated locus $B$ (ialB) gene of Bartonella bacilliformis in response to environmental cues. Microbial Pathogenesis, 2003. 34(4): p. 179-86.

279. Hanna N, Ouahrani-Bettache S, et al., Global Rsh-dependent transcription profile of Brucella suisduring stringent response unravels adaptation to nutrient starvation and cross-talk with other stress responses. BMC Genomics, 2013. 14(1): p. 1-16.

280. Dumas JL, Van Delden C, et al., Analysis of antibiotic resistance gene expression in Pseudomonas aeruginosa by quantitative real-time-PCR. FEMS Microbiol Lett, 2006. 254(2): p. 217-25.

281. Jiang S, Cieslewicz MJ, et al., Regulation of Virulence by a Two-Component System in Group B Streptococcus. Journal of Bacteriology, 2005. 187(3): p. 1105-13.

282. Dehio $\mathbf{M}$, Quebatte $\mathbf{M}$, et al., The transcriptional response of human endothelial cells to infection with Bartonella henselae is dominated by genes controlling innate immune responses, cell cycle, and vascular remodelling. Thromb Haemost, 2005. 94(2): p. 34761. 
283. Bernal-Bernal D, Gallego-Garcia A, et al., Structure-function dissection of Myxococcus xanthus CarD N-terminal domain, a defining member of the CarD_CdnL_TRCF family of RNA polymerase interacting proteins. PLoS One, 2015. 10(3): p. e0121322.

284. Schneider I, Differentiation of larval drosophila eye-antennal Discs in Vitro. Exp Zool, 1964. 156(1): p. 91.

285. Kakuta K, Orino K, et al., High levels of ferritin and its iron in fetal bovine serum. Comp Biochem Physiol A Physiol, 1997. 118(1): p. 165-9.

286. Seaver LC and Imlay JA, Hydrogen peroxide fluxes and compartmentalization inside growing Escherichia coli. Journal of Bacteriology, 2001. 183(24): p. 7182-89.

287. Liu X, Ramsey MM, et al., Real-time mapping of a hydrogen peroxide concentration profile across a polymicrobial bacterial biofilm using scanning electrochemical microscopy. Proc Natl Acad Sci U S A, 2011. 108(7): p. 2668-73.

288. Trautinger BW, Jaktaji RP, et al., RNA polymerase modulators and DNA repair activities resolve conflicts between DNA replication and transcription. Mol Cell, 2005. 19(2): p. 247-58.

289. Gober JW and Marques MV, Regulation of cellular differentiation in Caulobacter crescentus. Microbiological Reviews, 1995. 59(1): p. 31-47.

290. Curtis PD and Brun YV, Getting in the loop: regulation of development in Caulobacter crescentus. Microbiol Mol Biol Rev, 2010. 74(1): p. 13-41. 


\section{Chapter 8. Appendices}

\subsection{CarD Amino Acid Sequence Alignments}

Alignments were performed using Clustal Omega, a multiple sequence alignment $\operatorname{program}^{[234]}$.

CarD family transcriptional regulator (Bartonella henselae)

NCBI Reference Sequence: WP_011181290.1

CarD family transcriptional regulator (Mycobacterium tuberculosis)

NCBI Reference Sequence: WP_057133327.1

CdnL, CarD family transcriptional regulator (Myxococcus xanthus)

NCBI Reference Sequence: 2LWJ_A

pJS01 CarD is a hypothetical truncated CarD protein encoded by our pJS01 plasmid. This was obtained by analysing the Macrogen sequencing results.

$$
\begin{array}{ll}
\text { B. henselae } & 8 \\
\text { M. tuberculosis } & 3 \\
\text { M. xanthus } & 14 \\
& \\
\text { B. henselae } \\
\text { M. tuberculosis } \\
\text { M. xanthus }
\end{array}
$$

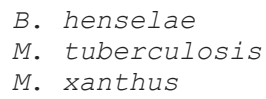

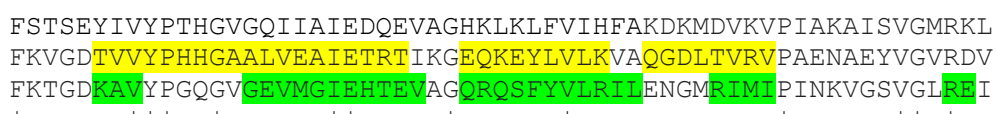

SAVDSVERALKILHGKARV-KRTMWSRRAQEYDAKINSGDLICIAEVVRDLFRSDLQPEQ VGQEGLDKVFQVLRAPHTE-EPTNWSRRYKANLEKLASGDVNKVAEVVRDLWRRDQERGL ISEEDVKQVYS ILKEKDISVDSTTWNRRYREYMEKIKTGSVFEIAEVLRDLYLLKGDKDL

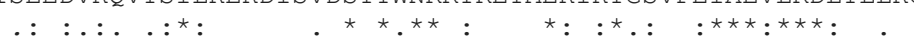

SYSERQLYIAALERMAREIAVINSLSETEAINLIEMHLS $\quad 170$ SAGEKRMLAKARQILVGELALAESTDDAKAETILDEVLAAA 161 SFGERKMLDTARSLLIKELSLAKDCSEDEIESDLKKIFNLA 167

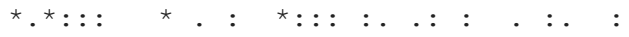

Appendix Figure 8.1. - ClustalOmega Amino Acid Sequence Alignments All sequences were sourced from the $\mathrm{NCBI}$ online gene database and then aligned with Clustal Omega with the exception of pJS01 CarD, which was not included in the initial sequence alignment. The pJS01 truncated CarD sequence shown above is a hypothetical expressed protein segment as determined from our Macrogen sequencing data. $M$. tuberculosis sequence highlighted in yellow denote $\beta 1, \beta 2$, and $\beta 3$ sheets and $M$. xanthus sequence highlighted in green denote $\beta 1, \beta 2, \beta 3, \beta 4$, and $\beta 5$ sheets $[215,217]$. The CarD/CdnL binding to RNA polymerase is through the $\mathrm{N}$-terminal Tudor-like folds of $\beta$ sheets. Though the pJS01 hypothetical CarD protein is heavily truncated, it still includes the $M$. tuberculosis $\beta 1$ and $\beta 2$ folds (a possible truncated $\beta 3$ fold) and $M$. xanthus $\beta 1, \beta 2$, and $\beta 3$ folds (with a possible truncated $\beta 4$ fold). 


\section{2. $\quad$ carD PCR Fusion Reaction Figure}

1)

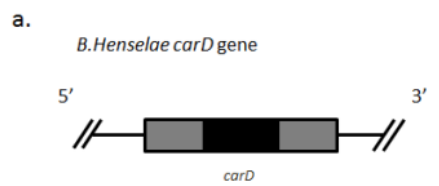

b.

PCR - carD 3' flanking region

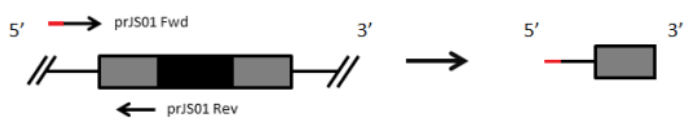

PCR - CarD 5' flanking region

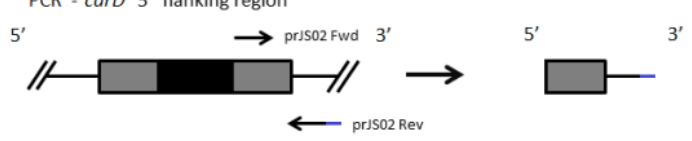

c.

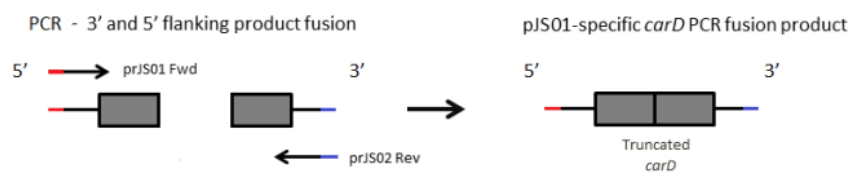

2)

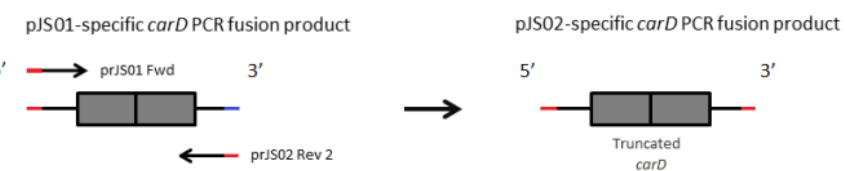

Appendix Figure 8.2. - Synthesis of the carD PCR fusion products for pJS01 and pJS02 1) (a.) Representation of the $B$. henselae carD gene on purified genomic DNA. The segment of $\operatorname{car} D$ coloured in black represents the section that will be removed to form a truncated carD gene, i.e. the pJS01 carD fusion product. (b.) Concurrent PCRs using $B$. henselae gDNA as a template with prJS01 and prJS02 primer pairs to yield the carD $3^{\prime}$ and 5' flanking region products. Arrows indicate direction of primer extension; primer sections coloured red and blue indicate overhangs encoding BamHI and Sall restriction sites respectively. (c.) Fusion PCR using carD flanking region products as template DNA to yield the pJS01 carD PCR fusion product insert. The forward prJS01 and reverse prJS02 primers were used. 2) (a.) The pJS01 carD PCR fusion product was used as a DNA template for a PCR to yield the pJS02 carD PCR fusion product. The forward prJSO1 and reverse 2 prJS02 primers were used. As before, primer sections coloured red indicate overhangs encoding BamHI. 


\subsection{B. henselae carD Gene Sequence}

TTGCGTTGAAGTTTGCCCTGTTGATTGTTTTTATGAAGGTGAAAACATGCTTGTCATTCATCCCGATGAATGTATCGATTGTGGTGT GTGTGAACCAGAATGCCCTGCAGAAGCCATTAAACCGGATACAGAGCCGGGGCTAGAAAAATGGCTAGAGCTCAATCTTCAATATGC AAACAAATGGCCTAATTTAACCACCAGAAAAGATCCTCTTCCTCAAGCAAAAGAAATGGATGGCATTCCGGATAAATTAGAAAAGTA TTTTTCAGAAAATCCAGGAAGCGGAGAATAATAAGCATAGGACATACATCATAAGCTTTTAAAAATTGAAATCATTTCTTTGTAAAA ATAAAAATTCCTCAGTGAAATGATTGATTCTTTTGACATTTGATGTTAATGTTATTTTAACATGATCATCTTTCTAGGTATTTTTCT TGCTTTCTCCTTGTAAAGGAAGGTATTTTTTGTTTGTTTTAAAAGTACTCAACTTTTATGCACATTAAATTCGTATAATATACTTGG TTAAAGAACATTCAACGTTCAGGCTTTTAGCCTGTTTTCCGTTACATTTGCTGGTCACTAAAGGGAGTAAACTAAAATATGGCCAAC ATGGAACGTCCTCCAATGCTAAAGGATTTTCAACTTCTGAATATATTGTCTACCCTACCCATGGAGTAGGGCAAATTATAGCAATTG AAGATCAAGAAGTTGCAGGACATAAATTAAAGCTTTTCGTCATTCATTTTGCGAAAGATAAAATGGATGTTAAAGTGCCAATTGCAA AAGCCATTTCTGTTGGAATGCGCAAATTATCTGCTGTCGATTCCGTTGAACGTGCATTGAAAATCTTACATGGAAAAGCGCGAGTTA AACGAACCATGTGGTCACGCCGCGCTCAAGAATATGATGCGAAAATTAATTCAGGAGATCTTATTTGTATCGCTGAAGTCGTACGCG ATCTTTTTCGTTCAGATCTACAGCCTGAACAATCTTATTCCGAGCGCAACTCTATATCGCTGCACTTGAAAGGATGGCCCGTGAGA TTGCCGTTATTAATAGTCTTTCTGAAACAGAGGCTATCAATCTCATAGAAATGCATCTCTCCCAAAAGCCAAAGCGTCAATTTAAAA CTAAGAGAGATGAGGTAAATGCAAACAGCGAAGCTGTCTATGCCGCATAATAAAGTGCAAAATCTTTTGATCATAAAATAAAACCAG CGAAATGCTGGTTTCGTTTTTTTATCAAGGCCCTTCAATAATTAGAAGGATATTTGGTCAGAGATTCATCCATTCTTCCTATAAATT CCCCATGCAAATAATATTGGTGTTGAACACAATATAAGTGAAATGAGGTGTGGAGCCTTATCTCTTCACATCCTGTCATAGATGACA TTGATCTTCTACTTCTGCCCTTATGCCTGTCTTTCCAAGAGACTTTTAAGAACACTATGAAGGAAAACATTTCTTTACCGTGCTCTT TCATTGAGAGCCGATAAAAAAAGACAACCTTTACAGTCGCTTCACATAAGCACACTGAAAACAAAGCGCACAGGCAAACGTCACCTG TTAAGATAACAAAAATACAGAAGAAAAACTAAGAGCTTCATCTCCTCTCTGAGAAAGAGAAGAGAAAAACAAAACCTCTGCGCACAT CCCATTCACCGCATAGATTCATTTGTCTCTGAATCAAATGAATCCATCTCTGGTAGAGAGATAAAAATCTCAATCTTTAGCAGGCAA CACTGAACGTCCGCGATACATTCCGGTTTTCAAATCGATGT

Appendix Figure 8.3. - B. henselae carD sequence $+/$ - 600 basepairs The sequence was sourced from the online NCBI genbank. Bold sequence denotes the carD gene. Highlighted sequence denotes primer sites. Blue highlighted sequence denotes sequence removed to form the carD PCR fusion product. 


\section{4. pJS01 Plasmid Confirmation by Sequencing}

All sequencing was performed by Macrogen, South Korea, using universal M13 primers. Sequence readouts were analysed using Geneious bioinformatics software program $8.1 .7^{[233]}$, multiple sequence alignment tool.

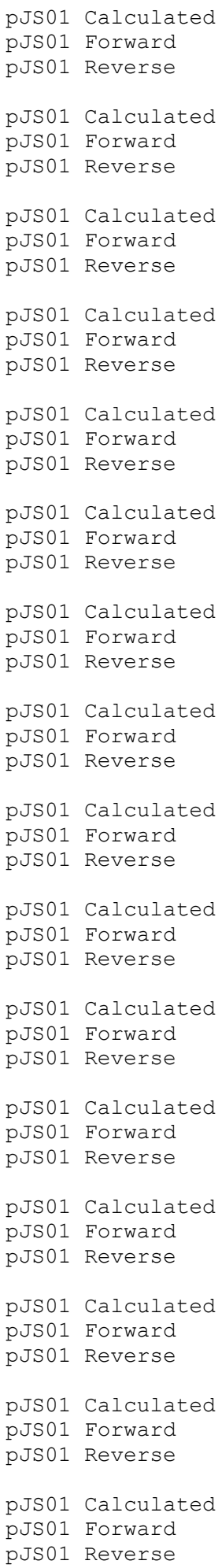

GGCGATTAAGTTGGGTAACGCCAGGGTTTTCCCAGTCACGACGTTGTAAAACGACGGCCA

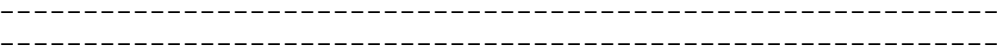
GTGCCAAGCTTGCATGCCTGCAGGTCTCCTGTGAACCAG-AATGCGGATACAGAGCCGGG $----------------------------A G C C A T T A A A C C G G A T A C A G A G C C G G G$

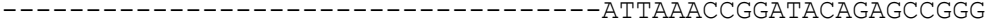

GCTAGAAAAATGGCTAGAGCTCAATCTTCAATATGCAAACAAATGGCCTAATTTAACCAC GCTAGAAAAATGGCTAGAGCTCAATCTTCAATATGCAAACAAATGGCCTAATTTAACCAC GCTAGAAAAATGGCTAGAGCTCAATCTTCAATATGCAAACAAATGGCCTAATTTAACCAC

CAGAAAAGATCCTCTTCCTCAAGCAAAAGAAATGGATGGCATTCCGGATAAATTAGAAAA CAGAAAAGATCCTCTTCCTCAAGCAAAAGAAATGGATGGCATTCCGGATAAATTAGAAAA CAGAAAAGATCCTCTTCCTCAAGCAAAAGAAATGGATGGCATTCCGGATAAATTAGAAAA

GTATTTTTCAGAAAATCCAGGAAGCGGAGAATAATAAGCATAGGACATACATCATAAGCGTATTTTTCAGAAAATCCAGGACGCGGAGAATAATAAGCATAGGACATACATCATAAGCT GTATTTTTCAGAAAATCCAGGACGCGGAGAATAATAAGCATAGGACATACATCATAAGCT

TTTAAAAATTGAAATCATTTCTTTGTAAAAATAAAAATTCCTCAGTGAAATGATTGATTC TTTAAAAATTGAAATCATTTCTTTGTAAAAATAAAAATTCCTCAGTGAAATGATTGATTC TTTAAAAATTGAAATCATTTCTTTGTAAAAATAAAAATTCCTCAGTGAAATGATTGATTC

TTTTGACATTTGATGTTAATGTTATTTAACATGATCATCTTTCTAGGTATTTTTCTTGC TTTTGACATTTGATGTTAATGTTATTTTAACATGATCATCTTTCTAGGTATTTTTCTTGC TTTTGACATTTGATGTTAATTTATTTTAACATGATCATCTTTCTAGGTATTTTTCTTGC

TTTCTCCTTGTAAAGGAAGGTATTTTTTGTTTGTTTTAAAAGTACTCAACTTTTATGCAC TTTCTCCTTGTAAAGGAAGGTATTTCTTGTTTGTTTTAAAAGTACTCAACTTTTGTGCAC TTTCTCCTTGTAAAGGAAGGTATTTCTTGTTTGTTTTAAAAGTACTCAACTTTTGTGCAC

ATTAAATTCGTATAATATACTTGGTTAAAGAACATTCAACGTTCAGGCTTTTAGCCTGTT ATTAAATTCGTATAATATACTTGGTTAAAGAACATTCAACGTTCAGGCTTTTAGCCTGTT ATTAAATTCGTATAATATACTTGGTTAAAGAACATTCAACGTTCAGGCTTTTAGCCTGTT

TTCCGTTACATTTGCTGGTCACTAAAGGGAGTAAACTAAAATATGGCATCCCAACATGGA TTCCGTTACATTTGCTGGTCACTAAAGGGAGTAAACTAAAATATGGCATCCCAACATGGA TTCCGTTACATTTGCTGGTCACTAAAGGGAGTAAACTAAAATATGGCATCCCAACATGGA

GTCCTCAATGCTAAAGGATTTTCAACTTCTGAATATATTGTCTACCCTACCCATGGA

ACGTCCTCCAATGCTAAAGGATTTTCAACTTCTGAATATATTGTCTACCCTACCCATGGA ACGTCCTCCAATGCTAAAGGATTTTCAACTTCTGAATATATTGTCTACCCTACCCATGGA

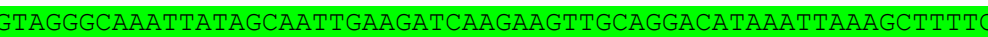
GTAGGGCAAATTATAGCAATTGAAGATCAAGAAGTTGCAGGACATAAATTAAAGCTTTTC GTAGGGCAAATTATAGCAATTGAAGATCAAGAAGTTGCAGGACATAAATTAAAGCTTTTC

GTCATTCATTTTGCGACATCTCTCCCAAAAGCCAAAGCGTCAATTTAAAACTAAGAGAGA GTCATTCATTTTGCGACATCTCTCCCAAAAGCCAAAGCGTCAATTTAAAACTAAGAGAGA

TGAGGTAAATGCAAACAGCGAAGCTGTCTATGCCGCATAATAAAGTGCAAAATCTTTTGA TGAGGTAAATGCAAACAGCGAAGCTGTCTATGCCGCATAATAAAGTGCAAAATCTTTTGA TGAGGTAAATGCAAACAGCGAAGCTGTCTATGCCGCATAATAAAGTGCAAAATCTTTTGA

TCATAAAATAAAACCAGCGAAATGCTGGTTTCGTTTTTTTATCAAGGCCCTTCAATAATT TCATAAAATGAAACCAGCGAAATGCTGGTTTCGTTTTTTTATCAAGGCCCTTCAATAATT TCATAAAATGAAACCAGCGAAATGCTGGTTTCGTTTTTTTATCAAGGCCCTTCAATAATT

AgAAGGATATTTGGTCAGAGATTCATCCATTCTTCCTATAAATTCCCCATGCAAATAATA AGAAGGATATTTGGTCAGAGATTCATCCATTCTTCCTATAAATTCCCCATGCAAATAATA AGAAGGATATTTGGTCAGAGATTCATCCATTCTTCCTATAAATTCCCCATGCAAATAATA 


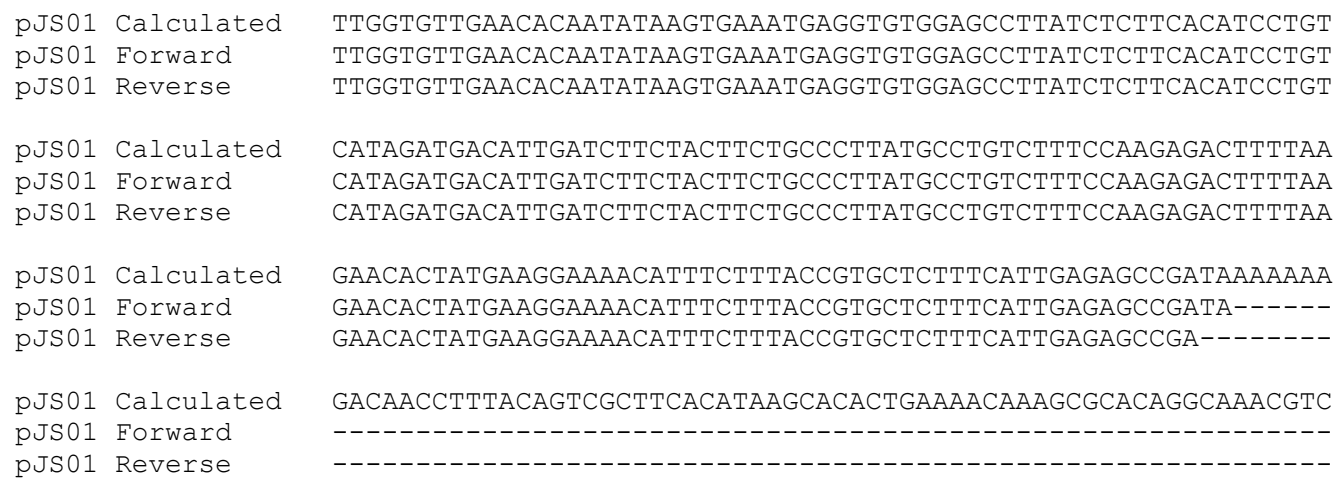

Appendix Figure 8.4. - Macrogen Sequence Alignments using Geneious pJS01 Calculated is an estimate of the pJS01 sequence, constructed from pEX18gm, carD sequences. pJS01 Forward and Reverse sequences are the forward and reverse sequence reads of purified pJS01 plasmids that were sent to Macrogen. Sequence highlighted in green denotes the shortened carD sequence insert. 